\title{
Characterization of ROP GTPase-activated Arabidopsis receptor-like cytoplasmic kinases (RLCK class VI_A)
}

\author{
PhD thesis
}

\author{
Manuela Elena Jurca \\ Supervisor: Dr. Attila Fehér
}

Biological Research Center of the Hungarian Academy of Sciences Institute of Plant Biology

Szeged, 2011 


\section{TABLE OF CONTENTS}

LIST OF ABBREVIATIONS

1. INTRODUCTION

2. SCIENTIFIC BACKGROUND

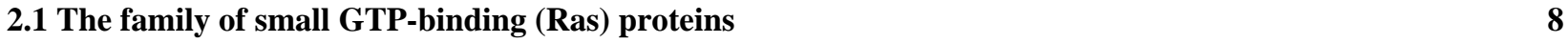

2.2. The Rho family in plants $\quad 9$

2.2.1 Structure of the ROP GTPases $\quad 10$

2.2.2 ROP GTPases: multi-functional signaling switches in plants 13

2.3 Regulators of ROP GTPase Activity $\quad 15$

2.3.1 GDIs - guanine nucleotide dissociation inhibitors 16

$\begin{array}{ll}\text { 2.3.2 GAPs - GTPase activating proteins } & 17\end{array}$

$\begin{array}{ll}\text { 2.3.3 GEFs - nucleotide exchange factors } & 18\end{array}$

2.4 ROP GTPase effectors and their involvement in various signaling pathways 19

2.4.1 ROPs as regulators of the actin cytoskeleton, vesicular trafficking and polarity establishment 21

2.4.2 ROPs as regulators of auxin signaling through ubiquitin/26S proteasome-mediated proteolysis 25

2.4.3 ROPs role in ABA signaling 26

2.4.4 ROPs as regulators of NADPH oxidase and pathogene defence $\quad 26$

2.4.5 ROPs effectors in cell wall synthesis $\quad 29$

2.4.6 ROPs and kinases $\quad 29$

2.5. Plant Receptor-like Kinases

2.5.1 Classification of plant receptor-like kinases (RLKs) 31

2.5.2 Functions of receptor-like cytoplasmic kinase (RLCK) proteins 32

3. AIMS $\quad \mathbf{3 5}$

4. MATERIAL AND METHODS 36

$\begin{array}{ll}\text { 4.1 Sequence analysis } & 36\end{array}$

4.2 Gene expression profiling $\quad 36$

4.2.1 Plant material and growth conditions 36

4.2.2 Nucleic acid isolation $\quad 37$

4.2.3 cDNA synthesis

4.2.4 Real-time quantitative RT-PCR (qRT-PCR) 37

4.2.5. In silico gene expression analysis

4.3. Yeast two-hybrid assay $\quad 39$

$\begin{array}{ll}4.3 .1 \text { Vector construction and mutant generation } & 39\end{array}$

4.3.2 Yeast transformation and selection 41

4.4. Biochemical characterization of AtROPs and AtRLCK VI 42

4.4.1 Expression of AtROPs and AtRLCKs in Escherichia coli 42

4.4.2 In vitro protein interaction $\quad 43$

4.4.3 Protein kinase assay $\quad 44$

4.5. Transgenic plant generation and characterization $\quad \mathbf{4 4}$

4.5.1. Biological material and vector preparation 44

4.5.2 Transformation of Agrobacterium tumefacines $\quad 47$ 
4.5.3 Transformation of Arabidopsis thaliana

4.5.4 Plant selection and molecular characterization

4.5.5 Pollen germination and microscopic visualization

4.5.6 Trichome isolation and morphological analysis

\section{RESULTS}

5.1 Sequence analysis

5.2. Expression analysis

5.2.1 Absolute levels of the RLCKs VI transcripts

5.2.2 Relative expression profile of the 14 RLCK VI genes in Arabidopsis organs

5.2.3 Expression analysis of AtRLCK VI during various treatments

5.2.4 Correlative expression between AtRLCK VI kinases and known/hypothesized ROP regulators and effectors

5.3 Specific interaction of AtRLCKs VI_A kinases with AtROPs in yeast two-hybrid assay

5.7 Characterization of transgenic lines

5.7.1 Selection of transgenic lines over-expressing AtRLCK VI_A2

5.7.2 Selection of transgenic lines silencing AtRLCK VI_A2

5.7.3 Trichome morphogenesis in over-expressing and silencing AtRLCK VI_A2 transgenic plants

5.7.4 Over-expressed and silenced AtRLCK VI_A2 affects pollen tube growth

\section{DISCUSSION AND CONCLUSIONS}

6.2. Gene expression profiling of AtRLCKs VI kinases based on a gene-specific real-time quantitative PCR approach

6.3. Co-expression study of RLCKs and other ROP GTPase effectors and regulators based on in silico data analysis

6.6. Over-expressing and silencing AtRLCK VI_A2 induced modification in trichome branching and pollen tube growth 


\section{LIST OF ABBREVIATIONS}

ABA - abscisic acid

Ade - adenine

ADF - actin depolymerizing factor

ADH - alcohol dehydrogenase

ARF - auxin response factor

ARP2/3 - actin related protein $2 / 3$

At - Arabidopsis thaliana

ATP - adenosine triphosphate

Aux/IAA proteins - auxin/indole acetic acid-inducible proteins

CA - constitutive active form

cDNA - complementary DNA

Cdc42 - cell division cycle 42, Rho GTPase

CDPK- calcium dependent protein kinases

CRIB- CDC42/RAC-interactive binding motif

DH - Dbl homology domain

DHR - DOCK homology domain

DN - dominant negative form

DRF - diaphanous- related formins

DRMs - detergent -resistant membranes

DUF - domain of unknown function

EOX - estradiol inducible over-expressing lines

ERNAi - estradiol inducible RNAi lines

FH - formin-homology region

GAP - GTPase activating protein

GDI - guanine nucleotide dissociation inhibitor

GDP - guanidine diphosphate

GEF - guanine nucleotide exchange factor

GPCR - G-protein coupled receptor

GTP - guanidine triphosphate

GTPase - guanine triphosphatase

His - histidine

HOX - heat inducible over-expressing lines

ICR1 - interactor of constitutively active ROP

IPTG - isopropyl-1- thio-/3-Dgalactopyranoside

kDa - kilodaltons

L- non-prenylated, localization mutant

Leu - leucine

MAPK-mitogen activated protein kinase

MBP - maltose binding protein

MPK1 - mitogen-activated protein kinase 1

mRNA - messenger RNA

Ms - Medicago sativa

Mt - Medicago truncatula

MT - microtubules

MyBP - myelin basic protein

NAK - novel Arabidopsis protein kinase

NCRK - cysteine-rich receptor kinase

NOX - NADPH oxidase 
Nt - Nicotiana tabacum

ORF - open reading frame

Os - Oryza sativa

PAK1 - p21-activated kinase 1

PH - pleckstrin homology domain

PM - plasma membrane

PRONE - plant specific ROP nucleotide exchanger

PtdInsP-K - phosphatidylinositol monophosphate

Pti1 - Pto-interacting 1 protein

RB - retinoblastoma protein

RBOH - respiratory burst oxidase homolog

$\operatorname{Rep} A(\mathrm{C} 1)$ - protein of subgroup I geminiviruses

RIC - ROP-interactive CRIB-containing protein

RLCK - receptor-like cytoplasmic kinase

RLK - receptor-like kinase

ROP - Rho protein of Plant

ROS - reactive oxygen species

RRK- ROP-interacting receptor-like kinase

RTK - receptor tyrosine kinase

RT-PCR - reverse transcription polymerase chain reaction

qRT-PCR - real-time quantitative PCR

SCAR - suppressor of cyclic AMP receptor

SDS-PAGE - sodium dodecyl sulphate-polyacrylamide gel electrophoresis

SEC3 - subunit of exocyst 3

Ser- serine

SPK1/SPIKE1 -DOCK family guanine nucleotide exchange factor (GEF)

T-DNA - transferred-DNA

Thr- threonine

Trp - tryptophan

Tyr- tyrosine

WASP - Wiskott-Aldrich syndrome proteins (WASP)

WAVE - WASP family verprolin-homologous protein

WT - wild type

Y2H - yeast two hybrid

Z. elegans - Zinnia elegans

Zm - Zea mays

One-letter code amino acid:

$\begin{array}{llll}\text { Alanine } & \text { A } & \text { Leucine } & \text { L } \\ \text { Arginine } & \text { R } & \text { Lysine } & \text { K } \\ \text { Asparagine } & \text { N } & \text { Methionine } & \text { M } \\ \text { Aspartic acid } & \text { D } & \text { Phenylalanine } & \text { F } \\ \text { Cysteine } & \text { C } & \text { Proline } & \text { P } \\ \text { Glutamine } & \text { Q } & \text { Serine } & \text { S } \\ \text { Glutamate } & \text { E } & \text { Threonine } & \text { T } \\ \text { Glycine } & \text { G } & \text { Tryptophan } & \text { W } \\ \text { Histidine } & \text { H } & \text { Tyrosine } & \text { Y } \\ \text { Isoleucine } & \text { I } & \text { Valine } & \text { V }\end{array}$




\section{INTRODUCTION}

Higher plants, being rooted, are bombarded by a wealth of external stimuli. Their cells are also subject to many endogenous signals, such as hormones and developmental cues, some of which comes from neighboring cells and involve intercellular communication. All these signals must be recognized, integrated, and translated into responses at the cell or organ level. The mechanisms of signal perception and many components of signaling pathways are highly conserved among eukaryotes, although plants have evolved along different lines from animals and show some unique features and novel combinations of ancient themes.

Ample research on experimental models such as Drosophila, Caenorhabdites, Xenopus oocytes, mammalian cell lines, and yeast, lead to identification of basics elements involved in signaling that are remarkably conserved. An extracellular signal binds to or stimulates a plasma membrane-based receptor, which activates a GTP-binding protein (G-protein). The G-protein alternates between two conformational states GDP (inactive form) or GTP (active form) bound states and thus acts as a molecular switch. GTP is hydrolyzed to GDP by an intrinsic GTPase activity in the protein. When activated, the G-protein either regulates a cascade of protein kinases or modulates the activity of effector molecules and second messengers, which, in turn, regulate the activities of protein kinases. Protein kinases regulate the activities of other proteins and/or enzymes, which mediate gene expression or metabolic pathways. Protein phosphatases are also recognized as important components of signaling. The signal perception/transduction pathways, thus, involve at least four elements: receptors, G-proteins, effector molecules and second messengers, and protein kinases and phosphatases. Two classes of signaling $G$ proteins are known: heterotrimeric G proteins and monomeric RAS superfamily of small GTPases.

In plants, the intracellular signaling pathways linking cell surface receptors to nuclear or metabolic responses remain poorly understood. The Arabidopsis genome sequence reveals that plants lack many of the signaling G proteins used by animals and yeast. Only one $\alpha$-, one $\beta$ - and two $\gamma$ - subunits of the heterotrimeric G-protein have been identified (GPA1, AGB1 and AGG1, AGG2) in the Arabidopsis genome and no G-protein coupled receptor (GPCR) has been unequivocally identified in plants (Jones 2002; Jones and Assmann 2004; Johnston et al. 2007).

Plants, however, contain a unique family of small GTPases, termed ROP (Rho-of-plants). Rho-type GTPases belong to the RAS superfamily of small GTP-binding proteins which serve as two-state molecular switches depending on their GDP- or GTP-bound conformation (Wennerberg 
et al. 2005; Lundquist 2006). Rho GTPases are implicated in diverse cellular processes through the regulation of cytoskeletal organization and dynamics, NADPH oxidase activity and gene expression (Bokoch 2000; Bustelo et al. 2007). The GTP-binding and hydrolyzing activity of these multifunctional proteins is tightly regulated by a dedicated group of protein factors (Narumiya 1996). The effector proteins downstream of Rho GTPases are also numerous and further increase the specificity of Rho GTPase signaling (Cotteret and Chernoff 2002; Karnoub et al. 2004). Rho-type small GTPases are ancient proteins present in most eukaryotes with considerable structural conservation. However, the early split of viridiplantae form the animalfungal-amobeozoa lineage led to the separated evolution of plant ROP (as Rho-of-plants) GTPases (Brembu et al. 2006). This separation resulted in the accumulation of unique features as the primary structure, regulation and signaling properties of ROP GTPases are considered (Brembu et al. 2006; Berken and Wittinghofer 2008). Among others, no Cdc42/Rac-interactivebinding (CRIB) motif-containing kinases (p21-activated kinases or PAKs), characteristic for other eukaryotes (Hofmann et al. 2004; Cotteret and Chernoff 2002), could be identified in plants. PAKs play key roles in fundamental and general cellular processes, such as cytoskeletal rearrangements and the stimulation of mitogen-activated protein kinase (MAPK) cascades, in animal and yeast cells (Hofmann et al. 2004; Bokoch 2003). Therefore it is striking that similar pathways have not been identified in plants up to now. Moreover, plants do not have cognate RasGTPases although these proteins are also key elements of MAPK-mediated mitogen signaling in other eukaryotes (Dunn et al. 2005). It is generally believed therefore that ROP GTPases, as the only signaling type small GTPases in plants with combined Rho and Ras functions, should be linked to kinase cascades in a presently unknown way (Berken 2006; Yang and Fu 2007). In our laboratory a previous research showed that ROPs interact with and activates RLCKs in Medicago (Dorjgotov et al. 2009), and the aim of this work was to characterize the function of corresponding RLCK group in Arabidopsis. 


\section{SCIENTIFIC BACKGROUND}

\subsection{The family of small GTP-binding (Ras) proteins}

Rho-type small guanine nucleotide binding proteins together with Ras, Ran, Arf and Rab proteins compose the RAS superfamily with evolutionary conserved orthologues in most eukaryotic organisms (Colicelli 2004; Takai et al. 2001; Wennerberg et al. 2005).

These small G proteins cycle between their active GTP-bound and the inactive GDPbound conformation as they can slowly hydrolyze GTP (Vetter and Wittinghofer 2001). Based on the latter activity, the proteins are also known as GTPase, although they are poor catalysts. Therefore their GTPase cycle is facilitated by other proteins: GDP/GTP exchange is catalyzed by guanyl nucleotide exchange factors (GEFs), and the GTP hydrolysis is enhanced by GTPase activating proteins (GAPs).

Most members of the Ras superfamily of small G-proteins, associate with and function at cell membranes because of their posttranslational lipid modifications, prenylation and S-acylation (Hancock et al. 1989; Michaelson et al. 2001). Guanyl nucleotide dissociation inhibitors (GDIs) inhibit GDP/GTP exchange and facilitate the cycling of these GTPases on and off membranes (DerMardirossian and Bokoch 2005).

Only the active GTP-form is able to interact with downstream effectors to exert its biological function. Ran, Arf and Rab proteins control nuclear and vesicular trafficking processes in eukaryotic cells. Rho and Ras family members regulate extracellular stimulus-dependent signaling pathways that affect gene expression, cell proliferation, differentiation and survival in the case of Ras, and actin reorganization, cell cycle progression, cell polarity and gene expression in case of the Rho proteins (Wennenberger et al. 2005; Lundquist 2006).

The Arabidopsis genome sequence reveals that plants lack several signaling small $\mathrm{G}$ proteins used by yeast and metazoa (Vernoud et al. 2003). More interestingly, cognate Ras proteins have not been identified in plants which leaves the Rho family into the center of attention for small $\mathrm{G}$ protein regulated signaling events in these organisms (Berken 2006).

In animals and fungi, the Rho family itself is further divided into subfamilies of Rho, Rac, and Cdc42 proteins, which are not found in plants either (Boureux et al. 2007; Vernoud et al. 2003; Zheng and Yang 2000). Instead, plants have a unique class of Rho proteins, which, according to today's predominant nomenclature, is mostly referred to as ROPs (for "Rho of 
plants") or RACs (based on the sequence similarity they share with animal RAC proteins forming a Rho subfamily) (Winge et al. 2000; Christensen et al. 2003).

\subsection{The Rho family in plants}

In every plant species that has been examined, ROPs belong to a multigene family that was formed by gene duplications during evolution (Winge et al. 2000). Several genes encoding ROP proteins have already been isolated from species such as Arabidopsis (Li et al. 1998), barley (Schultheiss et al. 2003), rapeseed (Chan and Pauls 2007), cotton (Delmer et al. 1995; Kim and Triplett 2004), maize (Hassanain et al. 2000), tobacco (Morel et al. 2004), alfalfa (Szücs et al. 2006, Dorjgotov et al. 2009), and rice (Christensen et al. 2003).

The subgroup of plant Rho-like GTPases (ROP) has been subjected to intense research since 1993 when the first plant cDNA encoding a Rho-related GTPase (Rho1Ps) from pea was cloned (Gu et al. 2004; Yang 2002; Zheng and Yang 2000). The most characterized proteins are the eleven ROPs from Arabidopsis (AtROP1 to AtROP11) (Li et al. 1998; Winge et al. 2000). Sequence analysis revealed that they share $70-98 \%$ amino acid identity with each other and at least $45 \%$ identity with other members of the Rho family. It was shown that the Arabidopsis ROPs are more similar to the mammalian Rac proteins with up to $61 \%$ identical residues with human Rac1 (Winge et al. 2000; Zheng and Yang 2000). Therefore, the ROPs have also been denoted as plant RAC proteins, and based on a recent evolutionary analysis of the Rho family in eukaryotes, the Rac genes are probably the founders of the ROP but also of the Cdc42 subfamily (Boureux et al.2007). Moreover, this study clearly differentiates between the clusters of the ROP, Rac, Cdc42 and Rho proteins underlying the opinion that ROPs represent a unique Rho clade that is specific for plants (Agrawal et al. 2003; Zheng and Yang 2000).

ROPs cover a vast spectrum of Rho functions in plant cells and thus structurally diverged to allow interactions with diverse cellular targets. Concerning the classification of ROP proteins, two methods are described in the literature. The first classification, that placed ROP GTPase as a branch in the Rac subfamily, divide them into two subgroups (type I and II), according to the structure of their C-terminal hypervariable domain (Winge et al. 1997). The second classification method, which is based on nucleotide sequences, suggested that ROP GTPases diverged as a separate group prior to the separation between Rac and $\mathrm{Cdc} 42$ and can be divided into four subgroups, which were designated I, II, III, and IV. Subgroups I and II correspond to type II ROP GTPases, and subgroups III and IV correspond to type I ROP GTPases of the first classification 
method (Yang 2002; Christensen et al. 2003; Vernoud et al. 2003). A more recent study, based on the sequence analysis of the whole Rho family in different eukaryotes confirmed that actually both earlier analyses were correct (Boureux et al. 2007).

ROP GTPases are indeed more closely related to Rac, but they diverged as a separate Rac group prior to the divergence of Rac, Rho and Cdc42. The unique features discovered in high resolution three-dimensional structures of AtROP9/RAC7 (Sormo et al. 2006) and AtROP4/RAC5 (Thomas et al. 2007) support the notion that ROP GTPases form a unique subgroup of the Rho GTPases (Berken and Wittinghofer 2008).

\subsubsection{Structure of the ROP GTPases}

Ras-type small guanine nucleotide-binding proteins act as molecular switches cycling between their active, GTP-bound, and inactive, GDP-bound conformations. Their structure consists of a central six-stranded mixed $\beta$-sheet surrounded by $\alpha$-helices and loops (Figure 1).

Analysis of the sequences and structures of Ras and related proteins identified highly conserved motifs associated with GTPase activity (Kaziro et al. 1991). These motifs can be found in plant ROP GTPases as well and include the $\mathrm{G}$ domain, which facilitates nucleotide binding and hydrolysis through the interaction with GTPase regulatory proteins, and a hypervariable domain that determines subcellular targeting (Bourne et al. 1991; Sprang 1997; Takai et al. 2001; Leipe et al. 2002; Wennerberg et al. 2005; Vetter and Wittinghofer 2001).

The G-domain contains five highly conserved sequence motifs (G-box motifs, G1-G5). In plant proteins the consensus sequences for those G-box-motifs (Figure 1) have been established by comparison of multiple monocot and dicot ROP proteins (Christensen et al. 2003), variations represented are in good agreement with the consensus motifs originally described by Bourne and colleagues (Bourne et al. 1991). In order to obtain constitutively active (CA) or dominant negative (DN) ROP forms, key residues within the G-box-motifs are mutated (Figure 1A). It is believed that substitutions of an invariant glycine in G1 (G15 in ROP9) or the glutamine in G3 (Q64 in ROP9) interfere with GTP hydrolysis thus keeping the ROPs in the active state and mutating the consensus threonine in G1 (T20 in ROP9) or the aspartate in G4 (D121 in ROP9) is thought to result in reduced nucleotide and increased GEF affinities (Yang 2002).

The three-dimensional structure information on the Arabidopsis type-II AtROP9 in its free form bound to GDP (PDB code 2J0V (Sormo et al. 2006)) and type-I AtROP4 in complex with GDP and the catalytic domain PRONE (plant-specific ROP nucleotide exchanger) of its 
activating GEF (PDB code 2NTY (Thomas et al. 2007)) showed the G protein with the typical topology (Paduch et al. 2001; Vetter and Wittinghofer 2001; Wennerberg et al. 2005). It folds into a globular molecule composed of a central $\beta$-sheet with 6 strands $(\beta 1-\beta 6)$ that are surrounded on both sides by a-helices as it was expected (Figure 1B).

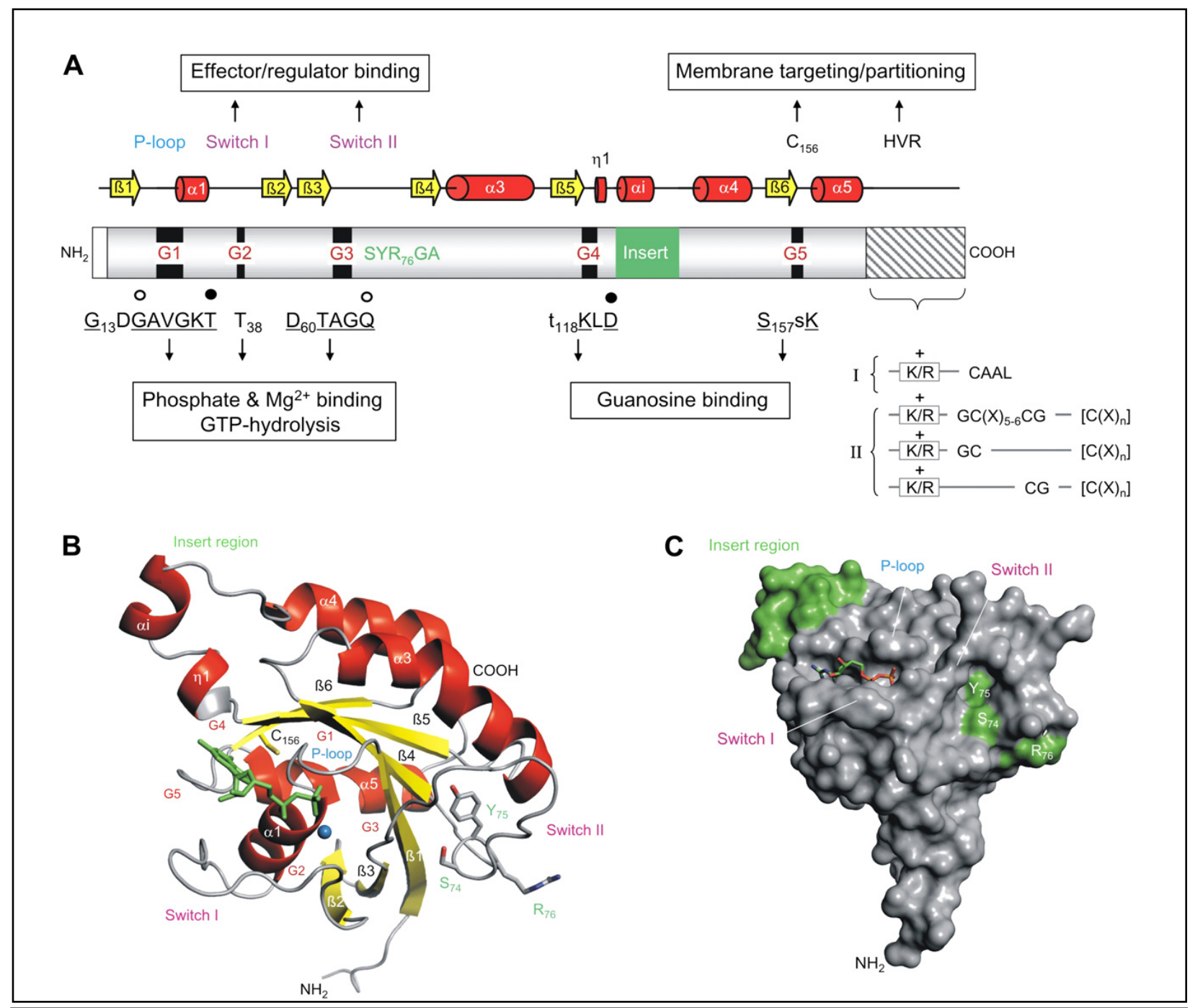

Figure 1: (A) Structural features of ROP proteins and their assigned function (Berken and Wittinghofer 2008). G domain (grey), Rho insert (green) and C-terminal hypervariable region (HVR: stripy); G-box-motifs (G1-G5) with their consensus sequence below; sites of the P-loop, the switch regions, the ROP-specific motif 'SYRGA' downstream of G3 in switch II and a putatively acylated cysteine (C156) within the G domain are indicated. Distinct HVR structures of type-I (I) and type-II (II) ROPs are given schematically (Lavy and Yalovsky 2006), including the polybasic region (K/Rbox: multiple positively charged $(+) \mathrm{K}$ and $\mathrm{R}$ residues) and variations of the consensus motifs GC-CG and CAAL. Secondary elements (a-helices: red cylinders; b-strands: yellow arrows; loops: black lines) are presented on the top based on the tertiary structure of ROP9 from Arabidopsis (Sormo et al. 2006). (B) 3D-structure of AtROP9 bound to GDP (stick representation in green) and $\mathrm{Mg}^{2+}$ (blue sphere) (2J0V (Sormo et al. 2006)). (C) Surface representation of AtROP9 bound to GDP (90_flipped view of B) highlighting the exposed insert region and the putative kinase recognition signature (SYR) within the 'SYRGA'-motif. Positions of the P-loop and the switch regions are indicated for orientation. Models in (B) and (C) were prepared using the program PyMOL (http://www.pymol.org). 
Recently, study on the structure of a ternary complex of GDP-bound AtROP4 with the catalytic domain of its regulatory GEF shows a vigorous interaction of the GEF with the switch regions (Thomas et al. 2007).

In addition to the $\mathrm{G}$ domain, the ROPs display a $\mathrm{C}$-terminal variable region known as hypervariable regions (HVR) that can be posttranslationally modified with lipid moieties to target the proteins to the plasma membrane (PM) where their activation occurs (Wennerberg and Der 2004). Through binding to GDIs (guanine nucleotide dissociation inhibitors) the ROPs recycled back to the cytosol or to other membranes (DerMardirossian and Bokoch 2005; Dransart et al. 2005).

Type-I ROPs usually display a CAAL-box Cys at the extreme C-terminus, which is prenylated in cytoplasm by geranylgeranyltransferase I (PGGT; Sorek et al. 2007). The cysteine in this motif is believed to be modified by isoprenoid chains (Lavy et al. 2002; Lavy and Yalovsky 2006). Following prenylation, proteins undergo two additional posttranslational modifications, collectively referred to as CaaX processing. The first of these modifications involves proteolytic removal of the last three amino acids by either of two CaaX proteases called Ste24 and Rce1. In turn, the free carboxyl group of the isoprenyl Cys is methylated by isoprenyl carboxymethyltransferase (Young et al. 2000).

The hypervariable domain of type-II ROPs consist of a unique sequence motif designated the GCCG box and a proximal plybasic domain. The S-acylation of two or three $\mathrm{C}$ terminal cysteines in the HVR have been reported (Lavy et al. 2002; Lavy and Yalovsky 2006), and variations of a conserved so called GCCG box have been identified, in which the modified cysteines are flanked by glycine residues (Lavy and Yalovsky 2006) (Figure 1A).

Recently it was reported that the transient S-acylation ROPs also occurs on a conserved cysteine within the G domain (C156 in ROP9, Figure 1A), and this cysteine is conserved in nonplant Rho proteins as well (Sorek et al. 2007). This kind of acylation in type-I ROPs apparently goes along with their activation and partitioning in detergent-resistant membranes (DRMs) known as lipid rafts (Sorek et al. 2007).

The "Rho insert region" is an additional feature of the ROPs which allowed their characterization as bona fide Rho proteins. It is an insertion of 9-11 amino acids between $\beta$-strand 5 and $\alpha$-helix 4 of the $\mathrm{G}$ domain. The Rho insert, too, forms a helical structure known as insert helix ( $\alpha \mathrm{i})$ that is followed by a short loop. These elements stick out of the conserved $\mathrm{G}$ domain 
making the insert accessible to the surrounding medium (Sormo et al. 2006) (Figures 1 B, C). Concerning the role of "Rho insert region", in animals and fungi it has been discussed that it may have a role in recognition and activation of specific effectors molecules (Freeman et al. 1996; Karnoub et al. 2001; Thapar et al. 2002; Walker et al. 2000). In plants, recent data showed that is involved in the interaction of the ROPs with their regulatory GEFs (Thomas et al. 2007). Moreover, we showed that the "Rho insert region" of ROP is required for the activation of a RLCK VI kinase (RRK1) in Medicago (Dorjgotov et al. 2009).

\subsubsection{ROP GTPases: multi-functional signaling switches in plants}

The activity of the ROP switch governs a variety of processes in the life cycle of a plant like cell polarity, growth and morphogenesis, cytokinesis, hormone signaling and plant-microbe interactions (Brembu et al. 2006; Berken 2006; Gu et al. 2004; Nibau et al. 2006; Yang and Fu 2007). Moreover, it was found that ROPs are involved in endo- and exocytotic processes and membrane recycling (Bloch et al. 2005; Lavy et al. 2007), abiotic stress responses (BaxterBurrell et al. 2002; Xin et al. 2005), meristem maintenance (Lavy et al. 2007; Trotochaud et al. 1999), xylogenesis (Ko et al. 2006; Brembu et al. 2005), and microspore development (Chan and Pauls 2007) as well. ROP GTPases are implicated in these processes through the regulation of cytoskeletal organization and dynamics, NADPH oxidase activity and gene expression (Bokoch 2000; Bustelo et al. 2007) (see Figure 2). This wide range of biological functions is largely dependent on the interaction of ROPs with a variety of upstream regulators and downstream effector proteins. 


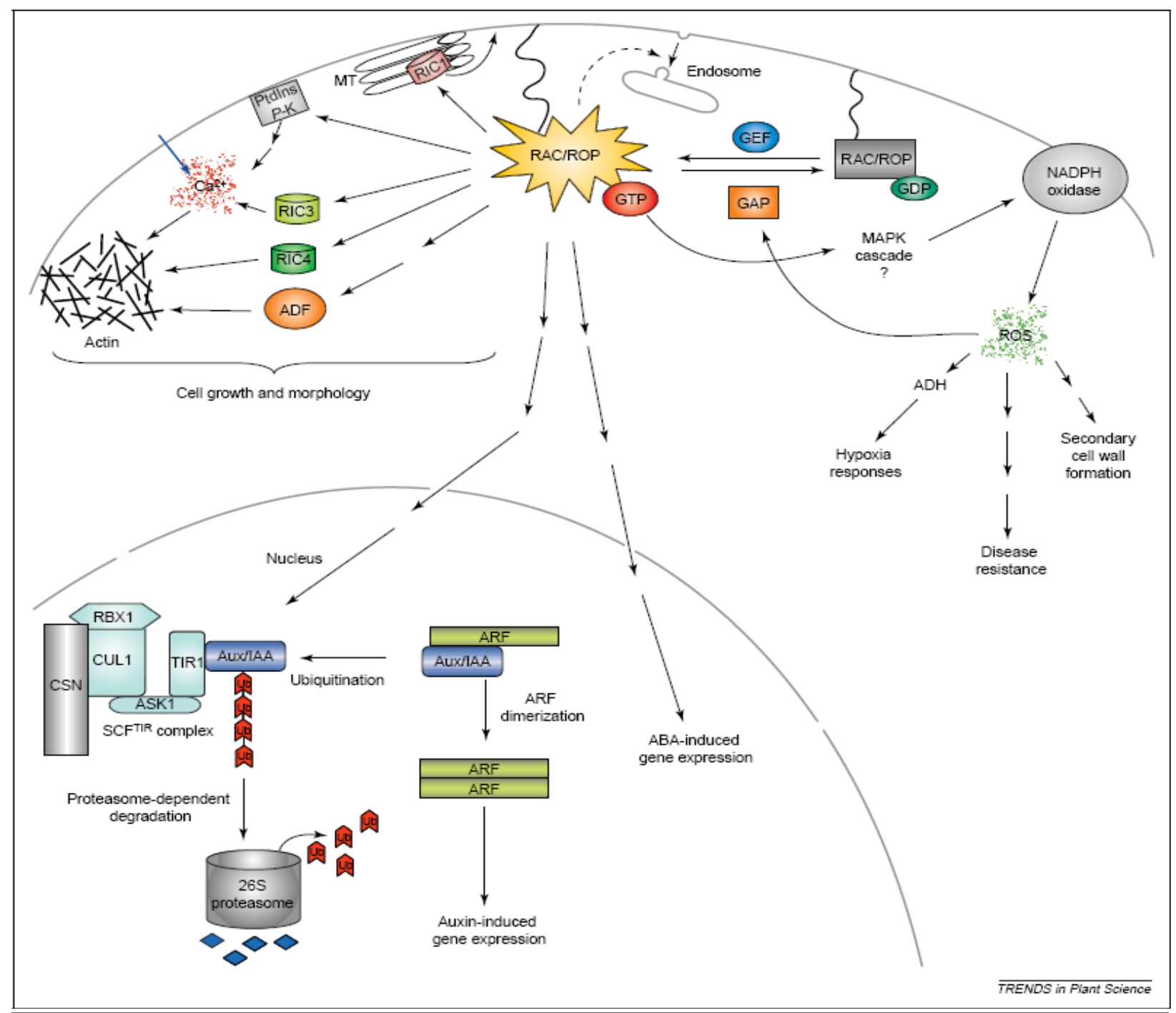

Figure 2: A schematic representation of divergent ROP-regulated signaling pathways (from Nibau et al. 2006). Major targets for ROP GTPases include cell growth and morphology, gene expression and reactive oxygen species (ROS) generation. ROPs regulate actin polymerization, microtubule bundling, Ca2+ fluxes during polar growth, cell expansion and morphogenesis through the activation of RICs (ROP-interactive CRIB motif-containing proteins). ROP regulation of PtdInsP-K (phosphatidylinositol monophosphate kinase) affects phosphatidylinositol (4,5)-bisphosphate signaling, which, in turn, impacts the actin cytoskeleton through regulating the activity of actinbinding proteins and calcium homeostasis, which also affects actin dynamics. ROP regulate ROS production, most probably through the activation of a membrane-bound NADPH oxidase and a MAP kinase cascade might mediate this activation. ROS regulate alcohol dehydrogenase (ADH) and control responses to oxygen deprivation. In regulating the hypoxia response, ROS stimulate GAP expression, providing a negative feedback loop to regulate RAC/ROP activity, presumably to ensure a sub-toxic level of ROS production. ROP regulate auxin and ABA signaling. ROPs are activated by auxin and mediate auxin-induced gene expression by targeting degradation of Aux/IAA proteins, through the ubiquitin/proteasome-dependent proteolysis pathway. Disruption of Aux/IAA and auxin response factor (ARF) interaction leads to auxin-induced gene expression. CSN indicates Cop9 signalosome, which is known to interact with the auxin-signaling pathway. ROPs are inactivated by ABA, whereby several ABA-regulated responses are induced, including gene expression. The intervening signaling components involved in these ROP-regulated pathways remain to be identified. 


\subsection{Regulators of ROP GTPase Activity}

The intrinsic GTPase activity of Rho GTPases hydrolyzes GTP to GDP, leading to conformational changes that abolish interactions with downstream effectors. The switch back to the GTP-bound state occurs when GDP is released and replaced by GTP.

The GTPase cycle of plant Rho GTPases is facilitated by three types of proteins: the nucleotide exchange factors (GEFs), the GTPase activating proteins (GAPs) and the guanine nucleotide dissociation inhibitors (GDIs) (Geyer and Wittinghofer 1997) (Figure 3). These regulatory proteins are the products of small gene families in Arabidopsis (Brembu et al. 2006; Berken and Wittinghofer 2008, Table 1).

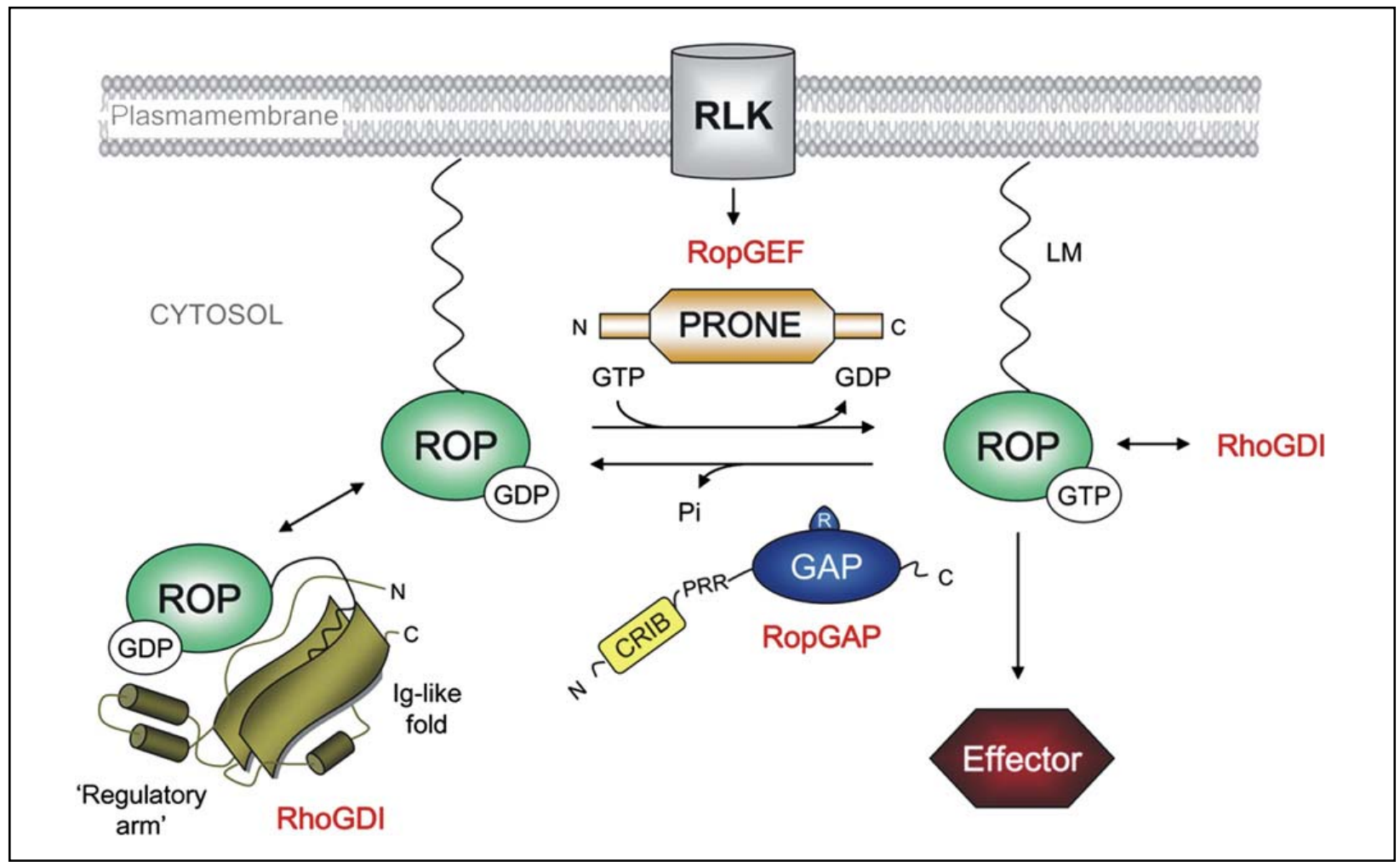

Figure 3: Schematic illustration of functionally relevant domains and motifs in the regulators of the ROP cycle (from Berken and Wittinghofer 2008). Membrane attached ROPs are activated by RopGEFs via the catalytic PRONE domain promoting GDP/GTP exchange. The GTPase activity of ROPs is stimulated by RopGAPs requiring both a CRIB-motif and a GAP domain with an arginine finger (R) for efficient catalysis (PRR: proline-rich region of RopGAPs with unknown function). RhoGDIs sequester ROPs in the cytosol by hiding the C-terminal lipid-moieties (LM) within their immunoglobulin (Ig)-fold while a regulatory arm binds to ROPs preventing GDP dissociation or GTP hydrolysis. Upstream signals can feed into this regulatory cycle via a putative contact between receptor-like kinases (RLK) and RopGEFs. Downstream pathways are induced through the interaction of GTP-bound ROP with effectors. 


\subsubsection{GDIs - guanine nucleotide dissociation inhibitors}

In the Arabidopsis genome three genes encoding ROP GDI-like proteins can be found (Berken and Wittinghofer 2008) (Table 1). The approximately $22 \mathrm{kDa}$ proteins contain highly conserved amino acids in their isoprene binding pocket and exhibits $29 \%$ to $37 \%$ similarity to known mammalian homologues (Bischoff et al. 2000).

Table 1: ROP regulators and their experimentally indicated interactions with ROPs (only Arabidopsis genes are given, even if the interaction of homologous proteins was detected in other species) (Feher et al. 2008)

\begin{tabular}{|c|c|c|c|c|c|}
\hline Annotation & Name & AGI & Affy ID & Interaction & References \\
\hline \multirow{6}{*}{$\begin{array}{c}\text { ROP GTPase } \\
\text { activating protein }\end{array}$} & GAP1 & At4g03100 & 255410_at & AtROP1 & Wu et al. 2000 \\
\hline & GAP2 & At5g22400 & 249933_at & AtROP1 & Wu et al. 2000 \\
\hline & GAP3 & At2g46710 & 266324_at & AtROP1 & Wu et al. 2000 \\
\hline & GAP4 & At3g11490 & 259287_at & AtROP2 & $\begin{array}{l}\text { Baxter-Burrell et al. } \\
2002\end{array}$ \\
\hline & GAP5 & At1g08340 & 261809_at & & \\
\hline & GAP6 & At2g27440 & 265666_at & & \\
\hline \multirow{3}{*}{$\begin{array}{l}\text { ROP GDP-dissociation } \\
\text { inhibitor protein }\end{array}$} & GDI1 & At3g07880 & 258637_at & AtROP4 & Carol et al. 2005 \\
\hline & GDI2a & At1g62450 & 265115_at & NtRAC5 & Klahre et al. 2006 \\
\hline & GDI2b & At1g12070 & 264395_at & & \\
\hline \multirow{15}{*}{$\begin{array}{l}\text { ROP guanyl- } \\
\text { nucleotide exchange factor }\end{array}$} & GEF1 & At4g38430 & 252975_s_at & AtROP1, 4 & $\begin{array}{l}\text { Berker et al. } 2005 \text {; } \\
\text { Gu et al. } 2006\end{array}$ \\
\hline & GEF2 & At1g01700 & 261590_at & AtROP4 & \\
\hline & GEF3 & At4g00460 & 255757_at & & \\
\hline & GEF4 & At2g45890 & 266913_at & & \\
\hline & GEF5 & At5g05940 & 250756_at & & \\
\hline & GEF6 & At3g55660 & 251778_at & & \\
\hline & GEF7 & At5g02010 & 251080_at & & \\
\hline & GEF8 & At3g24620 & 258129_at & AtROP4 & Thomas et al. 2007 \\
\hline & GEF9 & At4g13240 & 254757 at & AtROP1 & Gu et al. 2006 \\
\hline & GEF10 & At5g19560 & 245945_at & & \\
\hline & GEF11 & At1g52240 & 259836_at & & \\
\hline & GEF12 & At1g79860 & 260161_at & & \\
\hline & GEF13 & At3g16130 & 258330_at & & \\
\hline & GEF14 & At1g31650 & 246576_at & AtROP1 & Gu et al. 2006 \\
\hline & SPIKE1 & At4g16340 & 245492_at & $\begin{array}{l}\text { AtROP2, 3, 4, 5, } \\
8,10,11\end{array}$ & $\begin{array}{l}\text { Uhrig et al. 2007; } \\
\text { Basu et al. } 2008\end{array}$ \\
\hline
\end{tabular}


Most Rho GTPases are post-translationally modified by isoprenylation of the cysteine residue within a C-terminal CAAX box (Seabra 1998). This lipid modification is responsible for the association of Rho GTPases with appropriate membrane regions where they exert their function (Michaelson et al. 2001). RhoGDI proteins interact with and are able to remove prenylated Rho GTPases from membranes and form cytoplasmic heterodimers with them (Michaelson, et al. 2001). In this way, they negatively regulate the signaling activity of Rho GTPases (Figure 3).

Using the yeast two-hybrid system and in vitro assays, it could be demonstrated that AtRhoGDI1 specifically interacts with both AtROP4 and AtROP6 (Bischoff et al. 2000). AtRhoGDI1 expression was found to be ubiquitous in the Arabidopsis organs (Bischoff et al. 2000). Co-expression of the AtRhoGDI1 gene with the NtRAC1 gene in transfected tobacco protoplasts repressed NtRAC1-dependent auxin-responsive promoter activation, indicating that these proteins indeed function as negative regulators of ROP GTPase signaling in planta (Michaelson et al. 2001). Altered ("more cytoplasmic") intracellular localization of the GTPase if co-expressed with the GDI gene was also observed (Tao et al. 2002). AtRhoGDI1 was also implicated in the spatial regulation of root hair growth and was hypothesized to control the activity of the NADPH-oxidase (RHD2/AtrbohC) complex in this context (Carol et al. 2005). Nicotiana tabacum L. Nt-RhoGDI2 has been shown to be highly and specifically expressed in elongating pollen tubes where it regulates the polar localization of the Nt-RAC5 GTPase and pollen tube expansion (Klahre et al. 2006). Although Nt-RhoGDI1 has also been shown to interact with Nt-RAC5, it is not expressed in flower or pollen where Nt-RAC5 is expressed at the highest level, but both proteins are expressed in the shoot apex and young leaves where they may have functional interaction (Kieffer et al. 2000).

\subsubsection{GAPs - GTPase activating proteins}

GAP proteins accelerate the hydrolysis of Rho-bound GTP and therefore promote the inactivation of Rho GTPases (Peck et al. 2002). The reaction mechanism frequently involves an arginine residue that resides in the catalytically active GAP domain of the protein. This so-called arginine finger is inserted into the active site of the protein to stabilize the transition state during GTP hydrolysis (Scheffzek and Ahmadian 2005) (Figure 3).

The Arabidopsis genome encodes six GAP proteins (Table 1). All Arabidopsis GAPs contain the conserved CRIB (CDC42/RAC-interactive binding) motif playing a role in binding 
the GTPase (Wu et al. 2000). In yeast and animals, CRIB domains mediate GTP-dependent interaction between Rho GTPases and their effector proteins, but are not found in conjunction with RhoGAP domains. Point and deletion mutants in the CRIB region reduced the intrinsic ROPGAP activity in vitro, documenting a critical role of this protein interaction motif in plant GAP activity ( $\mathrm{Wu}$ et al. 2000). In tobacco pollen tubes, Nt-RhoGAP1 has been shown to be localized subapically and hypothesized to restrict Nt-RAC5 activity to the apical region (Klahre and Kost 2006) in concert with Nt-RhoGDI2 action (Kost 2008). A negative feedback loop including At-ROPGAP4 and AtROP2 was revealed in Arabidopsis seedlings subjected to oxygen deprivation. It was shown that ROPGAP4 transcription is increased due to the active At-ROP2dependent production of $\mathrm{H}_{2} \mathrm{O}_{2}$ (Baxter-Burrell et al. 2002).

\subsubsection{GEFs - nucleotide exchange factors}

Guanine nucleotide exchange factors (GEFs) catalyze the dissociation of GDP from the inactive Rho proteins and allow the binding of GTP, which induces a conformational change that permits interaction with downstream effectors (Geyer and Wittinghofer 1997; Schiller 2006). Due to this activity, GEFs are primarily responsible for linking cell-surface receptors to Rho protein activation in metazoa (Schiller 2006) (Figure 3).

In animals, two families of RhoGEFs have been identified. The members of Dbl family of RhoGEFs contain a Dbl homology (DH) domain followed by a PH (pleckstrin homology) domain (Rossman et al. 2005). The other RhoGEF family is characterized by two conserved domains called DOCK homology region (DHR) 1 and 2 (Rossman et al. 2005).

Surprisingly no $\mathrm{DH}$ or $\mathrm{PH}$ domains have yet been reported in plants. Moreover, Arabidopsis contain a single gene encoding a Dock-like protein called SPIKE1 (SPK1) (Qiu et al. 2002; Basu et al. 2008). This protein, homologous to the human DOCK family RhoGEFs (Côte and Vuori, 2007), was shown to control actin-dependent morphogenesis through the heteromeric WAVE and ARP2/3 complexes (Basu et al. 2008; Qiu et al. 2002).

However, recently, a specific family of plant proteins, carrying the PRONE- (plantspecific ROP nucleotide exchanger) domain exhibiting GEF-activity, has been revealed through yeast two-hybrid screens using dominant negative ROP mutants as bait (Berken et al. 2005; Gu et al. 2006).

ROPGEFs are abundant proteins in plants and are differentially expressed in diverse tissues (Berken et al. 2005; Gu et al. 2006; Kaothien et al. 2005). Five out of the 14 PRONE- 
containing Arabidopsis GEFs are specifically expressed or enriched in pollens (Zhang and McCormick 2007). These proteins have a specific conserved C-terminus implicated in the regulation of their activity (Zhang and McCormick 2007). Among the other ROPGEF genes there are two which are root specific while the others are expressed in vegetative organs as determined by RT-PCR analysis (Qiu et al. 2002).

In Arabidopsis, over-expression of ROPGEF1 in pollen tubes leads to the depolarization of pollen tube growth which is similar to the constitutively active ROP1 mutant phenotype (Gu et al. 2006). Expressions of a dominant negative (DN) ROP1 mutant suppress the ROPGEF1 overexpression phenotype, supporting a role for ROPGEF1 as an upstream activator of ROP1.

In animal cells, a number of RhoGEFs are phosphorylated by RTKs (receptor tyrosine kinases), leading to activation or inactivation of downstream Rho GTPases (Noren and Pasquale 2004; Van Aelst and Cline 2004). Receptor tyrosine kinases are a large and diverse family of cell surface receptors that play important roles in fundamental cell processes (Schlessinger 2000). In tomato, a ROPGEF termed KPP (kinase partner protein) was isolated in a yeast two-hybrid screen using a pollen-specific tomato receptor-like serine/threonine kinase (pollen receptor-like kinase, LePRK) (Kaothien et al. 2005). KPP interact with the cytoplasmic domain of LePRK1 and 2 and was shown to be phosphorylated in vivo which suggest that ROPGEFs may be substrates of receptor-like kinases (RLKs).

\subsection{ROP GTPase effectors and their involvement in various signaling pathways}

Exchange of the hydrolyzed GDP for GTP results in a conformational change of GTPases, unmasking structural domains by which they can bind to their target proteins, the effectors.

Many effectors of yeast and animal R-type GTPases are well known (Cotteret and Chernoff 2002). Rho and CDC42/RAC GTPases bind different effectors and are involved in specific but interlinked signaling cascades in animal cells (Bustelo et al. 2007). Due to the early split of viridiplantae from the animal-fungal-amoebozoa lineage, Rho-family genes in plants have developed novel features and new modes of regulation as well as recruited specific effectors (Brembu et al. 2006, Berken and Wittinghofer 2008). In plants, a single group of Rho-type GTPases, the ROP proteins, has to fulfill all of Rho functions, and it can even be hypothesized that certain roles of the missing mitogen-activated RAS proteins are also played by ROPs in plants (Yang and Fu 2007). 
Table 2: ROP effectors and their experimentally indicated interactions with ROPs (only Arabidopsis genes are given, even if the interaction of homologous proteins was detected in other species) (Feher et al. 2008)

\begin{tabular}{|c|c|c|c|c|c|}
\hline Annotation & Name & AGI & Affy ID & Interaction & Reference \\
\hline \multirow{11}{*}{$\begin{array}{l}\text { ROP-interactive CRIB motif- } \\
\text { containing protein }\end{array}$} & RIC1 & At2g33460 & 255837_at & At ROP1, 2, 4 & $\begin{array}{l}\text { Wu et al. } 2001, \\
\text { Fu et al. } 2005\end{array}$ \\
\hline & RIC2 & At1g27380 & 264495_at & & \\
\hline & RIC3 & At1g04450 & & At ROP1 & $\begin{array}{l}\text { Wu et al. } 2001, \\
\text { Gu et al. } 2005\end{array}$ \\
\hline & RIC4 & At5g16490 & 250120_at & At ROP1, 2, 4 & $\begin{array}{l}\text { Wu et al. } 2001, \\
\text { Gu et al. } 2005, \\
\text { Fu et al. } 2005\end{array}$ \\
\hline & RIC5 & At3g23380 & & At ROP1 & Wu et al. 2001 \\
\hline & RIC6 & At2g20430 & 257397_at & & \\
\hline & RIC7 & At4g28560 & 253769_at & & \\
\hline & RIC8 & At1g03982 & & & \\
\hline & RIC9 & At1g61795 & & & \\
\hline & RIC10 & At4g04900 & 255307_at & & \\
\hline & RIC11 & At4g21745 & & At ROP11 & \\
\hline KLUNKER-like & PIROGI & At5g18410 & 250041_at & AtROP2 & Basu et al. 2004 \\
\hline Cinnamoyl-CoA reductase & CCR1 & At1g15950 & 261792_at & OsRAC1 & Kawasaki et al. 2006 \\
\hline UDP-glucosyl transferase & UGT1 & At1g05560 & 263184_at & AtROP1 & Hong et al. 2001 \\
\hline \multirow{10}{*}{ Respiratory burst oxidase } & ATRBOHA & At5g07390 & 250629_at & OsRac2, 5,7 & Wong et al. 2007 \\
\hline & ATRBOHB & At1g09090 & 264647_at & OsRac1, 2, 5, 7 & Wong et al. 2007 \\
\hline & ATRBOHC & At5g51060 & 248486_at & OsRac2, 6, 7 & Wong et al. 2007 \\
\hline & ATRBOHD & At5g47910 & 248719_at & OsRac2, 3, 6, 7 & Wong et al. 2007 \\
\hline & ATRBOHE & At1g19230 & 256011_at & n.t. & \\
\hline & ATRBOHF & At1g64060 & 262344_at & n.t. & \\
\hline & ATRBOHG & At4g25090 & 254092_at & n.t. & \\
\hline & ATRBOHH & At5g60010 & 247647_at & n.t. & \\
\hline & ATRBOHI & At4g11230 & 254912_at & n.t. & \\
\hline & ATRBOHJ & At3g45810 & 252544_at & n.t. & \\
\hline \multirow{5}{*}{$\begin{array}{l}\text { Wiskott-Aldrich syndrome } \\
\text { protein-family }\end{array}$} & WAVE1/SCAR1 & At2g34150 & 256721_at & AtROP5, 8, 11 & Uhrig et al. 2007 \\
\hline & WAVE2/SCAR4 & At1g29170 & & AtROP2, 5, 7, 8, 11 & Uhrig et al. 2007 \\
\hline & WAVE3/SCAR4 & At5g01730 & 251105_at & AtROP7, 8, 11 & Uhrig et al. 2007 \\
\hline & WAVE4/SCAR2 & At2g38440 & 265275_at & AtROP5, 8, 11 & Uhrig et al. 2007 \\
\hline & WAVE5/SCAR-like & At4g18600 & 254621_at & AtROP8 & Uhrig et al. 2007 \\
\hline \multirow{7}{*}{$\begin{array}{l}\text { Recptor-like cytoplasmic } \\
\text { protein kinase }\end{array}$} & AtRLCK VI_A1 & At5g57670 & $\begin{array}{l}247872 \_ \text {at } \\
\text { 247920_at }\end{array}$ & & \\
\hline & AtRLCK VI_A2 & At2g18890 & 266946_at & & \\
\hline & AtRLCK VI_A3 & At5g65530 & 247170_at & & \\
\hline & AtRLCK VI_A4 & At5g10520 & 250443_at & AtROP4 & Molendijk et al. 2008 \\
\hline & AtRLCK VI_A5 & At5g35960 & 249676_at & & \\
\hline & AtRLCK VI_A6 & At3g05140 & 259350_at & AtROP4 & Molendijk et al. 2008 \\
\hline & AtRLCK VI_A7 & At5g18910 & 249950_at & & \\
\hline $\begin{array}{l}\text { Novel cystein-rich protein } \\
\text { kinase }\end{array}$ & NRCK & At2g28250 & 265545_at & AtROP4, 11 & Molendijk et al. 2008 \\
\hline Tropomyosin-related protein & ICR1 & At1g17140 & 262538_at & AtROP6, 10 & Lavy et al. 2007 \\
\hline Required for Mla12 resistance & RAR1 & AT5G51700 & 248379_at & OsRac1 & Thao et al. 2007 \\
\hline Heat shock protein 90 & Hsp90 & At5g52640 & 248332_at & OsRac1 & Thao et al. 2007 \\
\hline
\end{tabular}


Therefore, although that some of the basic functions of Rho GTPases are common in all eukaryotes (e.g. the regulation of cell polarity, morphogenesis and reactive oxygen species (ROS) production), the precise molecular mechanisms are not well conserved. Moreover, ROPs are also involved in plant-specific processes, such as cell wall synthesis. That is why the list of ROP effectors is strikingly different from that of the ophistokonta (metazoa and fungi) Rho GTPases (Cotteret and Chernoff 2002).

In Table 2, the presently known ROP effectors are summarized. Their roles in different biological processes are discussed below in details.

\subsubsection{ROPs as regulators of the actin cytoskeleton, vesicular trafficking and polarity establishment}

Cell polarity, broadly defined as asymmetry within a cell, is a fundamental feature of cell function that is tied to developmental and environmental regulation. Polarity formation is initiated by a polarizing signal, which regulates polar distribution of signaling molecules and leads to polarity establishment and maintenance through the cytoskeleton and vesicular trafficking. In plants, cell polarity is fundamental to development, growth, and morphogenesis in every stage of the life cycle. Zygote polarization is required for the first asymmetric division to the embryo axis. Similarly, polarized cell division is essential for cell differentiation, organ initiation, and morphogenesis. Polarized cell expansion generates cells with specific shapes that are associated with specialized functions, e.g., polarized and guided pollen tube growth for sperm delivery. Finally, polar localization of auxin-carrier proteins allows directional auxin flow and auxin-gradient formation, processes that are essential for organ and tissue formation and growth.

Numerous studies showed that plant cells use conserved mechanisms, to coordinate cytoskeleton dynamics/reorganization and vesicular trafficking required for polarity establishment and maintenance.

ROPs are apparently important for polarity establishment during embryogenesis because up-regulating their activity induces seedling phenotypes suggestive of defective embryonic development, such as the lack of shoot or root axis or the development of fused cotyledons (Tao et al. 2002).

It is well known that ROPs play an important role in polarity determination in tip-growing pollen tubes and root hairs (Yang 2002; Gu et al. 2004; Kost et al. 1999; Li et al. 1999, Lin and Yang 1997). Studies using the pollen-specific AtROP1 and its homologues showed that ROP is 
localized and selectively activated at the apical region of the tube plasma membrane and its activity controls tip growth in pollen tubes (Gu et al. 2003; Hwang et al. 2005). Blocking AtROP1 signaling by expressing dominant-negative ROP1 or by microinjection anti-ROP1 antibody inhibited pollen tube tip growth, while expressing constitutively active (CA) form induced isotropic growth (Gu et al. 2003; Hwang et al. 2005).

Recently, it has been shown that pollen tube growth requires a balance of lateral propagation and global inhibition of ROP activity (Hwang et al. 2009). By manipulating the ROP activity level, it was shown that the plasma membrane distribution of ROP activity as an apical cap determines the tip growth region and that efficient tip growth requires an optimum level of the apical ROP1 activity. Excessive ROP activation induced the enlargement of the tip growth region, causing growth depolarization and reduced tube elongation. The data showed that the apical ROP1 cap is generated by lateral propagation of a localized ROP activity. Moreover, the lateral propagation of apical ROP1 activity is limited by the RhoGDI- and RhoGAP-mediated global inhibition. It is proposed that the balance between the lateral propagation and the global inhibition maintains an optimal apical ROP1 cap and generates the apical ROP1 activity oscillation required for efficient pollen tube elongation (Hwang et al. 2009).

ROPs affect the cytosolic $\mathrm{Ca}^{2+}$ concentration, an important regulator for the actin cytoskeleton, and disrupt the tip-focused $\mathrm{Ca}^{2+}$ gradient known to be crucial for the polar cell growth process (Yang 2002; Gu et al. 2004; Hepler et al. 2001). It was shown that an Arabidopsis GTPase (AtROP5 or At-RAC2) associates with phosphatidylinositol monophosphate kinase (PtdInsP-K), co-localizes with its product, phosphatidylinositol (4,5)-bisphosphate $\left(\operatorname{PtdIns}(4,5) \mathrm{P}_{2}\right)$, at the tobacco pollen tip membrane and is involved in the regulation of calcium fluxes and actin dynamics. Thereby, data provide a link between the GTPase and intracellular $\mathrm{Ca}^{2+}$ regulatory mechanisms (Kost et al. 1999).

Moreover, ROPs appear to influence the phosphorylation state of proteins of the actin depolymerizing factor (ADF/cofilin family). ADF/cofilin homologues, which are well characterized in plants, stimulate actin depolymerization and recycling of monomers, and generate extendable filament ends by severing of preexisting filaments as well. Their activity is partly controlled by inhibitory phosphorylation that has been documented to be ROP-regulated in pollen tubes (Chen et al. 2003). 
There is evidence suggesting that in plant systems calcium dependent protein kinases (CDPK) are involved in ADF phosphorylation (Allwood et al. 2002).CDPK represent a very large family of Ser/Thr protein kinases in plants. Early studies in maize showed that plant extract enriched in CDPK was able to phosphorylate ADF indicating that maize ADF is controlled indirectly by $\mathrm{Ca}^{2+}$ (Smertenko et al. 1998; Allwood et al. 2002).

In addition, ROPs affect the actin cytoskeleton by interacting with the SCAR/WAVE complex which activates the actin-nucleating protein complex ARP2/3 (Smith and Oppenheimer 2005; Deeks and Hussey 2005). The transgenic plants overexpressing dominant negative (DN) AtROP2 show mild leaf epidermal pavement cell shape defects and subtle changes in the actin cytoskeleton relative to wild-type cells (Yang 2002; Gu et al. 2006; Fu et al. 2005). Similar defects in pavement cells and distorted trichome phenotype are induced by mutations in the SCAR/WAVE ARP2/3 pathways (Szymanski 2005).

In addition to the ARP2/3 complex, plants also contain homologues of diaphanous-related formins (DRFs), actin nucleators, that operate as Rho effectors in animals and yeast to induce actin polymerization upon binding of Rho to its GTPase binding domain (Deeks et al. 2002). Formins are a diverse group of proteins defined by the presence of two regions of sequence homology termed as formin-homology $(\mathrm{FH})$ regions. Arabidopsis has at least 23 proteins with $\mathrm{FH}$ domains, some of them with an unusual structure indicating that they are inserted into a membrane, which could be related to a plant-specific function of these proteins (Cvrckova 2000; Deeks et al. 2002). Overexpressing of the Arabidopsis formin AFH1 stimulated actin cable formation in pollen tubes and resulted in depolarized phenotypes similar to those induced by overexpressing WT or CA forms of ROP (Cheung and Wu 2004). This might indicate that ROPs and formins function in the same pathway but there are no data about a direct link between formins and ROPs.

Plants have a family of small CRIB-containing proteins called RICs (Rho-interacting CRIB-motif containing proteins). The CRIB motif is required for their specific interaction with GTP-bound ROP1 (Wu et al. 2001). RIC proteins have been implicated in actin polymerization, microtubule bundling, $\mathrm{Ca} 2+$ fluxes during polar growth, cell expansion and morphogenesis $(\mathrm{Gu}$ et al. 2005; Hwang et al. 2005; Fu et al. 2001). RICs are represented by eleven members in Arabidopsis and are highly divergent in sequence outside of the CRIB region. Over-expression of 
various RIC proteins in pollen tubes causes distinct phenotypes indicating specific functions for the individual RICs (Wu et al. 2001).

Intracellular localization and co-expression of ROP and RIC proteins in plant cells indicate that RICs are downstream targets of ROP GTPases. It has been shown that an Arabidopsis ROP GTPase (AtROP1) activates RIC3 in pollen tubes and induces a high $\mathrm{Ca}^{2+}$ concentration condition at the tip region that leads to actin disassembly (Gu et al. 2005). AtROP1 also activate RIC4 that promotes F-actin assembly ( $\mathrm{Gu}$ et al. 2005). ROP regulates two counteractive actin-regulating pathways that allow the control of actin dynamics at the elongating pollen tube tip (see Figure 2) RIC4 shows an oscillatory pattern of tip membrane localization (implying oscillatory activation of AtROP1) that correlates with the periodicity of pollen tube growth (Gu et al. 2005; Hwang et al. 2005). This oscillatory activation of AtROP1 is in phase with RIC4-promoted F-actin assembly, ahead of growth and the RIC3-regulated accumulation of $\mathrm{Ca}^{2+}$ in the tip, that provide a temporal relationship between these cellular activities and growth ( $\mathrm{Gu}$ et al. 2005). Moreover, recently it has been demonstrated that RIC4-dependent actin assembly is required for polar vesicle accumulation, whereas RIC3-mediated actin disassembly is required for exocytosis. Thus ROP1-dependent F-actin dynamics controls tip growth through spatiotemporal coordination of vesicle targeting and exocytosis (Lee et al. 2008).

That RICs are indeed downstream targets of ROP GTPases could also be demonstrated during the establishment of epidermal cell shape in Arabidopsis by the concerted action of AtROP2/4, AtRIC1 and 4 (Fu et al. 2005). AtROP2/4 GTPase, when activates RIC4 promote actin microfilament formation in the region of growing lobes, whereas through the activation of RIC1 they promote microtubule bundling at neck regions. These cytoskeletal changes act as signals to direct the direction of cell wall growth (Fu et al. 2005).

Another scaffold protein serving as ROP effector ICR1 (Interactor of Constitutively active ROP) act in the regulation of the jigsaw puzzle-like pavement cell shape. Knocking down ICR1 caused the jigsaw puzzle pavement cells to become cubical or elongated in the adaxial epidermis of leaves (Yang and Fu 2007). This effector has recently also been implicated in the establishment of the polarity in auxin transport (Hazak and Yalovsky 2010; and see further).

Rho GTPase is also known to regulate the exocytosis and endocytosis processes. A role for exocytosis/endocytosis in cell polarity formation has been established in various eukaryotic systems (Brennwald and Rossi 2007; France et al. 2006; Mehta et al. 2005; Zajac et al. 2005). 
Polarized exocytosis allows for tip growth in root hair and pollen tube by supplying membrane and wall materials. A recently discovered interacting partner of the active AtROP1 GTPase, ICR1, serve as an adaptor protein linking ROP GTPase signaling to the exocyst complex involved in exocytosis-related vesicle trafficking and membrane fusions (Lavy et al. 2007). ICR1 functions as a scaffold that interacts with diverse but specific group of proteins including SEC3 subunit of the exocyst vesicle tethering complex. ICR1-SEC3 complex can interact with ROPs in vivo and are thereby recruited to the plasma membrane (Bloch et al. 2008).

The ectopic expression of a constitutive active Arabidopsis RAC, AtRAC10, disrupts actin cytoskeleton organization and membrane cycling (Bloch et al. 2005). The activated AtRAC10 induced deformation of root hairs and leaf epidermal cells. Moreover, endocytosis and membrane recycling were inhibited in activated AtRAC10 transgenic plants.

The functional role of ROPs in polarity determination relies on their combined actions on membrane trafficking and cytoskeleton organization within the cells.

\subsubsection{ROPs as regulators of auxin signaling through ubiquitin/26S proteasome-mediated proteolysis}

It is well known that auxin is critical for multiple cellular processes in plants, like cell division, growth, and differentiation, and its action underlies developmental processes ranging from embryogenesis to reproduction. The auxin signaling relies on ubiquitin ligase SCFTIR1mediated $26 \mathrm{~S}$ proteasome-dependent proteolysis of a large family of short-lived transcription regulators, the Aux/IAA (auxin/indole acetic acid-inducible) proteins, which leads to derepression of auxin-responsive genes (Rogg and Bartel 2001; Hagen and Guilfoyle 2002; Hellmann and Estelle 2002).

ROP GTPases were shown to be activated by auxin and in turn to stimulate auxin responsive gene expression (Tao et al. 2002). Moreover, it was reported that a ROP GTPase (NtRac1) act as a regulator for ubiquitin/26S proteasome-mediated proteolysis (Tao et al. 2005). Increasing ROP signaling activity promotes Aux/IAA degradation, whereas the downregulation of that activity results in the reduction of auxin-accelerated Aux/IAA proteolysis. ROP GTPases mediate the recruitment of nucleoplasmic Aux/IAAs into proteolytically active nuclear protein bodies, into which components of the SCFTIR1, COP9 signalosome, and 26S proteasome are also recruited (Tao et al. 2005). These data provide strong evidence that ROP GTPases are 
integral components of the auxin-signaling pathway of Aux/IAA degradation and auxinresponsive gene expression.

In a recent publication, the involvement of the ROP GTPase associated scaffold protein "Interactor of Constitutive active ROP 1" (ICR1) was shown in the recruitment of the auxin efflux carrier PIN proteins to polar domains in the plasma membrane (Hazak et al. 2010). Directionality of auxin transport depends on polar subcellular distribution of PINFORMED (PIN) family of efflux transporters (Galweiter et al. 1998; Wisniewska et al. 2006). PIN polarity is a result of constitutive endocytic recycling. icrl mutant plants and embryos have severe developmental aberrations that are caused by abnormal auxin distribution. ICR1 functions at or close to the plasma membrane where it is required for exocytosis. ICR1 transcription is quickly induced by auxin but is post-transcriptionally repressed at the site of stable auxin maximum in embryos and roots. These results imply that ICR1 is part of an auxin-regulated feedback loop, integrating auxin mediated gene expression with ROP-modulated cell polarity (Hazak and Yalovsky 2010).

\subsubsection{ROPs role in ABA signaling}

ROP GTPases are known to be important negative regulators of abscisic acid (ABA) signaling (Lemichez et al. 2001; Zheng et al. 2002). An Arabidopsis ROP GTPase (AtROP6) is inactivated by abscisic acid and plays an important role in stomatal closure (Lemichez et al. 2001). Two ROPs, AtROP6 and AtROP10, can suppress ABA-induced responses, like actin reorganization in guard cells, stomatal closure, seed germination, root elongation and gene expression. Moreover, overexpression of DN forms of these ROPs overcomes the ABAinsensitivity in abil-1 mutants, supporting the idea that these small GTPases act as negative regulators of the ABI1-1-mediated ABA signaling pathway (Lemichez et al. 2001; Zheng et al. 2002). AtROP10 is a negative regulator of ABA responses and regulates ABA induction of a subset of genes associated with stress responses (Xin et al. 2005).

\subsubsection{ROPs as regulators of NADPH oxidase and pathogene defence}

Plants can generate extracellular superoxide via plasma membrane (PM)-localized NADPH oxidases (NOXs), which are homologous to the catalytic subunit (gp91phox) of mammalian phagocyte NOX. Plant NADPH oxidase genes, termed rboh (for respiratory burst oxidase homolog), have been isolated from many plants species, including rice (Oryza sativa), 
Arabidopsis thaliana, tobacco (Nicotiana benthamiana), and potato (Solanum tuberosum) (Groom et al. 1996; Keller et al. 1998; Torres et al. 2002; Yoshioka et al. 2001; Yoshioka et al. 2003; Sagi and Fluhr 2006).

In Arabidopsis thaliana 10 NOX genes were identified (Foreman et al. 2003). They catalyse the extracellular formation of the superoxide anion $\left(\mathrm{O}_{2}{ }^{-}\right)$from molecular oxygen using NADPH as an electron donor (Sagi and Fluhr 2001). Extracellular superoxide readily gives rise to other reactive oxygen species (ROS) including hydrogen peroxide, by dismutation, and the hydroxyl radical via the Fenton reaction (Halliwell and Gutteridge 1989). Hydrogen peroxide readily crosses the plant plasma membrane through water channels (Henzler and Steudle 2000) and can subsequently act as an intracellular (Kovtun et al. 2000) and intercellular (Allan and Fluhr 1997) signaling molecule. Reactive oxygen species (ROS) produced by NADPH oxidase have been shown to play many important roles in signaling and development in plants, such as plant defense response, cell death, abiotic stress, stomatal closure, and root hair development (Baxter-Burrell et al. 2002; Torres et al. 2002; Foreman et al. 2003; Kwak et al. 2003; Yoshioka et al. 2003; Jones et al. 2007).

Although there are evidences for the involvement of RBOHs in ROS production in many aspects of signaling and development in plants (Apel and Hirt 2004, Gapper and Dolan 2006), the regulation of plant RBOHs is still unclear (Apel and Hirt 2004; Torres and Dangl 2005). Strong evidences suggest, however, that ROPs regulate plant NOX activity.

The NADPH oxidase activity responsible for the apical accumulation of reactive oxygen species (ROS) was shown to be important in root hairs (Foreman et al. 2003) and in pollen tubes elongation (Potocky et al. 2007). ROS are considered to be essential for the establishment of a tip-focused $\mathrm{Ca}^{2+}$ gradient required for root hair growth, through the stimulation of plasma membrane $\mathrm{Ca}^{2+}$ channels (Foreman et al. 2003). In addition to presumably regulating actin organization and membrane trafficking, elevated $\mathrm{Ca}^{2+}$ levels at the root hair apex were recently demonstrated to stimulate NADPH oxidase activity. This creates a positive feedback loop that is likely to contribute to the maintenance of the polarity of root hair growth (Takeda et al. 2008). Root epidermal cells of Arabidopsis scnl mutants missing a RhoGDI isoform are unable to polarize ROP activity and consequently fail to form normal root hairs. ROS production is also enhanced and depolarized in these cells, suggesting that NADPH oxidase activity required for root hair formation is under the control of ROP GTPases (Carol et al. 2005). 
In addition, a constitutively active form of cotton small GTPase that is highly expressed during cotton fiber development has been shown to induce ROS production in cultured Arabidopsis and soybean (Glycine max) cells (Potikha et al. 1999). This suggests that this GTPase activates a NOX protein during the rapid elongation of cotton fibers.

GTPases also spatially control non growth-related processes associated with differentiation. During the development of $Z$. elegans xylem tracheary elements, NOX-derived ROS are required for formation of the highly lignified banded thickenings in the cell wall (RosBarcelo et al. 2002). The ROS are produced in adjacent xylem parenchyma cells and not in the differentiating tracheary elements themselves (Nakanomyo et al. 2002; Ros-Barcelo 2005).

Furthermore, loss of function of the GAP ROPGAP4 reduces tolerance too hypoxia stress in Arabidopsis as a result of super-activation of ROP signaling (Baxter-Burrell et al. 2002). Analysis of this mutant has revealed a feedback mechanism whereby sufficient ROS are produced to induce hypoxia response so that protection against anoxia is ensured but excessive ROS accumulation that would lead to oxidative stress is prevented; this has lead to the suggestion that ROPs act as a molecular rheostat sensing oxygen deprivation (Baxter-Burrell et al.2002).

ROP GTPases have been reported to directly bind to and activate NADPH oxidase subunits (Wong et al. 2007). A direct interaction of the rice (Oryza sativa L.) ROP GTPase OsRAC1 and the conserved N-terminal region of OsRBOHA-D proteins, the rice homologues of the gp91phox catalytic subunit of the NADPH-oxidase complex, has been established (Wong et al. 2007).

ROP regulated NOX activity in plants play important role in pathogene defence. OsRAC1 stimulates cinnamoyl-CoA reductase, an enzyme required for the biosynthesis of lignin, which is important to block pathogene infection (Kawasaki et al. 2006). Cinnamoyl-CoA reductase appears to interact directly with OsRAC1 because this small GTPase activates the lignin biosynthesis enzyme in vitro and these two proteins interact in yeast two hybrid assays. OsRAC1 also suppresses the expression of methalothinein that scavanges ROS (reactive oxygen species) further enhancing the ROS-signaling towards the defense response (Wong et al. 2004).

OsRAC1 was also shown to be a positive regulator of resistance to fungal and bacterial pathogens in rice (Ono et al.2001). OsRAC1 forms a complex with MAPK6, RAR1, SGT1, Hsp90 and RACK1, thereby transmitting signals to downstream components of innate immunity 
(Lieberherr et al. 2005, Thao et al. 2007, Nakashima et al. 2008, Fujiwara et al. 2009). Dominantnegative OsRAC1 impairs $N$ gene-mediated resistance in tobacco (Moeder et al. 2005).

Maize ROPs induce ROS production when expressed in mammalian cells (Hassanain et al. 2000). In addition, ROP small GTPases are up-regulated in pathogen-inoculated chickpea leaves and elicitor treated cell cultures (Ichinose et al. 1999). Transformation with constitutively active cotton RAC13 also increases ROS production in soybean and Arabidopsis cell cultures (Potikha et al. 1999). Moreover, human RAC promotes an oxidative burst in soybean cell cultures (Park et al. 2000). Silencing of ROP in tobacco by heterologous expression of Medicago Ms-Racl antisense cDNA causes defects in defense responses (Schiene et al. 2000). Arabidopsis ROP2 promotes ROS production and is involved in phosphatidic acid-induced cell death (Park et al. 2004).

In barley, HvRACB is a negative regulator of disease resistance to a powdery mildew pathogene (Schultheiss et al. 2002, Schultheiss et al. 2003). The closest ortholog of HvRACB in rice, OsRACB, also known as OsRAC6 (Miki et al. 2005), was proposed as a negative regulator in disease resistance (Jung et al. 2006). Similarly, tobacco NtRAC5 is considered as a negative regulator of NADPH oxidase involved in ROS production in tobacco (Morel et al. 2004). There are seven RAC/ROP members in rice (Miki et al. 2005), among which only OsRAC1 (Ono et al. 2001) and OsRACB (Jung et al. 2006) had been previously associated with defense.

\subsubsection{ROPs effectors in cell wall synthesis}

An Arabidopsis ROP GTPase has been shown to bind a UDP-glucose transferase (UGT1) and via this interaction may indirectly regulate callose synthase activity and the formation of the new cell wall during cytokinesis. UGT1 transfers UDP-glucose to the catalytic subunit of callose synthase (Hong et al. 2001). Another plant-specific ROP effector, cinnamoyl-CoA reductase (CCR), has also a role in cell wall formation, namely, it catalyses the NADPH-dependent synthesis of lignin monomers (Thao et al. 2007)

\subsubsection{ROPs and kinases}

In spite of the high level of structural similarity of plant ROP GTPases to mammalian $\mathrm{CDC} 42 / \mathrm{RAC}$ proteins, there are considerable differences as their possible effectors are concerned (Cotteret and Chernoff 2002, and see above). One of the most characteristic differences is that in plants no protein kinases carrying the CRIB-motif and directly activated by ROP GTPases could 
be identified. In animal cells, mitogenic signals are mediated by RAS that activate the RAFMEKK-MAPK protein kinase cascade, while CDC42 and RAC activate another specific class of protein kinases, the members of the p21-activated kinase (PAK) family, containing the CDC42/RAC interactive binding (CRIB) motif (Zhao and Manser 2005; Bokoch 2003). While the RAS-RAF-MEKKMAPK pathway may be specific to metazoa, PAK kinases regulated by CDC42 are present also in yeast; with MAP kinase cascades among their effectors (Dan et al. 2001).

The lack of RAS from plants as well as the significance of animal and yeast CDC42/RAC proteins in protein kinase signaling strongly support the hypothesis that ROPs are also linked to protein kinase cascades in plants.

ROPs have also been implicated in the regulation of gene expression and certain pathways may involve MAPK cascades. In rice, OsRAC1 was shown to be required for OsMAPK6 protein accumulation and activity in response to elicitor treatment, indicating the role of OsRAC1, and potentially other ROPs, in the activation of MAP-kinase cascades (Lieberherr et al. 2005). In the same study, it was also proved that the constitutively active OsRAC1 protein occurs in the same protein complex together with Os-MAPK6. However, no direct interaction is evident.

Recently, our group as well as a group in Germany have reported the direct interaction of plant ROP GTPases with receptor-like cytoplasmic kinases (RLCK class VI) from Arabidopsis (Molendijk et al. 2008) and alfalfa (Dorjgotov et al. 2009).

This kinase family has 14 members belonging to two groups in Arabidospsis (Jurca et al. 2008). The Arabidopsis RLCK-VI genes are rather ubiquitously expressed in plant development, but some members are strongly expressed in the pollen (Molendijk et al. 2008; Jurca et al. 2008). In addition, the expression of one of the ROP-interacting RLCK VI kinases was shown to be predominantly associated with vasculature and upregulated in leaves exposed to pathogens (Molendijk et al. 2008).

A further kinase designated as cysteine-rich receptor kinase (NCRK) belonging to a distinct kinase family has also been shown to interact with ROPs (Molendijk et al. 2008). None of these plant specific ROP-interacting kinases has any characteristic domain or motif that could be correlated with their ability to bind ROP GTPases. 
AtROP5 (AtRAC2) was found to physically associate with a phosphatidylinositol monophosphate kinase (PtdIns PK) activity (Kost et al. 1999). This observation indicates that lipid kinases can also be potential ROP effectors in plants similarly as in animal cells (Oude et al. 2004), although the direct interaction of this type of kinases with ROPs needs to be experimentally verified.

\subsection{Plant Receptor-like Kinases}

Plants have to respond and adapt to a variety of continuously changing environmental factors in order to establish an appropriate developmental strategy to ensure survival. There are ample data showing that protein phosphorylation/dephosphorylation plays a central role in cellular signal transduction in all organisms (Herrmann et al. 2006; Stone and Walker 1995).

Interestingly, plants have a similar system as mammals to detect and transfer signals across the cell wall into the nucleus where adaptations could be initiated. For the detection and transfer of an external signal, mammalian systems have receptor protein kinases. The proteins contain a single hydrophobic transmembrane domain, an extracellular domain and protein kinase domain. The majority of animal receptor kinases are phosphorylated on tyrosine residues within the kinase domain (receptor tyrosin kinases or RTKs; Ullrich and Schlessinger 1990), but a few were discovered which are phosphorylated on serine and threonine residues (Lin et al. 1992).

In plants, two different types of transmembrane receptor kinases are known, including receptor-like serine/threonine (Ser/Thr) kinases (receptor-like kinases RLKs; Shiu and Bleecker 2001, 2003; Shiu et al. 2004; Walker 1994), structurally similar to mammalian RTKs, and receptor histidine (His) kinases (Grefen and Harter 2004; Mizuno 2005; Urao et al. 2000).

Since the first RLK-encoding gene family was found in Zea mays (Walker and Zhang 1990), thousands of RLK genes have been identified from many different plant species. The Arabidopsis genome contains more than 600 members, representing nearly $2.5 \%$ of the annotated protein-coding genes; and more than 1000 members were annotated in the rice genome (Shiu et al. 2004).

\subsubsection{Classification of plant receptor-like kinases (RLKs)}

On the basis of the presence/absence and identity of the extracellular domains and the phylogeny of kinase domains, the RLK family is subdivided into 46 different subfamilies (Shiu and Bleecker 2003). Among the RLKs, the majority (75\% in Arabidopsis) has a signal percepting 
domain (amino-terminal extracellular domain), a transmembrane domain, and a carboxylterminal kinase domain; the rest are defined as receptor-like cytoplasmic kinase because they have a carboxyl-terminal kinase domain but no apparent signal perception or transmembrane domains (Shiu and Bleecker 2001).

Based on sequence and domain organization among the extracellular domains, the RLKs have been classified into 15 types: CRINKLY4-like, Ctype lectin-like, CrRLK1-like, DUF26-like, extensin-like, legume (L)-lectin-like, LRK10-like, LRR-like, LysM-like, PERK-like, RKF3-like, S-domain-like, thaumatin-like, URK1-like, and WAK-like (Haffani et al. 2004). LRR-RLK represents the largest group of RLK in Arabidopsis and it is divided into 13 subfamilies (LRR I to XIII) with 216 members.

The rest of RLK family (25\%) are Receptor-like cytoplasmic kinases (RLCKs). The RLCKs are represented by 12 families (RLCK I to XII) with 193 protein coding genes.

\subsubsection{Functions of receptor-like cytoplasmic kinase (RLCK) proteins}

The extracellular domain allows RLKs to respond to multiple external signals. Anyway, the majority of these proteins have not been assigned a biological function as receptors so far, and the attribute "receptor-like" indicates the tentative character of their functional classification.

As suggested by the diversity and size of the RLK/Pelle family, its members are involved in diverse processes in plants. It has been shown that they play role in the regulation of meristem proliferation (Clark et al. 1997; DeYoung et al. 2006), organ specification (Torii et al. 1996; Muto et al. 2004; Shpak et al. 2004; Chevalier et al., 2005; Kwak et al. 2005; Eyuboglu et al. 2007), reproduction (Albrecht et al. 2005; Colcombet et al. 2005; Escobar-Restrepo et al. 2007), and hormone signal transduction (Li and Chory 1997; Li et al. 2002; Nam and Li 2002; Zhou et al. 2004). Other RLK/Pelle members function in signaling networks involving environmental stimuli, both abiotic (Sivaguru et al. 2003; Hou et al. 2005) and biotic (Song et al. 1995; GomezGomez and Boller 2000; Endre et al. 2002; Stracke et al. 2002; Chen et al. 2003; Fontes et al. 2004; Sun et al. 2004; Diener and Ausubel 2005; Florentino et al. 2006; Zipfel et al. 2006; Acharya et al. 2007; Miya et al. 2007). In some cases, RLK/Pelle genes exhibit multifunctionality, playing roles in both development and defense (Bent and Mackey 2007).

However, in plants, only a small number of ligand/receptor pairs have been well characterized. In Arabidopsis, the well characterized pair CLV3/CLV1 (Clark et al.1997; Ogawa et al. 2008) was shown to be important for meristem regulation, BRI1 (Li and Chory1997) is 
involved in growth promotion; IDA/ HAESA (Butenko et al. 2009) in abscission and Flagellin/FLS2 (Chinchilla et al. 2006; Gomez-Gomez and Boller, 2000), EF-Tu/ EFR (Kunze et al. 2004; Zipfel et al. 2006) and AtPep1/PEPR1 (Yamaguchi et al. 2006) in the immune response.

Concerning the potential role of RLCKs in signal transduction pathways, only limited information is available. In tomato, Pto (RLK) confers resistance to the pathogen Pseudomonas syringae (Tang et al. 1996) and it is mediated by the RLCK, Pto-interacting1 (Pti1, RLCK VIII) (Zhou et al. 1995). More interestingly, Ptil-like proteins have recently been shown to play a role in phospholipid and oxidative stress signaling downstream of PDK and OXI1 kinases (Anthony et al. 2006, Rentel et al. 2004). Ptil-like kinases have also been identified in maize and one of them proved to be specifically expressed in the pollen (Herrmann et al. 2006).

In Arabidopsis, the RLCK kinase PBS1 (class VII), and the RLK kinase RPS5, are both required to recognize the Pseudomonas syringae strains that express the avirulence gene avrPphB (Swiderski and Innes 2001).

Self-incompatibility is mediated by the SRK kinase (RLK) the function of which is linked to the presence of MLPK (RLCK VII) (Murase et al. 2004).

The overexpression of the Constitutive Differential Growth 1 gene, which encodes a RLCK VII-subfamily protein kinase, results in abnormal elongation growth following organ differentiation in Arabidopsis (Muto et al. 2004). The RLCK VII subfamily member Esi47 protein of wheat has been identified as an "early salt inducible" gene product with a role in the transduction of hormonal (gibberellin) signals (Shen et al. 2001).

A RLCKs, BIK1 (Botrytis Induced Kinase 1), originally identified as a component in plant defense against necrotrophic fungal pathogens (Veronese et al. 2006) it was recently showed to associate with flagellin receptor complex (FLS2/BAK1, two LRR-RLK) to initiate innate immunity (Lu et al. 2010).

Recently it was shown that NtNAK, a RLCK from class VII is an osmo-stress responsive protein kinase and phosphorylates a NtCK25 protein which is similar to human nuclear body protein SP110 involved in DNA/protein binding regulation (Kodama et al. 2009).

In Arabidopsis, BSK1 mediate brassinosteriods signaling, through the association with the brassionsteroids receptor Bri1, a LRR-RLK (Tang et al. 2008). BSK1 is phosphorylated by Bri1 but not by BAK1 which is know to interact with Bri1 in mediating the brassinosteroids signaling (Tang et al. 2008; Wang et al. 2005a, 2005b). 
However, little is known about the precise functions of RLCKs in the above processes. Based on these few examples, it is very likely, that they play major roles in the perception and transmission of external signals perceived by RLKs. Therefore, the transcriptional and posttranslational regulation of their activity can also be of high significance considering receptor signaling in plants.

Previously it was shown in our laboratory that the in vitro activity of two RLCKs (Class VI) of Medicago truncatula is active ROP GTPase-dependent (Dorjgotov et al. 2009). This observation, in agreement with that of Molendijk et al. (2008) about the in vitro and in vivo interaction of similar Arabidopsis RLCK kinases and ROPs, highlighted the possibility that RLCKs may serve as the "missing" ROP GTPase effectors in plants. Here we report on the characterization of the members of this RLCK family and provide further experimental evidences to support this hypothesis. 


\section{AIMS}

Our general aim was to characterize the members of the Arabidopsis thaliana RLCK Class VI family of protein kinases. This protein family was selected based on the previous observations that these plant-specific proteins may serve as ROP GTPase effector kinases (see above). Despite of their potential significance in ROP GTPase mediated signaling, hardly any functional information was available about the fourteen Arabidopsis RLCK Class VI members.

Therefore, we decided to carry out the following studies:

- in silico analysis of their primary structure

- gene expression profiling based on a gene-specific real-time quantitative PCR approach

- co-expression study of RLCKs and other ROP GTPase effectors and regulators based on in silico data analysis

- determination of RLCK-ROP GTPase interaction specificity in a yeast two-hybrid interaction matrix experiment

- investigation of in vitro phosphorylating activity and its dependence on the presence of ROP GTPases in active/inactive conformation

- generation of transgenic plants overexpressing or silencing RLCK VI members and their molecular and phenotypic characterization 


\section{MATERIAL AND METHODS}

\subsection{Sequence analysis}

cDNA and protein sequences were downloaded from the Arabidopsis Biological Resource Center (ABRC) at http://www.arabidopsis.org/abrc/. The accession numbers and the used nomenclature are shown in Figure 6. Sequence alignment was made using the ClustalW algorithm (Thompson et al. 1994). Phylogenetic analysis was carried out by the Phylip 3.67 program package (http://www.evolution.genetics.washington.edu/phylip.html). Protein sequence similarity was determined using the MatGAT 2.01 program (http://www.angelfire.com/nj2/arabidopsis/MatGAT.html).

Potential gene duplication events during the evolution of the RLCK VI family were analyzed by an online tool maintained by Ken Wolfe's laboratory at the Genetics Department, Trinity College, Dublin (http://www.wolfe.gen.tcd.ie/athal/dup). Domain information was collected from the PlantsP database (http://www.plantsp.genomics.purdue.edu/html/).

\subsection{Gene expression profiling}

\subsubsection{Plant material and growth conditions}

To analyze the gene expression of At RLCK_VI genes in different Arabidopsis organs, seeds from the Columbia ecotype were planted in the green house and plants were grown under 16 $\mathrm{h}$ light/8 h dark cycles conditions. Different organs, roots, rosette leaves, cauline leaves, inflorescence stems, flower buds, open flowers and green siliques were harvested from plants $\approx 60$ days after sowing. Arabidopsis thaliana ecotype Columbia exponentially growing cell suspension was also used. The culture was maintained by weekly subculturing in Murashige and Skoog (MS) medium supplemented with 30\%w/v sucrose, $240 \mathrm{ug} / 1$ 2,4-D and $14 \mathrm{ug} / 1$ kinetin (Fulop et al. 2005). All samples were frozen in liquid nitrogen immediately after harvesting and RNA was extracted.

For abiotic stress treatments, Arabidopsis seedlings were germinated on nylon meshes wetted by water in Petri dishes. Three-week-old seedlings were subjected to various stress/hormone treatments by moving the filters along with the plantlets to new dishes with water supplemented with the stress/hormone agents.

RNA samples were isolated from whole seedlings $6 \mathrm{~h}$ after the treatments. The treatments were as follows: polyethylene glycol (30\%), glucose (300 mM), sucrose ( $8 \%), \mathrm{NaCl}(300 \mathrm{mM})$, 
paraquat $(25 \mu \mathrm{M})$, abscisic acid $(100 \mu \mathrm{M})$, etephon $(10 \mathrm{mg} / \mathrm{l})$, salicylic acid $(10 \mathrm{mM})$. Seedlings were also transferred to pure water as control. Cold $\left(4^{\circ} \mathrm{C}\right)$ treatment as well as the corresponding control $\left(22^{\circ} \mathrm{C}\right)$ was carried out without transfer to new media. All the treatments were repeated three times.

\subsubsection{Nucleic acid isolation}

Total RNA was extracted from the frozen plant material using the acidic phenol/guanidium method (Chomczynski and Sacchi 1987). To avoid genomic DNA contamination, each RNA preparation was treated with RNAse-free DNAse according to the manufacturer's instructions (Sigma, St-Louis, USA). RNA was then quantified measuring absorbance at $260 \mathrm{~nm}$ using a spectrophotometer (NanoDrop Technologies, Wilmington, USA) and loaded on a denaturing agarose gel to check concentration and integrity.

Genomic DNA has been extracted from cultured Arabidopsis thaliana var. Columbia cells using the modified CTAB method (Aldrich and Cullis 1993) and was treated with RNase (Sigma).

\subsection{3 cDNA synthesis}

A measure of 2,5 $\mu \mathrm{g}$ total RNA was reverse transcribed using oligo dT primers and reverse transcriptase (Revert-Aid M-MuLV, Fermentas, Vilnius, Lithuania) according to the manufacturer's instructions. The cDNAs were diluted to $200 \mu \mathrm{l}$ with sterile $\mathrm{H}_{2} \mathrm{O}$.

\subsubsection{Real-time quantitative RT-PCR (qRT-PCR)}

Appropriate primer pairs allowing the differential identification of the 14 members of the Arabidopsis RLCK VI subfamily (Table 3 ) have been designed by the Primer Express software (Applied Biosystems, Foster City, USA). Real-time quantitative RT-PCR was assayed on an ABI Prism 7700 sequence detection system and the accumulation of PCR products was measured in real time as the increase in SYBR Green fluorescence using ROX (glycine conjugate of 5carboxy-X-rhodamine, succinimidyl ester) as a passive reference dye according to the manufacturer's (Applied Biosystems) instruction as described elsewhere in detail (Otvos et al. 2005; Szucs et al. 2006). Real time PCR reactions were carried out at least in duplicate with 6-6 $\mu 1$ cDNA samples in three biological repetitions. Two actin genes (actin2 and 8 transcripts coded by the At3g18780 and Atlg49240 genes, respectively, and detected together by the same primer pair; Charrier et al. 2002) were used as internal controls for data normalization (geNorm method, Vandesompele et al. 2002). 
Table 3: Genes and oligonucleotides used in the real-time RT-PCR experiments

\begin{tabular}{|c|c|}
\hline Sequences of the primers used & AGI code/name \\
\hline $\begin{array}{l}\text { 5'-CGGACATCCTGCCCTTGA-3' } \\
\text { 5'-GCTTTTCTCTCTAGCAACGGCTTA-3' }\end{array}$ & At3g05140/RLCK VI_A1 \\
\hline $\begin{array}{l}\text { 5'- GAGATATCAAAGCCGCAAACATTC -3' } \\
\text { 5'-TCGCAAGCCCAAAGTCACAT -3' }\end{array}$ & At5g35960/RLCK VI_A2 \\
\hline $\begin{array}{l}\text { 5'-CGGCTTCGATGGAGCGTAT-3' } \\
\text { 5'-CTTCGTGATTCCTCATTGATCACA-3' }\end{array}$ & At1g66460/RLCK VI_A3 \\
\hline $\begin{array}{l}\text { 5'- GCAACAACTTCATCGCATTGC-3' } \\
\text { 5'-TGGAAGGTCGACATAGAGAAGAAGAT -3' }\end{array}$ & At2g18890/RLCK VI_A4 \\
\hline $\begin{array}{l}\text { 5'- GGTTCAAATGGAGCGGATGAT-3' } \\
\text { 5'-GGTCTTCGGGACTCCTCATTG-3' }\end{array}$ & At5g37790/RLCK VI_A5 \\
\hline $\begin{array}{l}\text { 5'-ACAACAATAATGATGACCAAATGCA-3' } \\
\text { 5'-CTAGCTTGAGGACTACGTCGAATACA-3' }\end{array}$ & At1g77280/RLCK VI_A6 \\
\hline $\begin{array}{l}\text { 5'-GCTAAAATGGGCAAAGCTACAAGTT-3' } \\
\text { 5'-CCGCAATTTCTCGTCCTTGA-3' }\end{array}$ & At1g21590/RLCK VI_A7 \\
\hline $\begin{array}{l}\text { 5'-GCTGCCACGCTATGCATCA-3' } \\
\text { 5'-ATCTTTAGGACAAGGCCAATTTGC-3' }\end{array}$ & At5g63940/RLCK VI_B1 \\
\hline $\begin{array}{l}\text { 5'-GCAATGCGACCTGACATGACT-3' } \\
\text { 5'-TCACCTGCCTTCTGCTGTAGCT-3' }\end{array}$ & At5g10520/RLCK VI_B2 \\
\hline $\begin{array}{l}\text { 5'-CCTGCTTCTCGAGCTCATTACC-3' } \\
\text { 5'-AGCCCACATGACAATGCTATGTT-3' }\end{array}$ & At5g18910/RLCK VI_B3 \\
\hline $\begin{array}{l}\text { 5'-GACGAGGTTTACCCAAATTCGAA-3' } \\
\text { 5'-ACAGAGTCATTATCCTCCACGTCAA-3' }\end{array}$ & At2g16750/RLCK VI_B4 \\
\hline $\begin{array}{l}\text { 5'-GAACGTTTGGTTATCTAGCTCCAGAGT-3' } \\
\text { 5'-CGAATGCGTAGATGTCGGTTTT-3' }\end{array}$ & At5g57670/RLCK VI_B5 \\
\hline $\begin{array}{l}\text { 5'-ACGAAGCCGGGAAATGGA-3' } \\
\text { 5'-CGGGTAAACCTCGTCATCGA-3' }\end{array}$ & At4g35030/RLCK VI_B6 \\
\hline $\begin{array}{l}\text { 5'- CGAGATGAAACGAGTGATGCAA -3' } \\
\text { 5'-TCATGTCAGGACGCATAGTGGAT -3' }\end{array}$ & At5g65530/RLCK VI_B7 \\
\hline $\begin{array}{l}\text { 5'-GGTAACATTGTGCTCAGTGGTGG-3' } \\
\text { 5'-AACGACCTTAATCTTCATGCTGC-3' }\end{array}$ & $\begin{array}{l}\text { At3g18780/ACTIN_2 and } \\
\text { At1g49240/ACTIN_8 }\end{array}$ \\
\hline $\begin{array}{l}\text { 5'-ATTGGAAAATTGACCGGAATGT-3' } \\
\text { 5'-CGGTGAGATCAACAACTGAGACA-3' }\end{array}$ & At1g13440/GAPDH_2 \\
\hline
\end{tabular}

As further controls, the relative expression of one housekeeping gene coding for a glycerinaldehyde-3-phosphate dehydrogenase enzyme (GAPDH) (At1g13440; Czechowski et al. 2005) and one stress-induced gene RD29A (At5g52310; Yamaguchi-Shinozaki and Shinozaki 1993; for the used primers see Charrier et al. 2002) was also determined in the appropriate samples, respectively. In order to allow the comparison of the absolute gene expression levels of the 14 RLCK VI genes, genomic DNA was used as the standard. Genomic DNA was quantified and a range of three dilutions were prepared representing 200, 2.000 and 20.000 Arabidopsis 
genomes in $6 \mu \mathrm{l}$ of water, respectively. Steady state transcript levels were expressed as genome equivalents per ng initial RNA for each gene.

\subsubsection{In silico gene expression analysis}

Expression pattern of ROPs and their known/hypothesized regulators and effectors were analysed based on microarray data stored in the Genevestigator (Grennan 2006; Zimmermann et al. 2004) database and analyzed by a hierarchical clustering algorithm of the Genevestigator tool (www.genevestigator.com).

\subsection{Yeast two-hybrid assay}

In order to study protein-protein interaction the yeast two hybrid (Y2H) method was chosen. The $\mathrm{Y} 2 \mathrm{H}$ assay has the advantage of detection of interacting proteins in living yeast cells and it is fairly simple, rapid and inexpensive (avoids the costly protein purification and antibody development needed in traditional biochemical methods).

The base components of the yeast two-hybrid approach are transcription activator proteins. The yeast Gal4 transcription activator protein contains a DNA binding (aa 1-147) and an activation (aa 771-881) domains. In a notable finding, (Fields and Song, 1989) determined that the two separate and independent Gal4 domains, which lack function alone, could be synthesized as chimeric proteins with each Gal4 domain fused to one of two interacting partner proteins (X and $\mathrm{Y}$ ). The interaction between the partner proteins ( $\mathrm{X}$ and $\mathrm{Y}$ ) brings the separate activation domain (AD) into close proximity to the DNA binding domain (BD), thereby reconstituting the function of the Ga14 transcription activator protein and driving expression of a downstream reporter gene that enable growth on specific media. The interaction must take place in the nucleus for the subsequent activation of the reporter gene. For this purpose, non-nuclear proteins are targeted to the nucleus.

\subsubsection{Vector construction and mutant generation}

To test the interaction of AtRLCK kinases with AtROP GTPase, cDNA clones of 8 different Arabidopsis thaliana ROP GTPases, 10 Arabidopsis RLCK VI kinases (5 from group A- RLCK_A2, A3, A4, A6, A7 and 5 from group B- RLCK_B1, B2, B3, B5, B7) and three further kinases belonging to other RLCK families (IV, VII, IX) were inserted into yeast twohybrid vectors. The full length cDNAs of AtRLCK genes were obtained from ABRC (http://www.arabidopsis.org/abrc/) as cloned into the PUNI51 vector. The inserts were cloned 
from pUNI51 into the EcoRI-XhoI sites of the pENTRY4 (Invitrogen) vector. The insert was mobilized into the yeast two-hybrid activation-domain vector pAD-GAL4 2.1-DEST by performing a "Gateway" LR Clonase recombination reaction according to the advices of the manufacturer of the Gateway cloning system (Invitrogen, Carlsbad, California).

Rho GTPases only in the GTP-bound state can interact with effectors to elicit downstream signaling. Moreover, in eukaryotic cells these proteins undergo posttranslational isoprenylation on the cysteine residue within their C-terminal CAAX box and this prenylation is responsible for their association with appropriate membrane regions where they exert their function. Thus, for a successful yeast to hybrid assay mutations had to be introduced into these proteins. Therefore the constitutively active form (G15V) of 3 different Arabidopsis ROP GTPases (AtROP1, 4 and 6) and the localization (C-terminal GAAX) mutants of all 8 ROP GTPases were generated.

The constitutively active form of ROP GTPases was created by substitution of glycine with valine in position 15 of AtROPs which lead to inactivation of the intrinsic GTPase activity, thereby keeping the mutant protein permanently in its active, GTP-bound state. The CA (constitutive active mutant) forms of the AtROPs GTPase have been constructed by polymerase chain reaction (PCR) using oligonucleotide primers carrying the appropriate nucleotide changes (Table 4). The cloning strategy was the same in all cases: two separate PCR reactions were carried out using one of the two overlapping primers (AtROP-CA-fw/rev primers) carrying the appropriate mutation together with the appropriate primer planned either to the start or to the end of the cDNA (AtROP-fw/rev). The PCR products representing the overlapping 5' and 3' parts of the mutated cDNA were purified, mixed and the whole cDNA was amplified by the full AtROP$\mathrm{fw} / \mathrm{rev}$ primers that contain appropriate restriction sites to facilitate further cloning.

The non-prenylated (localization, L) variant of AtROPs was created by substitution of cysteine with glutamine in the CAAX box. For this, a modified reverse primer was used (Table 4). The primers for amplification of full sequence were extended by appropriate restriction sites to facilitate further cloning (Table 4). The fragment was then inserted at the EcoRI-SalI sites of pBD-GAL4 2.1. The mutation site was confirmed by sequencing. The Y2H vectors were purchased from Stratagene (La Jolla, California, United States). 
Table 4: Oligonucleotides used in the generation of constitutive active form (CA) and localization mutants (L) of AtROPs

\begin{tabular}{|c|c|}
\hline Sequences of the primers used & Primer name \\
\hline 5'-GCAGAATTCATGAGCGCTTCGAGGTTCGTAA-3’ & AtROP1-EcoRI-fw \\
\hline $5^{3}$-GCAGTCGACTCATAGAATGGATCCTGCCTTCTG-3's & AtROP1-SalI(L)-rev \\
\hline 5'-CGTGACGGTTGGCGATGTAGCTGTCGGAAA-3’ & AtROP1_CA BtgZI-fw \\
\hline 5'-TTTCCGACAGCTACATCGCCAACCGTCACG-3's & AtROP1_CA BtgZI-rev \\
\hline $5^{\prime}$-GCGGAATTCATGGCGTCAAGGTTTATA-3 & AtROP2-EcoRI-fw \\
\hline 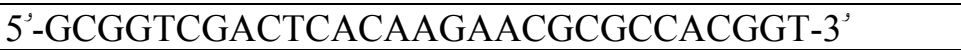 & AtROP2-SalI (L)-rev \\
\hline 5'-GCGGAATTCATGAGTGCTTCGAGGTTTAT-3's & AtROP4-EcoRI-fw \\
\hline 5'-GCGGTCGACTCACAAGAACACGCCGCGGT-3’ & AtROP4-SalI(L)-rev \\
\hline 5'-TGTCACCGTCGGGGATGTTGCCGTCGGAAA-3' & AtROP4_CA woBtgZI-fw \\
\hline $5^{\prime}$-TTTCCGACGGCAACATCCCCGACGGTGACA-3 ${ }^{\prime}$ & AtROP4_CA woBtgZI-rev \\
\hline 5'-GCGGAATTCATGAGTGCTTCAAGGTTTAT-3’s & AtROP6-EcoRI-fw \\
\hline 5'-GCGGTCGACTCAGAGTATAGAACCACCTT-3’ & AtROP6-SalI(L)-rev \\
\hline $5^{\prime}$-TGTCACTGTCGGAGACGTTGCTGTTGGAAA-3’ & AtROP6_CA BsmBI-fw \\
\hline 5'-TTTCCAACAGCAACGTCTCCGACAGTGACA-3' $^{\prime}$ & AtROP6_CA BsmBI-rev \\
\hline 5'-GCAGAATTCATGTCAGCTTCAATGGCTGC-3 & AtROP8-EcoRI-fw \\
\hline 5'-GCAGTCGACTCAAAGAACATGGCCTAAACCGA-3’ & AtROP8-SalI(L)-rev \\
\hline $5^{\prime}$-GCAGAATTCATGAGTGCTTCGAAGTTCAT-3 & AtROP9-EcoRI-fw \\
\hline $5^{\prime}$-GCAGTCGACTTAAGCAGCGGTACCACCTCCACA-3 ${ }^{\prime}$ & AtROP9-SalI(L)-rev \\
\hline 5'-GCGGAATTCATGGCTTCGAGTGCTTCAAA-3 & AtROP10-EcoRI-fw \\
\hline 5'-GCGGTCGACTCAATTCTTCCCACCCAG-3 ${ }^{\circ}$ & AtROP10-SalI(L)-rev \\
\hline $5^{\prime}$-GCGGAATTCATGGCTTCAAGTGCTTCAAA-3's & AtROP11-EcoRI-fw \\
\hline 5'-GCGGTCGACTCAATGCCGAGTCACTATCC-3' & AtROP11-SalI(L)-rev \\
\hline
\end{tabular}

\subsubsection{Yeast transformation and selection}

Yeast transformation was done as described in (Agatep et al. 1998) and the Saccharomyces cerevisiae yeast strain PJ69-4a was used as host.

The genotype of yeast strains is: MATa, trp1-901, leu2-3,-112, ura3-52, his3-200, gal4A, gal804, GAL2-ADE2, LYS2:GAL1-HIS3, met2:GAL7-lacZ.

The transformants were grown on appropriate drop out media to monitor transformation efficiency as well as the activation of the HIS3 (in the presence of $1 \mathrm{mM} 3$-aminotriazol) and/or the ADE2 reporter genes. Protein-protein interactions were detected in double (-Trp,-Leu), triple (-Trp,-Leu,-His) and quadruple (-Trp,-Leu,-His,-Ade) selection plates.

Both positive and negative controls were included in the experiment, like $\operatorname{RepA}(\mathrm{C} 1) / \mathrm{pGAD} 424$ and ZmRB/pGBT9 constructs (described by Dr. Horvath Gabor and its collaborators) and empty pAD-GAL4 and pBD-GAL4 vectors respectively. 


\subsection{Biochemical characterization of AtROPs and AtRLCK VI}

\subsubsection{Expression of AtROPs and AtRLCKs in Escherichia coli}

Full length cDNA sequences of ROP GTPases were cloned into the vectors pMAL2c, pBAD, pTRC-HisC and pET26b, while the RLCK-full length coding cDNAs were inserted into pET28c (for details see Table 5).

Table 5: Vectors and genes used for the expression of recombinant proteins in Escherichia coli

\begin{tabular}{|c|c|c|c|c|}
\hline Vectors & Genes & Characteristics of vector & $\begin{array}{c}\text { Induction } \\
\text { agent }\end{array}$ & $\begin{array}{c}\text { Elution } \\
\text { agent }\end{array}$ \\
\hline \hline $\begin{array}{c}\text { pMAL2c } \\
\text { pMAL Dest }\end{array}$ & AtROP1; AtROP1_CA & $\begin{array}{c}\text { N-terminal maltose } \\
\text { binding protein, MBP; } \\
\text { New England Biolabs }\end{array}$ & IPTG & maltose \\
\hline pBAD & AtROP1 & $\begin{array}{c}\text { N-terminal 6xHIS tag, } \\
\text { Invitrogen }\end{array}$ & L-arabinose & imidazole \\
\hline pTRC-HisC & AtROP1_CA & $\begin{array}{c}\text { N-terminal 6xHIS tag; } \\
\text { Invitrogen }\end{array}$ & IPTG & imidazole \\
\hline pET26b & AtROP1; AtROP6 & $\begin{array}{c}\text { C-terminal 6xHIS, } \\
\text { Novagen }\end{array}$ & IPTG & imidazole \\
\hline pET28c & $\begin{array}{c}\text { AtRLCK VI_A2, A3, } \\
\text { A6, A7, B3, B5, VII_1 }\end{array}$ & $\begin{array}{c}\text { N-terminal 6xHIS, } \\
\text { Novagen }\end{array}$ & IPTG & imidazole \\
\hline
\end{tabular}

Protein production was achieved in the Escherichia coli strain Rosetta ${ }^{\mathrm{TM}}$ [BL21 (DE3)/(pLysS)] (Novagen). For this, one isolated colony was inoculated in 20ml LB medium (10 $\mathrm{g} / \mathrm{l} \mathrm{NaCl}, 10 \mathrm{~g} / 1$ Tryptone, $5 \mathrm{~g} / \mathrm{l}$ yeast extract) and grown $12-14 \mathrm{~h}$ at $37^{\circ} \mathrm{C}$ with constant shaking (250 rpm). The culture was then diluted $20 \mathrm{x}$ to $200 \mathrm{ml}$ culture and grown for an additional $3 \mathrm{~h}$ at $37^{\circ} \mathrm{C}$.

Purification of MBP-tagged proteins using amylose resin was carried out as recommended by New England Biolabs. Expression of MBP-fused proteins was induced by addition of $1 \mathrm{mM}$ isopropyl-1- thio-/3-Dgalactopyranoside (IPTG) to the culture and incubation for additional $3 \mathrm{~h}$ at $37^{\circ} \mathrm{C}$ with continuous shaking. The following buffers were used: for binding - MBP buffer (20 mM Tris pH=7,4, $200 \mathrm{mM} \mathrm{NaCl,} 1 \mathrm{mM}$ EDTA, $10 \mathrm{mM}$ 2-mercaptoethanol); for elution - $10 \mathrm{mM}$ maltose was added to MBP buffer. 
HIS tagged protein purification using HisSelect Sepharose (Sigma) has been modified. The expression of recombinant proteins was induced by addition of $1 \mathrm{mM}$ isopropyl-1- thio-/3Dgalactopyranoside (IPTG) to the culture followed by growth for 45 minutes at $30^{\circ} \mathrm{C}$ in the case of kinases and for $1,5 \mathrm{~h}$ at $37^{\circ} \mathrm{C}$ for GTPases. If $\mathrm{pBAD}$ vector was used for the expression of the protein, the induction was done by addition of $0.2 \% \mathrm{~L}$-arabinose for $2 \mathrm{~h}$ at $37^{\circ} \mathrm{C}$ under continuous shaking conditions. The buffers used were the following: for binding - $20 \mathrm{mM}$ Tris- $\mathrm{HCl} \mathrm{pH}=8,0$, $200 \mathrm{mM} \mathrm{NaCl}, 5 \mathrm{mM}$ imidazole, $10 \mathrm{mM}$-mercaptoethanol and 0.1\% Triton 100x; for washing $10 \mathrm{mM}$ imidazole and $0,4 \%$ Triton $100 \mathrm{x}$ were added to the binding buffer; for elution - $20 \mathrm{mM}$ Tris- $\mathrm{HCl} \mathrm{pH}=8,0,200 \mathrm{mM} \mathrm{NaCl}, 150 \mathrm{mM}$ imidazole.

Eluted proteins were concentrated by CentriCon filters (Millipore) and stored in 50\% glycerol at $-20^{\circ} \mathrm{C}$.

Western blotting was carried out using standard procedures. Peroxidase-conjugated antipoly histidine and anti-rabbit IgG antibodies were purchased from Sigma, while the anti-maltosebinding protein serum from New England Biolabs. Immunoreactive bands were visualized by chemiluminescence using Lumi-Light Western Blotting Substrate (Roche) and X-ray films (Kodak).

\subsubsection{In vitro protein interaction}

The pull-down assay was used to determine the physical interaction between the proteins of interest. The principle of the method is that the tagged bait (expressed in E.coli) is immobilized on a resin and subsequently "pulls down" tagged target protein (prey) from lysates (of E. coli expressing the protein of interest).

In this procedure, AtROP1 tagged with MBP (maltose-binding protein) and coupled to amylose resin were used as an affinity matrix for the co-purification of HIS-tagged AtRLCK kinases expressed in E. coli.

The experiment was carried out as follows in details. MBP-tagged AtROP1 and MBP alone (as negative control) were expressed in E. coli and bounded to amylose resin. Further, the AtROP1 bounded to resin were incubated for 20 minutes at RT in an EDTA buffer $(20 \mathrm{mM}$ Tris (pH 7.5), 1 mM DTT, $10 \mathrm{mM}$ EDTA, $50 \mathrm{mM} \mathrm{NaCl}, 5 \%$ (v/v) glycerol and 0,1\% (v/v) Triton X100) to prepare the $G$ protein in a nucleotide-depleted state. The beads were then separated into three equal aliquots. One aliquot was stored on ice while the other two aliquots were used to prepare the GDP- and GTP-bound G proteins. These were incubated in nucleotid-binding buffer 
(20 mM Tris (pH 7,5), $1 \mathrm{mM}$ DTT, $10 \mathrm{mM} \mathrm{MgCl} 2,50 \mathrm{mM} \mathrm{NaCl}, 5 \%$ (v/v) glycerol and 0,1\% (v/v) Triton X-100) containing either $200 \mu \mathrm{M}$ GDP or $20 \mu \mathrm{M}$ GTP $\gamma \mathrm{S}$ for 25 minutes at room temperature. The E. coli cell lysates expressing HIS-tagged AtRLCK were added to the MBPtagged AtROP1 samples (nucleotide-depleted, GTP-bounded and GDP-bounded) and to MBPbound resin and the amylose resin alone for negative control. The mixture was incubated for 2 hours at $4^{\circ}$. After incubation time, the beads were washed and resuspended in 1XSDS loading buffer and subjected to SDS-PAGE. For Western blotting, a peroxidase-conjugated anti-poly histidine (Sigma) and an anti-maltose-binding protein serum from New England Biolabs were used followed by an anti-rabbit IgG antibody purchased from Sigma. Immunoreactive bands were visualized by chemiluminescence using Lumi-Light Western Blotting Substrate (Roche) and Xray films (Kodak).

\subsubsection{Protein kinase assay}

For kinase activity measurement, 2 pmol purified kinase, 5 pmol purified small GTP binding protein and $0,25 \mu \mathrm{g} / \mu \mathrm{l}$ myelin basic protein were combined in $20 \mu \mathrm{l}$ kinase reaction buffer (20 mM Tris-HCl pH 7,6, $5 \mathrm{mM} \mathrm{MgCl} 2,1 \mathrm{mM} \mathrm{DTT}, 10 \mathrm{~mm} \mathrm{ATP,} \mathrm{0,} 2 \mathrm{MBq}[\gamma-32 \mathrm{P}]$ ATP) and kept for 30 minutes at room temperature. Termination of the assay was carried out by adding $5 \mu$ of $5 \times$ SDS sample buffer to the reaction. The protein samples were separated by SDS polyacrylamide gel and the dried gels were analysed by a Phosphorimager 445 SI station.

\subsection{Transgenic plant generation and characterization}

\subsubsection{Biological material and vector preparation}

The Columbia ecotype of Arabidopsis thaliana was used for the generation of transgenic plants. The Agrobacterium tumefaciens GV3101/pMP90 strain carrying the appropriate binary vector was used to transfer the chimeric constructs into the plant cells.

For the transformation of Arabidopsis thaliana Columbia, three different binary vector systems were used. Two vectors for over-expressing the gene of interest (pJCGLOX and pER8) and one RNAi vector (pER8-RNAi) to knock down the gene of interest.

The pJCGLOX vector (for details see http://www.psb.ugent.be/gateway) is a derivative of the pCAMBIA 2200 vector (for details, see http://www.cambia.org) (Figure 4). 


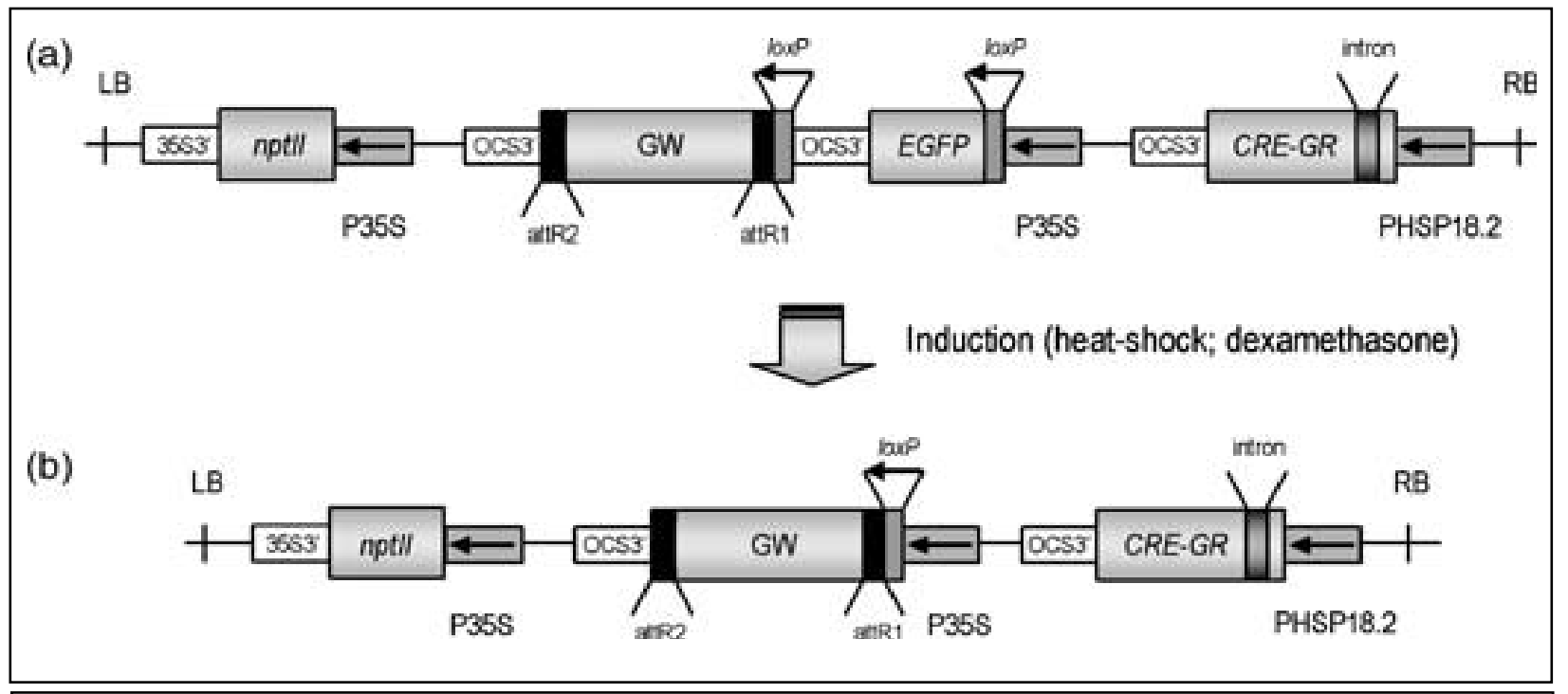

Figure 4: T-DNA region of pJCGLOX destination vector (Jerome et al. 2004). (a) The kanamicinresistant element consists in the coding sequence of the neomycin phosphotransferase II (nptII) gene, flanked by the CaMV35S promoter (P35S) and terminator (35S3') sequences. The coding sequence of the CRE recombinase interrupted by an intron and fused to the GR sequence is under the control of the promoter of the Arabidopsis HSP18.2 gene (PHSP 18.2) and terminated by the OCS3' sequence. The $E G F P$ coding sequence ended by the OCS3' sequence is flanked by two loxP sites, and is under control of the P35S. Downstream of the second loxP site, the GATEWAY ${ }^{\mathrm{TM}}$ recombination site (GW:attR1, CmR, $c c d B$, attR2, rfA of the GATEWAY ${ }^{\mathrm{TM}}$ vector conversion system; Invitrogen) is terminated by the OCS3' sequence. LB and RB, T-DNA left and right border, respectively. (b) Excision of the EGFP coding sequence flanked by the two loxP sites by the induced ligand-inducible CRE-GR recombinase, thereby generating constitutive expression of the gene of interest under control of the P35S.

XVE expression vectors pER8-GW and pER8-RNAi-GW (see Zuo et al. 2000) were used. The XVE chimeric transcription factor, expression of which is controlled by the strong constitutive G10-90 promoter is transcriptionally inactive in the absence of an inducer. When activated by the chemical inducer ${ }^{17} \beta$-estradiol, a mammalian hormone that has no detectable effects on plant growth and development, XVE specifically binds to a LexA operator sequence, thereby initiating transcription from $\mathrm{O}^{\mathrm{LexA}}-46$ promoter. The DNA fragment is cloned downstream from the $\mathrm{O}^{\mathrm{LexA}}-46$ promoter, thus allowing expression of the target gene in a ${ }^{17} \beta$ estradiol dependent manner (Figure 5).

For RNAi-mediated gene silencing, targeted regions of the genes were cloned in sense and antisense orientation, separated by an intron, into the binary vector pER8 (Figure 5b), creating the estrogen-inducible, hairpin RNA-forming cassettes. 


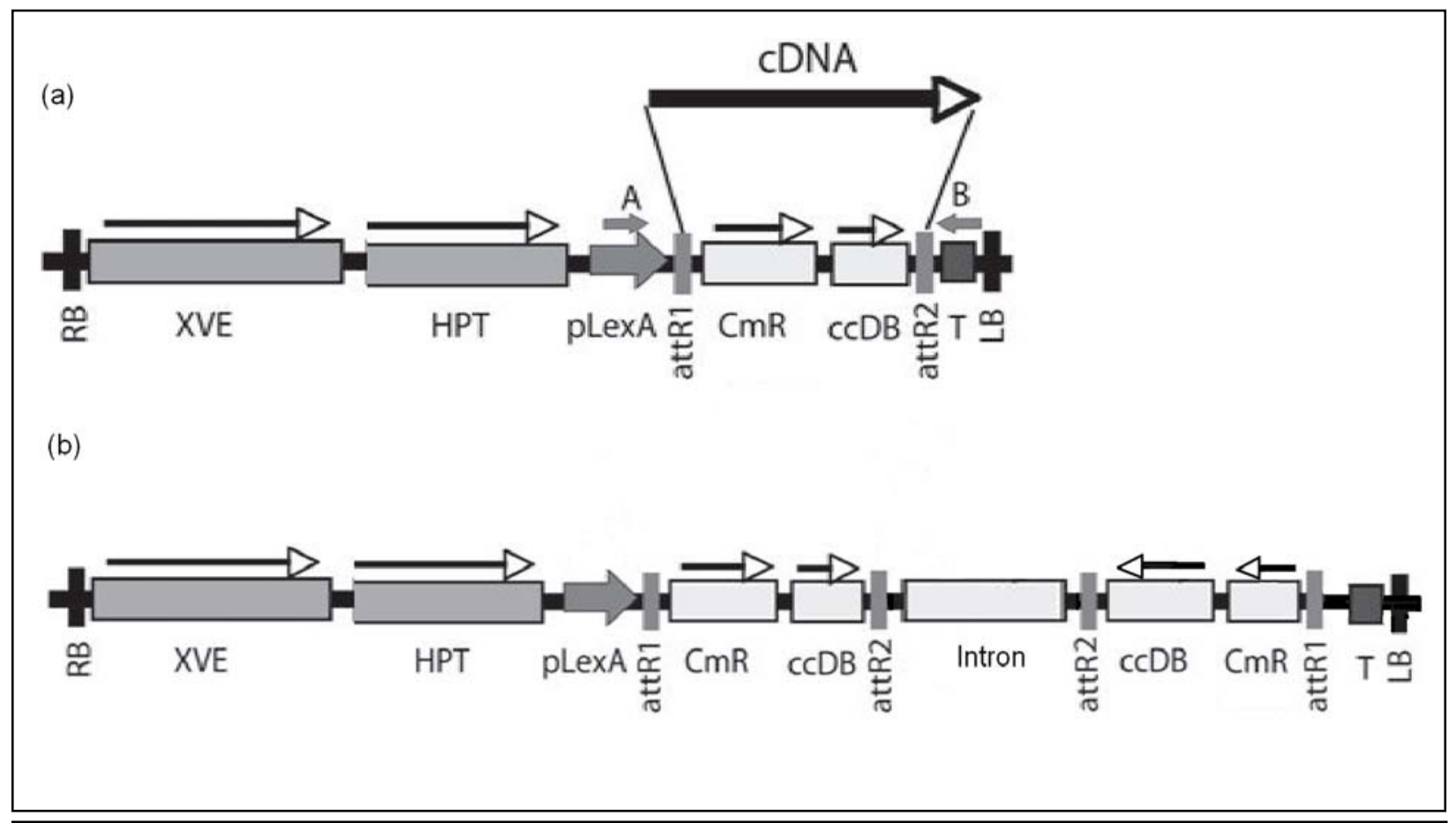

Figure 5: Schematic illustration of the T-DNA region of the pER8 vectors (Zuo et al. 2000). (a) pER8-GW vector, (b) pER8-RNAi-GW vector. XVE: chimaeric XVE fusion gene, encoding the chimaeric transcription activator for the pLexA promoter, HPT: hygromycin phosphotransferase gene, pLexA: LexA operator fused to a minimal promoter of Cauliflower Mosaic Virus 35S gene, attR1 and attR2: Gateway recombination sites, CmR: Chloramphenicol resistance gene, ccdB: suicide marker for bacterial contraselection, T: RUBISCO rbcsS3A polyA sequence; RB and LB: T-DNA left and right border sequences, respectively. cDNA: randomly inserted cDNA clone; A and B: positions of T-DNA specific PCR primers used for amplification of inserted cDNAs. Intron cassette - Intron 1 of Arabidopsis thaliana WRKY gene.

The pJCGLox vectors containing the full length cDNA of RLCK-A2 and AtROP6 wt and AtROP6-CA (constitutively active form) were obtained by performing a LR Clonase recombination reaction between the pJCGLox destination vector and the pENTRY4 vector containing the respective insert.

The full cDNA sequence of AtRLCK-A2 was cloned to pER8 vector by LR clonase recombination reaction using the pENTRY4 donor vector containing the insert. To obtain the RNAi clones, a LR Clonase reaction between the pER8-RNAi vector and pENTRY207 vector containing short gene-specific sequences (GSTs) of AtRLCK-A2, was performed. The pENTRY207 vectors containing the short GST sequences of the kinase of interest were obtained from the "AGRIKOLA" consortium (www.agrikola.org) through the Arabidopsis Biological Resource Center (ABRC; http://www.arabidopsis.org). 


\subsubsection{Transformation of Agrobacterium tumefacines}

The obtained constructs (pJCGLOX, pER8 and pER8-RNAi vectors containing the inserts) were mobilized into Agrobacterium tumefaciens (strain GV3101/pMP90) by triparental mating using a conjugation helper plasmid HB101/pRK2013. The Agrobacterium conjugants were selected on LB media containing $100 \mathrm{mg} / \mathrm{l}$ kanamycin and $100 \mathrm{mg} / \mathrm{l} \mathrm{rifampicin,} 25 \mathrm{mg} / \mathrm{l}$ gentamicin and $100 \mathrm{mg} / \mathrm{l}$ spectinomycin or $100 \mathrm{mg} / \mathrm{l}$ chloramphenciol plates. The spectinomycin was used for the selection of the pER8 vectors and chloramphenciol for the selection of the pJCG vector. Selected conjugants were screened by restriction digestion and PCR using insert specific primers. The positive conjugants containing the vector of interest were used for Arabidopsis transformation.

\subsubsection{Transformation of Arabidopsis thaliana}

The Columbia ecotype of Arabidopsis thaliana was used for the generation of transgenic plants. Plants were grown in standard potting mix at a density of 10-15 plants per pot, under $16 \mathrm{~h}$ light $/ 8 \mathrm{~h}$ dark cycles conditions. The first flowering shoots were removed as soon as they emerged. Plants were ready for transformation, when the secondary inflorescence shoot were $\sim 8 \mathrm{~cm}$ tall.

The Agrobacterium tumefaciens strains, carrying the appropriate plant transformation vector, were inoculated in liquid YEB media $(0,5 \mathrm{~g}$ beef extract, $0,1 \mathrm{~g}$ yeast extract, $0,5 \mathrm{~g}$ peptone, 0,5g sucrose, $30 \mathrm{mg} \mathrm{MgSO}_{4} \times 7 \mathrm{H}_{2} \mathrm{O}$ ) containing the appropriate antibiotics and were grown for $24 \mathrm{~h}$ at $28^{\circ} \mathrm{C}$. Before transformation, the cultures were resuspended in $1 \times B 5$ medium pH5,7, supplemented with $5 \%$ sucrose and $0,02 \%$ SILWET L-77. The next step was the infiltration which was done by immersing the inflorescences in the suspension of Agrobacterium tumefaciens for $\sim 30$ seconds. The plants were kept for $24 \mathrm{~h}$ in dark (covered by aluminum folia) than the plants were allowed to set seed in the greenhouse under normal conditions.

\subsubsection{Plant selection and molecular characterization}

Arabidopsis $\mathrm{T}_{0}$ seeds obtained after floral dip transformation were surface sterilized by immersion in $70 \%(\mathrm{v} / \mathrm{v})$ ethanol for 1 min, followed by immersion in $30 \%(\mathrm{v} / \mathrm{v})$ sodium hypochlorite solution for $10 \mathrm{~min}$. Seeds were then washed four times with sterile distilled water and sown onto Murashige and Skoog (MS) medium (4,4 g/1 MS salt including vitamins, 30\% sucorse, $\mathrm{pH}$ 5,8, $6 \mathrm{~g} / \mathrm{l}$ agar) containing $250 \mathrm{mg} / \mathrm{l}$ Cefotaxim and $50 \mathrm{mg} / \mathrm{l}$ kanamycin, or $15 \mathrm{mg} / \mathrm{l}$ hygromycin. After a two-days stratification period, plates were transferred to growth chamber at 
$22^{\circ} \mathrm{C}$. After $\sim 3$ weeks the kanamycin and hygromycin resistant transformants were transferred to the green house into pots containing a 3:1 mixture of standard potting compost and vermiculite, under short day condition ( $8 \mathrm{~h}$ light/16h dark) for 2-3 weeks. The plants were transferred to long day condition (16h light/8h dark) to start flowering.

For checking the inducibility of transgene expression, in the case of plants transformed with pJCGLOX vectors, leaves were collected from the transformants grown in the green house and incubated for $2 \mathrm{~h}$ at $37^{\circ} \mathrm{C}$ and then transferred to the growth chamber at $22^{\circ} \mathrm{C}$ for $24 \mathrm{~h}$. The leaves were kept all this time in small Petri dishes on a wet filter paper to avoid drying. In the experiment a non-treated control was included as well as leaves from wild type Arabidopsis plants. After 24h, the leaves were collected and frozen in liquid nitrogen for RNA extraction as described earlier.

In the case of plants transformed with pER8 or pER8-RNAi vectors, leaves were collected from the green house plants and sprayed with $10 \mu \mathrm{M}{ }^{17} \beta$-estradiol. A non-treated control as well as leaves from wild type Arabidopsis plants were included in the experiment. Samples were collected at 6 and 24h after estradiol treatment and frozen in liquid nitrogen.

Total RNA extraction and cDNA synthesis were performed as described above. Semiquantitative RT-PCR and qRT-PCR were used to quantify gene expression in the transgenic lines.

Semi-quantitative PCR reactions were performed in $20 \mu \mathrm{l}$ volume, using $2 \mu \mathrm{l}$ cDNA template and Dream $\mathrm{Taq}^{\mathrm{TM}}$ polymerase (Fermentas) employing the following protocol: denaturation 1 cycle at $94^{\circ} \mathrm{C} / 2 \mathrm{~min}$., 30 cycles of $94^{\circ} \mathrm{C} / 30 \mathrm{sec}$., $55^{\circ} \mathrm{C} / 30 \mathrm{sec}$., and $72^{\circ} \mathrm{C} / 2 \mathrm{~min}$. and 1 cycle $72^{\circ} \mathrm{C}$ for $5 \mathrm{~min}$. Gene specific primers were used for detection of AtRLCK VI_A2 transcripts (A2-EcoRI full-fw: GCAGAATTCATGAAGTACATTCGAAGCAA and A2-XhoI full-rev: GCACTCGAGGCGACTACGATGCGAAGAAC). A control RT-PCR reaction was performed for every transgenic line using primers that amplify the 18S rRNA (18s-fw: AGGCGCGCAAATTACCCAAT and 18s-rev: TCTTCAAAGTAACAGCGCCGGA). The amplified fragments were separated on an agarose gel.

qRT-PCR reactions were performed as described above, using the following primers: for detection of AtRLCK VI_A2 transcription: 5'- GAGATATCAAAGCCGCAAACATTC-3'/5'TCGCAAGCCCAAAGTCACAT -3'; and for the reference gene (GAPDH-2): 5'ATTGGAAAATTGACCGGAATGT-3'/5'-CGGTGAGATCAACAACTGAGACA-3'. 


\subsubsection{Pollen germination and microscopic visualization}

Young flowers from Arabidopsis thaliana var. Columbia transgenic (homozygous plants) and non-transgenic plants were used for the examination of pollen tube phenotype. The collected flowers were placed into moisture chambers constructed from Petri dishes, and incubated for 30 minutes at $22^{\circ} \mathrm{C}$ followed by 30 minutes at $30^{\circ} \mathrm{C}$ to increase the germination rate of the pollen grains. The pollen was spread on the surface of an agarose pad $(0,5 \times 1 \mathrm{~cm})$ that was drawn on a glass microscope slide. The agarose medium $\left(0,01 \%\right.$ boric acid, $5 \mathrm{mM} \mathrm{CaCl}_{2}, 5 \mathrm{mM} \mathrm{KCl}, 1 \mathrm{mM}$ $\mathrm{MgSO}_{4}, 10 \%$ sucrose, $\mathrm{pH} 7,5$ and 1,5\% low-melting agarose (Boavida 2007)) was supplemented with $10 \mu \mathrm{M}{ }^{17} \beta$-estradiol or, as a control, the pollengrains were germinated on normal medium. The slides were placed inside a moisture incubation chamber (Petri dishes) to avoid dehydration of the pollen and incubated for $24 \mathrm{~h}$ at $22^{\circ} \mathrm{C}$ in dark. Pollen was examined using Nikon Eclipse TE 300 Epifluorescence microscope with a SPOT RT II color CCD camera.

\subsubsection{Trichome isolation and morphological analysis}

The trichomes were isolated from mature leaves of 3-weeks-old seedlings. For this, $\mathrm{T}_{1}$ seeds from transgenic (over-expressing and silencing) as well as non-transgenic Arabidopsis plants were germinated on Murashige and Skoog (MS) medium (4,4 g/1 MS salts including vitamins, $30 \mathrm{~g} / 1$ sucrose, $\mathrm{pH} 5,8$ and $6 \mathrm{~g} / 1$ agar) supplemented with $10 \mu \mathrm{M}{ }^{17} \beta$-estradiol. The seedlings were sprayed with the same concentration of ${ }^{17} \beta$-estradiol once at 2 days. As control, seeds from the same plants were germinated on normal MS media. RNAi lines were kept at $25^{\circ} \mathrm{C}$.

After 3 weeks, when the true leaves were completely formed, the trichomes were isolated for further analysis. The mature leaves were fixed in 50\% (v/v) ethanol and 5\% (v/v) acetic acid for $1 \mathrm{~h}$ at room temperature. Following fixation, leaves were washed three times in PEMT (25 mM PIPES, 2,5 mM EGTA, 0,5 mM MgSO $4,0,05 \%$ Triton X-100, $\mathrm{pH} 7,2$ ) for 10 minutes to remove fixative. Leaves were transferred to new tubes containing PEMT supplemented with 50 $\mathrm{mM}$ EGTA and vacuum infiltrated for 15 minutes and incubated $24 \mathrm{~h}$ at $4^{\circ} \mathrm{C}$. Leaves were transferred to Petri dishes and trichomes were removed by gentle rubbing using a paintbrush. Trichomes were washed three times with PBST (phosphate-buffered saline containing 0,05\% TritonX-100, $\mathrm{pH} 7,2)$ and stained with $0,05 \%(\mathrm{w} / \mathrm{v})$ Toluidine Blue supplemented with $0,05 \%$ Triton X-100 for $1 \mathrm{~h}$ and rinsed twice in PBST. The trichomes were visualized with a STEREOmicroscope. 


\section{RESULTS}

\subsection{Sequence analysis}

The aminoacid sequences of all members belonging to the RLCK Class VI protein family of Arabidopsis and three further kinase proteins that belongs to three other classes, (RLCK VIINAK; RLCK VIII-Pti1 and a MAPK-HPK1) were analyzed.

The family of class VI receptor-like cytoplasmic kinases (RLCK VI) of Arabidopsis consists of 14 members. Sequence comparison and phylogenetic analysis revealed that these proteins are highly homologous to each other, especially in the kinase domain, but are divergent from the related kinase families (Figure 6). It could also be determined that the 14 RLVK VI kinases form two groups with 7 members each. The RLCK VI family members have been designated accordingly as RLCK VI_A1-A7 and B1-B7 (see Figure 6 for details). Furthermore, within these groups, there are well recognizable pairs of sequences with high similarity to each other, possibly as the result of gene duplication events (see Figure 6).

The similarity at the kinase domain among the group A members range from $72,3 \%$ to 93,1\%. When the kinase domain of the group A kinases are compared to that of the RLCK VI_B members, the similarity range from $57,4 \%$ to $68,2 \%$. Comparison of RLCK VI kinases to representatives of other RLCK classes (class VII, VIII) and to an Arabidopsis MAPK indicated a range of similarity from $51,0 \%$ to $56,7 \%$ and from $39,4 \%$ to $45,7 \%$ respectively.

Despite of the considerable similarity of the kinase domain of all RLCK VI members, the overall length (ranging from 392 to 794 aminoacids) and domain composition of the individual proteins are divergent. Six of the RLCK VI kinases, five of them belonging to group B, contain the "universal stress protein" (UspA) signature.

In contrast, four members of RLCK VI group A have a N-terminal serine-rich region. As listed in Figure 6, there are few other regions exhibiting homology to protein domain database sequences in the RLCK VI kinases. The functional significance of these regions still needs to be verified. 


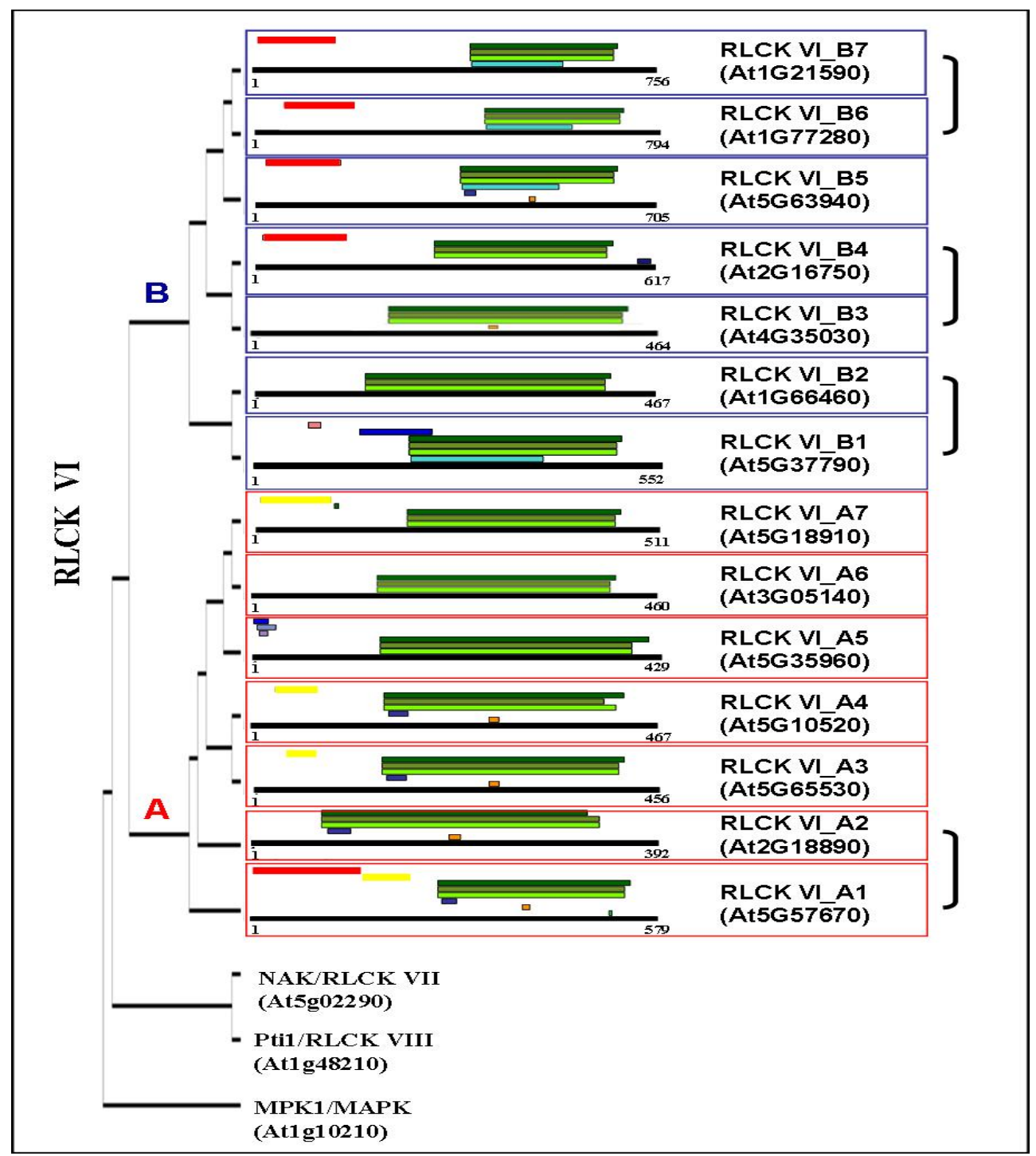

Universal stress protein family domain (PF00582)

Protein kinase catalytic domain profile (PS50011)

Protein kinase domain (PF00069)

Protein tyrosine kinase (PF07714 )

Aminoglycoside phosphotransferase (PF01636)

Protein kinase ATP-binding region signature

(PS00107)

$\mathrm{S} / \mathrm{T}$ protein kinases active-site signature (PS00108)

Serine-rich region profile (PS50324)

Glycine-rich region profile (PS50315)

SepZ (PF06066)

Serine proteases, trypsin family, histidine active site (PS00134)

Histidine-rich region profile (PS50316)

Prokaryotic membrane lipoprotein lipid attachment site (PS51257)

Potential transmembrane spanning (hydrophobic)

region

Prokaryotic membrane lipoprotein lipid

attachment site (PS00013)

Figure 6: Phylogenetic relationship and domain organization of Arabidopsis RLCK VI protein kinases. The protein sequences of the 14 RLCK VI kinases and 3 other Arabidopsis kinases have been compared. The cladogram on the left indicates the phylogenetic relationship of the investigated proteins. The RLCK VI kinases falls into two groups (A, B) and were designated accordingly. The hooks at the right indicate duplicated genes (paralogons) residing in wellrecognizable duplicated chromosome regions based on the search of paralogons in the Arabidopsis genome (http://www.wolfe.gen.tcd.ie/athal/dup) 


\subsection{Expression analysis}

\subsubsection{Absolute levels of the RLCKs VI transcripts}

The steady-state transcript level of the 14 AtRLCK VI genes was measured in total RNA extracted from cell suspension and seven different Arabidopsis organs, namely roots, rosette leaves, cauline leaves, inflorescence stems, flower buds, open flowers and siliques using the technique of real-time quantitative RT-PCR. In this study, genes coding for the cytoskeleton protein ACTIN were chosen as housekeeping reference genes. Although members of the Arabidopsis ACTIN gene family are differentially expressed (Huang et al. 1997), An et al. (1996) demonstrated that the two members, ACT2 and ACT 8, display complementary patterns of expression, making their combined expression profile quasiconstitutive. Thus the absolute transcript level of the 14 RLCK VI genes and the control At1g13440 (GAPDH) gene were normalized to ACTIN 2/8 gene expression. Furthermore, all the genes included in the experiment were tested in the same cDNA samples to allow direct comparison.

In order to allow the comparison of the absolute gene expression levels of the 14 RLCK VI genes, genomic DNA was used as the standard. Genomic DNA was quantified and a range of 3 dilutions was prepared representing 200, 2.000 and 20.000 Arabidopsis genomes in $6 \mu$ of water, respectively. Steady state transcript levels were expressed as genome equivalents per ng initial RNA for each gene.

Figure 7 illustrates the range of transcript levels of the investigated RLCK VI genes in the plant organs. It can be seen that the average expression of the RLCK VI genes was below 10.000 genome equivalent copies per ng total RNA (ge ng RNA ${ }^{-1}$ ). A control gene was included in the experiment and this was a gene coding for glyceraldehyde 3-phosphate dehydrogenase (GAPDH). The data shows that the average transcript level of the GAPDH gene was close to 200.000 ge. copies/ng RNA.

Despite the relatively low level of expression, all RLCK VI genes displayed a broad range in their transcript abundance in the various plant organs indicating the developmental regulation of their transcription RLCK VI_A6 and B7 showed the lowest expression level while RLCK VIB1 showed the highest level of expression. The average expression strength of the duplicated genes (paralogons) was similar, except for RLCK VI_B1 and B2. 


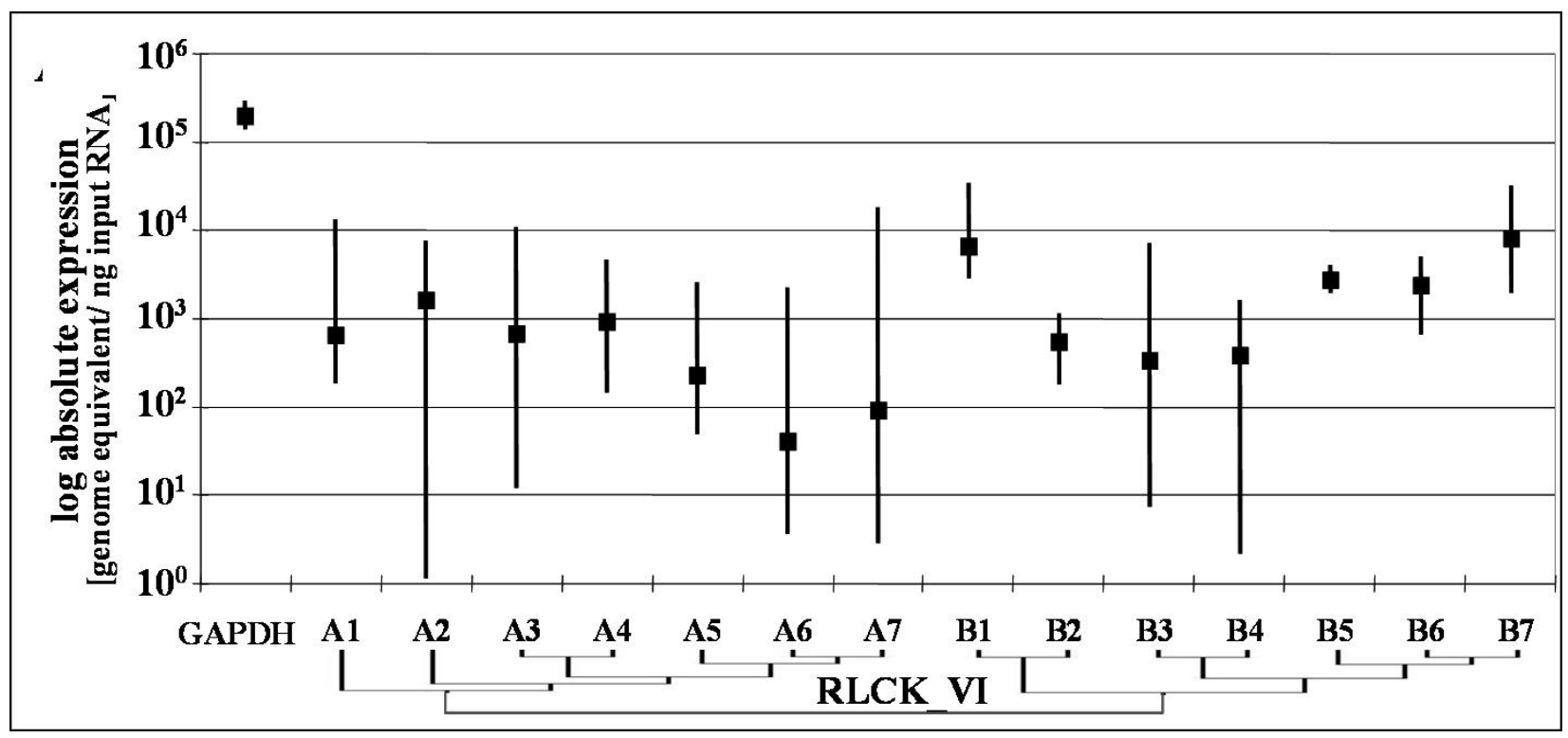

Figure 7: Range of the absolute steady-state transcript level for 14 RLCK VI genes and the control Atlg13440 (GAPDH) gene. Y axis is a $\log _{10}$ representation of the number of ge per nanogram of total RNA (ge ng $\mathrm{RNA}^{-1}$ ). The results are represented as a bar spreading from the highest to the lowest levels observed, with a square representing the mean transcript level. The cladogram below the histogram shows the phylogenetic relation of the genes.

\subsubsection{Relative expression profile of the 14 RLCK VI genes in Arabidopsis organs}

Figure 8A shows the relative transcript level of RLCK VI genes and a reference gene (GAPDH) normalized to the ACTIN 2/8 transcript level in Arabidopsis organs. Relative expression level is shown in relation to the average expression of each individual gene in all the investigated organs.

The relative gene expression levels were highly variable among the genes as well as the organs. However, it could be observed that the range of expression (absolute and relative as well, see Figure 7 and 8 A, respectively) is wider in the case of RLCK VI group A than for group B members (with exception of the RLCK VI_B3 and B4 gene pair). Only some small changes could be observed in the relative transcript abundance for the genes RLCK VI_B1, B2, B5 and B6. Most of the RLCK VI genes of Arabidopsis were only weakly expressed in the exponentially dividing cultured cells and transcripts of six of them could hardly be detected in this sample. Only the genes RLCK VI_A1, A4, A5, and B1 exhibited expression levels at or above the average in dividing cells, and for RLCK VI_A1 the relative expression was the highest in this sample. Similarly, only few RLCK VI genes showed increased expression in rosette and/or 
cauline leaves including RLCK VI_A2, A4, B4, and B7, the latter exhibiting the highest relative expression in this organ. The genes RLCK VI_A4, B3, and B4 were expressed at a level above average in the root. Several genes exhibited above average expression in the inflorescence stem and the gene RLCK VI_A5 showed the highest expression in this organ. The RLCK VI_A6 and A7 genes exhibited a very high relative expression in flower buds and open flowers and the RLCK VI_A3 gene had also its highest relative transcript level in the open flower. Figure 8B shows that this elevated expression detected in the flower indeed indicates strong expression in the pollen as can be deduced from microarray experiment data stored in the Genevestigator database (https://www.genevestigator.ethz.ch; Zimmermann et al. 2004, 2005).

In general, the data obtained by qRT-PCR in relation to the gene expression in the Arabidopsis organs were in good correlation with similar data available from the Genevestigator database as microarray datasets (see supplementary material in Jurca et al. 2008). However, in order to have the best match, the microarray data had to go through similar normalization steps used for the analysis of the qRT-PCR results.

In Figure 8, it can be recognized despite the high general variability in the overall gene expression patterns, that some of the paralogous gene pairs exhibit very similar (e.g. RLCK VI_A6 and A7) or rather complementary (e.g. RLCK VI_A1 and A2, B3 and B4) relative gene expression patterns in the various plant organs. 

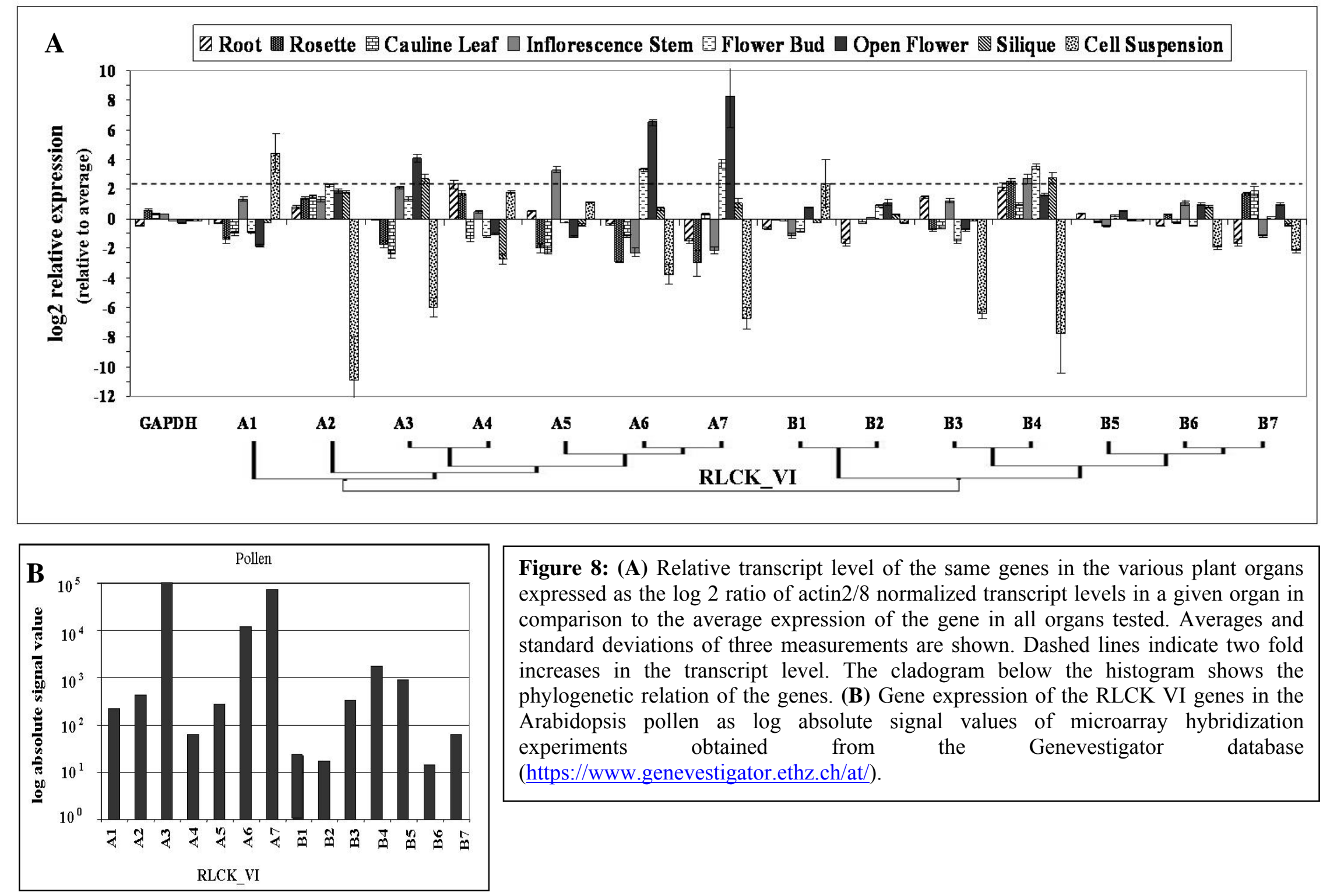

Figure 8: (A) Relative transcript level of the same genes in the various plant organs expressed as the $\log 2$ ratio of actin $2 / 8$ normalized transcript levels in a given organ in comparison to the average expression of the gene in all organs tested. Averages and standard deviations of three measurements are shown. Dashed lines indicate two fold increases in the transcript level. The cladogram below the histogram shows the phylogenetic relation of the genes. (B) Gene expression of the RLCK VI genes in the Arabidopsis pollen as $\log$ absolute signal values of microarray hybridization experiments obtained from the Genevestigator database (https://www.genevestigator.ethz.ch/at/). 


\subsubsection{Expression analysis of AtRLCK VI during various treatments}

In order to clarify the potential role of these kinases in stress responses, the differential gene expression of all RLCK VI members was tested in response to various abiotic stress and stress-related hormone (abscisic acid, salicylic acid, and ethylene) treatments for $6 \mathrm{~h}$. The normalized (ACTIN 2/8 as reference gene; geNorm method (Vandesompele et al. 2002) stressrelated expression of the genes was compared to gene expression in mock (normal culture medium) treated plantlets (Figure 9 A, B). As a positive control, the relative expression of the stress-responsive RD29A gene (Charrier et al. 2002; Yamaguchi-Shinozaki and Shinozaki 1993) was also determined in the same samples (Figure $9 \mathrm{C}$ ). The transcript level of the positive control RD29A gene was considerably increased in response to the treatments (from approximately tenfold in response to ethylene up to more than 1.000-fold in response to salt stress or ABA), verifying the effectiveness of the treatments and responsiveness of the seedlings.

As the 14 RLCK genes are considered, only few of them (RLCK VI_A1, A2 and A3, B2 and B3) showed significantly (more than fivefold) increased expression in response to stress/hormone treatments and only one of those carrying the UspA homology domain (RLCK VI_A1) was found amongst them. Only a moderate (at or slightly above twofold) increase could be observed in the case of the UspA domain-carrying RLCK VI_B4, B5 and B7 genes in response to some of the treatments. It could also be observed that if a gene proved to be stressinduced it was a rather general response, although the response to osmotic, sugar, and salt stresses, were the most prominent. The gene RLCK VI_B2 showed increased expression in all treatments except the cold and paraquat (oxidative stress), RLCK VI_B3 was unresponsive only to cold and salicylic acid while RLCK VI_A1 and RLCK VI_A3 only to cold. On the other hand, the gene RLCK VI_A2 exhibited approx. eightfold increased transcript level only in ABA treated samples and this gene proved to be the more cold responsive (approx. threefold increase). 

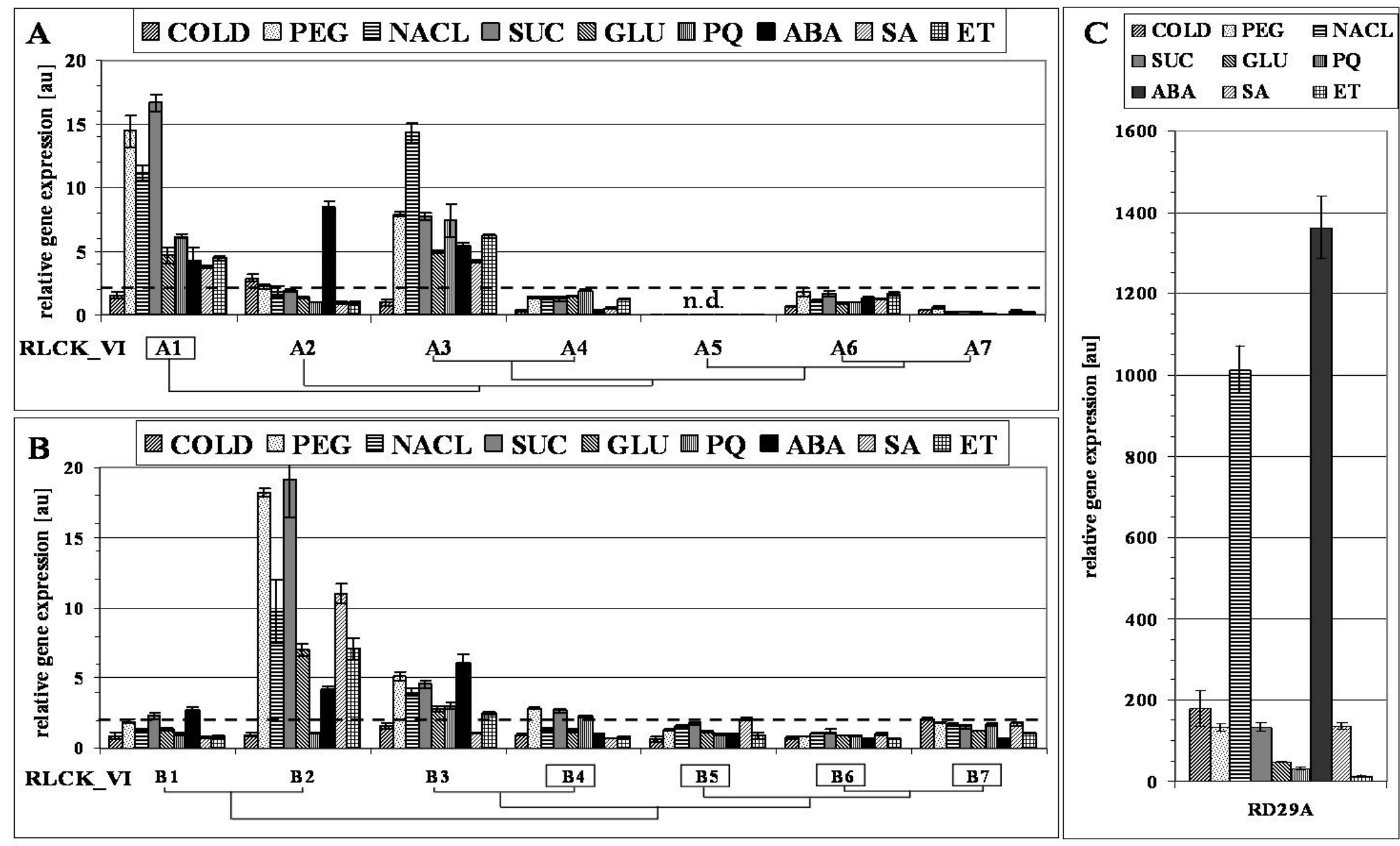

Figure 9: Relative expression of the Arabidopsis RLCK VI genes in response to stress, and stress-related hormone, treatments. (A) Relative expression of the RLCK VI group A; (B) Relative expression of the RLCK VI group B genes in response to the treatments. The UspA domain-containing genes are boxed. Dashed lines indicate twofold increase in the transcript level; (C) Relative transcript levels of the stress-inducible positive control RD29A gene in the same experiments. Cold $\left(4^{\circ} \mathrm{C}\right)$; PEG $(30 \%$ polyethylene glycol); $\mathrm{NaCl}$ (300 mM NaCl); Suc (8\% sucrose); Glu (300 mM glucose); PQ (25 $\mu \mathrm{M}$ paraquat); ABA (100 $\mu \mathrm{M}$ abscisic acid); SA (10 mM salicylic acid); Et (10 $\mathrm{mg} / \mathrm{l}$ etephon) 
ABA-induced expression could be observed for six RLCK VI genes (RLCK VI_A1, A2, and A3, B1, B2, and B3) and four of them also responded positively to salicylic acid and ethylene (RLCK VI_A1, A3, B2, and B3).

The genes not mentioned above either responded to the treatments with decreased gene expression (e.g. RLCK VI_A7) or exhibited only less than twofold increase in their transcript level. These genes include those having the highest relative transcript level in the reproductive organs, and therefore their stress/hormone responsiveness might not be the best investigated at the seedling stage, although they have significant relative expression in vegetative tissues, e.g., in the root, as well (Figure $8 \mathrm{~A}$ ). The transcript level of the RLCK VI_A5 gene did not reach the detection level in whole seedlings, even following the stress treatments, in accordance with its generally low expression level in all organs except inflorescence stem (Figure 8A).

No paralogous gene pair was found where both members exhibited strong stress response.

\subsubsection{Correlative expression between AtRLCK VI kinases and known/hypothesized ROP regulators and effectors}

Previous experiments indicated that AtRLCK VI kinases may serve as ROP GTPase effectors: a direct interaction of plant ROP GTPases with receptor-like cytoplasmic kinases (AtRLCK VI_A4 and A6; Molendijk et al. 2008) has been reported in Arabidopsis and could also be detected in alfalfa (Medicago truncatula RLCK VI_A4 and A6; Dorjgotov et al. 2009).

It is well known that plant ROP GTPases play central roles as signaling molecules during many basic cellular processes including cell shape determination, polar growth and responses to hormones, stress factors or pathogens. ROP GTPases have the potential to interact with a plethora of regulators and effectors that finally determines their signaling specificity. A comparison of gene expression patterns of the AtRLCKs, ROPs and their regulators and effectors may help to reveal potential signaling chains with biological relevance. In this direction, a hierarchical clustering analysis of microarray expression data was performed using the Genvestigator database and analysis tools (Figure 10). 


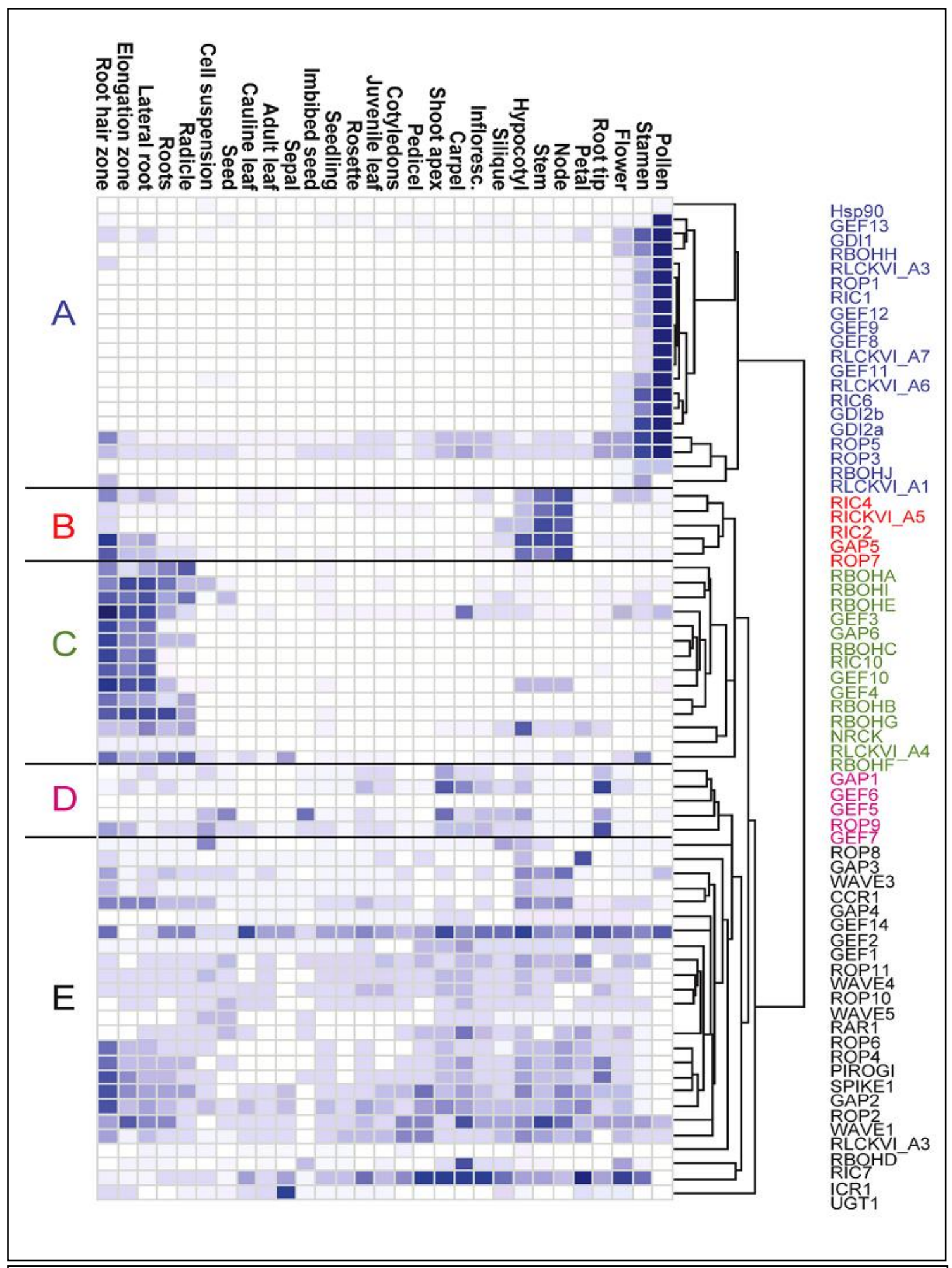

Figure 10: Expression pattern of ROPs and their known/hypothesized regulators and effectors including RLCK VI proteins. Relative gene expression is shown based on microarray data stored in the Genevestigator (Grennan 2006; Zimmermann et al. 2004) database and analyzed by a hierarchical clustering algorithm of the Genvestigator tool. Darker blue color means higher relative expression. Main clusters (A-E) are separated by lines and the corresponding genes are highlighted by the same color. 
Figure 10 shows that many of the investigated genes are expressed in several organs/tissues, making it difficult to predict functional interactions among them. However, several proteins of these families show a preferential expression in only a small subset of organs/tissues and the correlation of the relative expression pattern of several putative partners is evident. Five main expression clusters (A-E) could be established. These clusters include genes with preferential or more abundant expression in the pollen and flower (clusterA), stem, node and hypocotyl (clusterB), root (clusterC), shoot and root tip, flower and silique (clusterD), and all over the plant (clusterE). Cluster E contains the highest number of genes with relatively ubiquitous expression, although preferential expression in some organs/tissues can be observed for individual genes even within this family.

The cluster A form a group of genes with high and specific expression in pollen. These genes include AtRLCK VI_A3, A6 and A7; genes for ROP1, 3 and 5 (with ROP3 and 5 expressed in many other tissues as well); guanine nucleotide exchange factors, regulators of GPTase activity (GEF8, 9, 11, 12 and 13); guanine nucleotide dissociation inhibitors (GDI1, 2A and 2B), with GDI1 also expressed at a relatively high level in vegetative tissues, especially in the root; RIC1 and 6; and two NADPH complex subunits $\mathrm{RBOHH}$ and $\mathrm{J}$.

The root-enhanced expression of several other members is also clear and well defined (cluster C). Interestingly, many NADPH complex subunits (RBOHA, B, C, E, F, G, I) belong to this cluster. Although ROP2 (Jones et al. 2002), ROP4 and 6 (Molendijk et al. 2001) GTPases have been shown to play role in root hair growth, they exhibit a more ubiquitous expression pattern (Figure 10). Their root specific interacting partners are not known, but PIROGI, SPIKE1, GAP2 and WAVE1 have the most similar expression pattern while RIC10, GAP6, GEF3, 4 and 10 might be suggested as further candidates based on their preferential expression in the root hair zone (Figure 10). The AtRLCK VI_A4 is also included in this cluster, with only a low expression level.

The AtRLCK VI_A5, are also expressed in the root hairs but it form a specific group together with RIC4, ROP7, GAP5, RIC2 due to their high relative expression in stems, nodes and hypocotyls as well (Figure 10). The qRT-PCR data showed that AtRLCK VI_A5 had a higher expression level in inflorescence stems (Figure 8A). 
WAVE3, CCR1 and GAP4 are also expressed at a relatively high level in these organs although belong to group $\mathrm{E}$ with a more ubiquitous expression pattern. ROP9, GEF5, 6, 7 and GAP1 exhibit relatively high expression in root and shoot tips in addition to other tissues. Therefore they may be important for meristem formation and maintenance. ICR1 has also been shown to affect root meristem formation (Lavy et al. 2007), although it shows stronger accumulation in the flower (petal, sepal) and the shoot apex (Figure 10).

\subsection{Specific interaction of AtRLCKs VI_A kinases with AtROPs in yeast two-hybrid assay}

Concerning the potential role of AtRLCKs in signal transduction pathways, only limited information is available. In our laboratory, by carrying out a yeast two-hybrid screening using the MsROP6 GTPase $\mathrm{CA}+\mathrm{L}$ (constitutively active and non-prenylated mutants), two RLCKs, named MtRRK1/2 (Medicago truncatula ROP-interacting Receptor-like Kinases), were identified (Dorjgotov et al. 2009). BLAST searches with the sequence of the two alfalfa-RRKs have shown that they are highly homologous to kinases in the RLCKs Class VI family of Arabidopsis. Therefore, we decided to test whether the Arabidopsis RLCK VI kinases are also capable to interact with ROPs. Moreover, we addressed the specificity of interaction using all the available Arabidopsis and Medicago ROP and RLCK VI kinase cDNA clones.

For this purpose, cDNA clones of 8 different Arabidopsis thaliana ROP GTPases, 10 Arabidopsis RLCK VI kinases (5 from group A, 5 from group B) and three further kinases belonging to other RLCK families (IV, VII, IX) were inserted into yeast twohybrid vectors. The MtRRK1 and MtRRK2 (orthologs of Arabidopsis RLCK VI_A2 and A5) kinases and the MsROP6 GTPase were also included into the experiment as a control.

The full length cDNAs of the Arabidopsis and Medicago kinases were cloned into the activation-domain vector, pAD-GAL4 2.1 and full length cDNAs of Arabidopsis ROPs were cloned into the DNA-binding domain vector, pBD-GAL4 2.1. The nonprenylated (localization, L, mutation) variants of AtROPs were used to allow the AtROP fusion proteins to accumulate in the yeast nucleus as a requirement for successful assays (see Material and methods). Because, the Rho GTPases interact with effectors, preferentially in their GTP-bound state the constitutively active form $(\mathrm{G} 15 \mathrm{~V})$ of 5 
different Arabidopsis ROP GTPases (AtROP1, 4, 6, 8 and 9) were also included into the experiment.

Moreover, to exclude the interaction of the protein of interest with e.g. the DNA activating or binding region, yeast strain was co-transformed with empty pAD-GAL4 2.1 and pBD-AtROPs (WT and CA variants), respectively empty pBD-GAL4 2.1 and pADGAL4 2.1-RLCKs. Protein-protein interactions were detected in double (-Trp,-Leu), triple (-Trp,-Leu,-His) and four (-Trp,-Leu,His,-Ade) selection plates. The data from the double selection (-Trp, -Leu), which indicate the presence of both constructs and the four (-Trp,-Leu,His,-Ade) selection are not shown. The data from the triple (-Trp,-Leu,-His) selection, which indicated the positive interactions are presented in the Figure 11. The experiment was repeated several times (2-to-4 times depending on the partners) with the same results.

The yeast two-hybrid interaction matrix (Figure 11) indicated that all but one investigated AtRLCK VI_A kinases but none of the studied AtRLCK VI_B or AtRLCK IV, VII, or IX kinases showed interaction with the Arabidopsis or Medicago ROP GTPases involved into the experiment although the expression of all RLCKs in the yeast cells was confirmed by a western blot (data not shown). The MtRRK1 kinase and its Arabidopsis homologue, AtRLCK VI_A2, could interact with all investigated ROP GTPases independent of their WT or CA conformation. The MtRRK2 kinase did not show interaction only with the AtROP10 GTPase (the cDNA clone of the Arabidopsis homologue of the MtRRK2-kinase could not be isolated and tested). Interactions of the other AtRLCK VI_A kinases could readily be detected only with the CA versions of the investigated GTPases.

These data indicate that different groups of ROP GTPases have a general potential to interact with AtRLCK VI_A members, and mark these previously uncharacterized protein kinases for participation in ROP-regulated processes.

In order to identify structural elements responsible for the specificity of ROP interaction with RLCK VI_A kinases we looked for differences in the primary structures of the RLCK VI_A and B kinases that might lead us to the identification of distinct regions/motives, which might play a role in the specificity of interaction. 


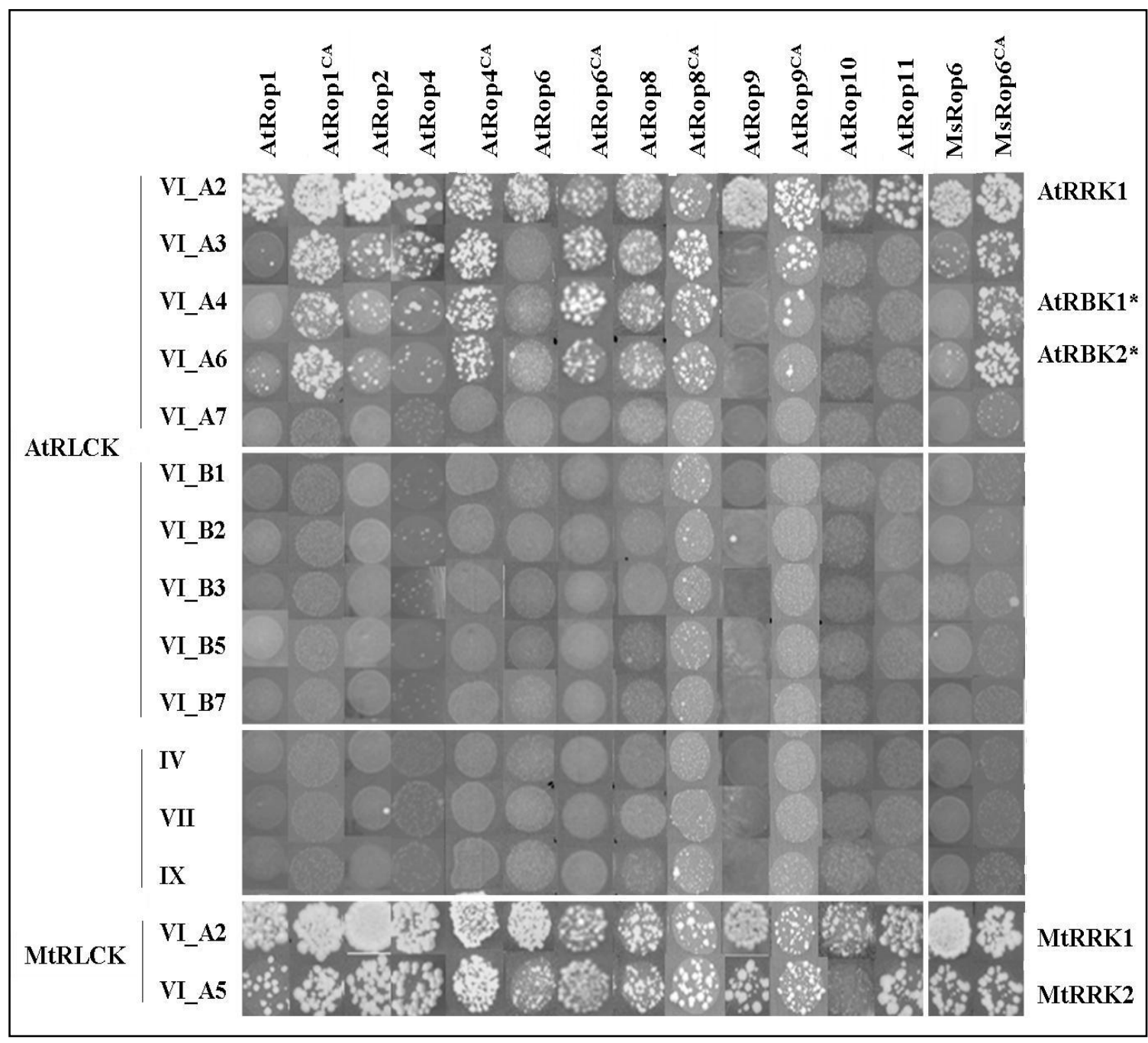

Figure 11: Yeast two-hybrid interaction matrix of selected Arabidopsis and Medicago RLCK kinases and ROP GTPases. RLCK kinase and ROP GTPase genes were pair wise expressed in yeast cells as indicated. The growth of transformed yeast cells on Trp-, Leu-, His- drop out medium in the presence of $1 \mathrm{mM} \mathrm{3-aminotriazol} \mathrm{is} \mathrm{shown.} \mathrm{Wild} \mathrm{type} \mathrm{and} \mathrm{in} \mathrm{some} \mathrm{cases}$ constitutive active (CA) ROP GTPases were tested for the interaction. *AtROP4-binding RLCK kinases described by Molendijk et al. 2008.

\subsection{Protein sequence analysis reveals differences between $A$ and B class of AtRLCKs VI family}

None of the ROP-interacting RLCK kinases have any recognizable sequence motif known to play role in the binding of Rho-type GTPases to their effectors in yeast and animals.

In an attempt to elucidate the residues or motifs that might be essential for the interaction of RLCKs and ROPs, the primary sequences of Arabidopsis kinases known to 
interact or not to interact with ROPs were compared. The sequence alignment included all 14 members of the At RLCK VI family consisting of the ROP-interacting RLCK VI_A and the non-interacting RLCK VI_B group. Three further kinases that belong to RLCK Class VII and VIII (NAK, Ptil) or to mitogen-activated protein kinases (AtMPK1) were also included into the analysis.

Multiple alignments of these sequences are shown in Figure 12. Because, the length of $\mathrm{N}$-and $\mathrm{C}$-terminal regions proved to be highly variable only the region exhibiting high homology is presented. The result shows that there are characteristic sequence differences between kinases belonging to the RLCK VI_A and B groups differing in their ROP-binding capability (Figure 12). Therefore, this analysis allowed us to identify candidate residues/motives that may be responsible for RLCK-to-ROP GTPase binding. The importance of these regions in the molecular interaction will be experimentally verified in the future.

Furthermore, we could not identify any region that is characteristic for the RLCK VI group A members that is not conserved in AtRLCK VI_A7. From the primary sequence comparison, we could not find out the reason why this specific kinase from RLCK VI group A worked differently in our assay. 


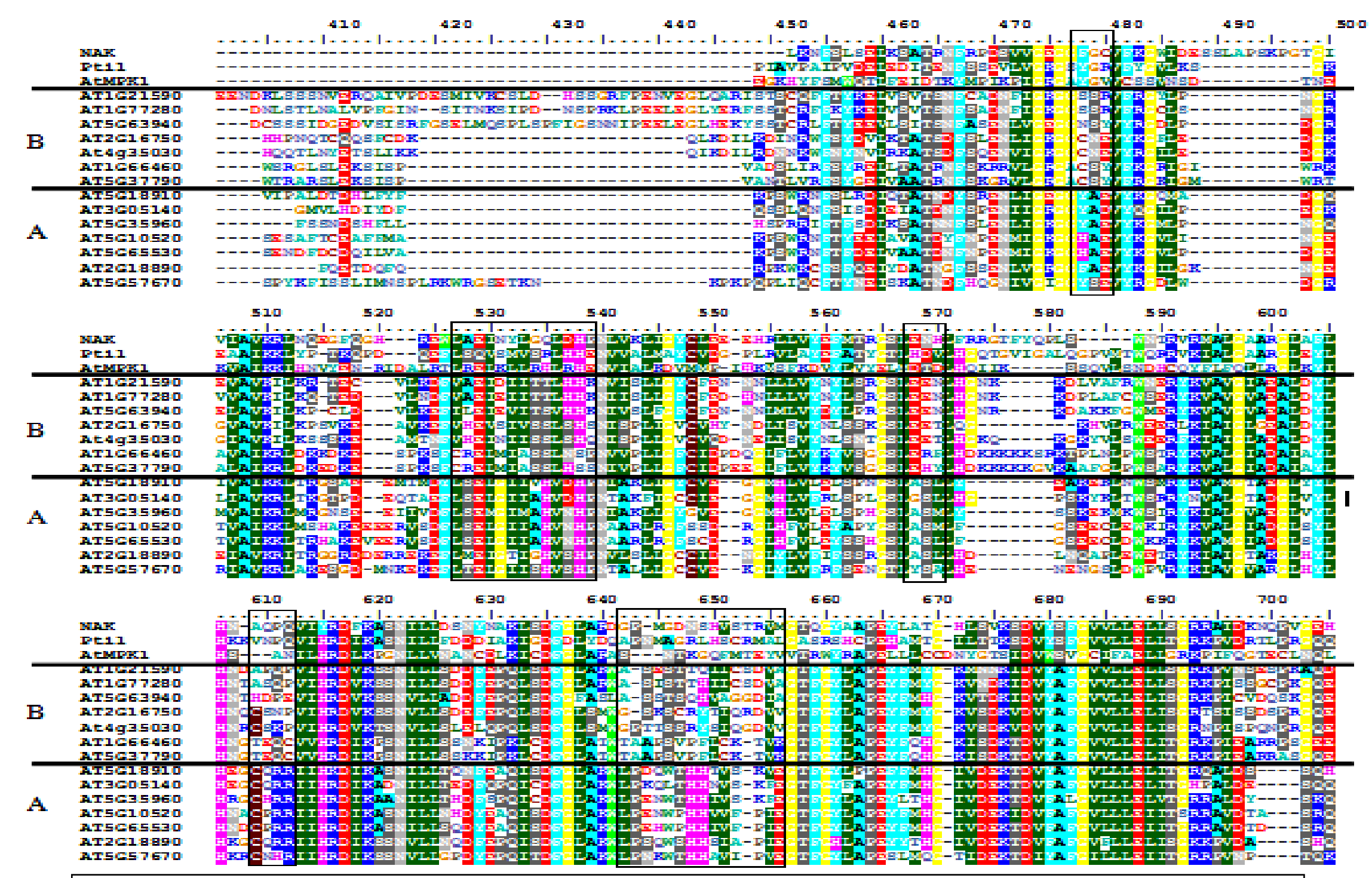

Figure 12: Comparison the primary sequence of the AtRLCK VI proteins (A - group A; B -group B) and three other protein kinases from different classes: NAK and Pti1 are RLCK from class VII and VIII, respectively, and AtMPK1 is a mitogen-activated protein kinase. The three kinase groups (group A, group B and the others) are separated by lines. The motives conserved only in the sequences of the AtRLCK VI_A group are boxed. 


\subsection{Arabidopsis RLCKs VI kinases interact with AtROPs in vitro}

In an attempt to confirm the yeast two-hybrid interaction identified between AtRLCKs VI group A and AtROPs, an in vitro binding assay was performed. In this procedure, AtROP1 (WT and CA form) tagged with MBP (maltose binding protein) and coupled to amylose resin was used as an affinity matrix for the purification of AtRLCK VI-A2 or AtRLCK VI_B3, respectively. In yeast two-hybrid assay the AtRLCK VI_A2 have been shown to interact with all the AtROPs tested while, in contrast, AtRLCK VI_B3 did not interact with any AtROPs.

The full coding sequences of wild-type (WT) and CA (constitutively active) AtROP1 (594 bps) were fused at their N-termini to maltose binding protein (MBP) using the pMAL2c and pMAL_DEST expression vectors, respectively. The full coding sequences of the AtRLCKs VI_A2 (1179 bps) and B3 (966 bps) were tagged at their Ntermini with hexahistidine (6xHis) sequences using the pET28c vector.

For the interaction assay, the amylose resin bounded AtROP1_CA and AtROP1 (WT) in nucleotide depleted, GTP $\gamma \mathrm{S}$ - and GDP-bounded states were incubated for $2 \mathrm{~h}$ with the purified AtRLCK VI_A2 or B3 kinases. As control, the MBP protein and the amylose resin were also incubated with purified AtRLCK_A2 or B3 proteins. Western blot analysis and immuno-detection were used to visualize protein-protein interactions, using a Peroxidase-conjugated anti-poly histidine, anti-maltose-binding and anti-rabbit IgG antibodies (MBP:AtROP1 at about 67kDa and the HIS:AtRLCK VI_A2 and B3 at about $49 \mathrm{kDa}$ and $42 \mathrm{kDa}$ ). By performing this assay, it was addressed whether AtRLCKs bound to specific states of the ROP GTPase in vitro. The interactions of AtRLCK VI_A2 and B3 with different forms of AtROP1 are shown in Figure $13 \mathrm{~A}$ and B.

As shown in Figure 13 A, AtRLCK VI_A2 was pulled down by AtROP1 in all three forms, nucleotide depleted, GTP $\gamma$ S- and GDP-bounded, but not by control MBP (maltose binding protein). This indicated that AtRLCK VI_A2 binding to AtROP1 protein was independent of the activation state of the GTPase.

When the assay was done with AtRLCK VI_B3 (Figure 13 B), the results showed that this kinase could also form complexes with AtROP1, which is opposite to the yeast two hybrid assay results (Figure 11). AtRLCK VI_B3 could bind to all conformations of AtROP1 with more-or-less the same affinity. In the control samples, no bands were 
detected, showing that AtRLCK VI_B3 did not interact with MBP or amylose beads. Repetition of these experiments showed that the results were reproducible.

These data indicate that there are significant differences in the sensitivity of the various approaches used to detect protein-protein interactions (for review see Lalonde et al. 2008). This might be explained among others (see Disccussion and Conclusion) by the various conformations and levels of the proteins available for interaction in solution or in yeast nuclei.

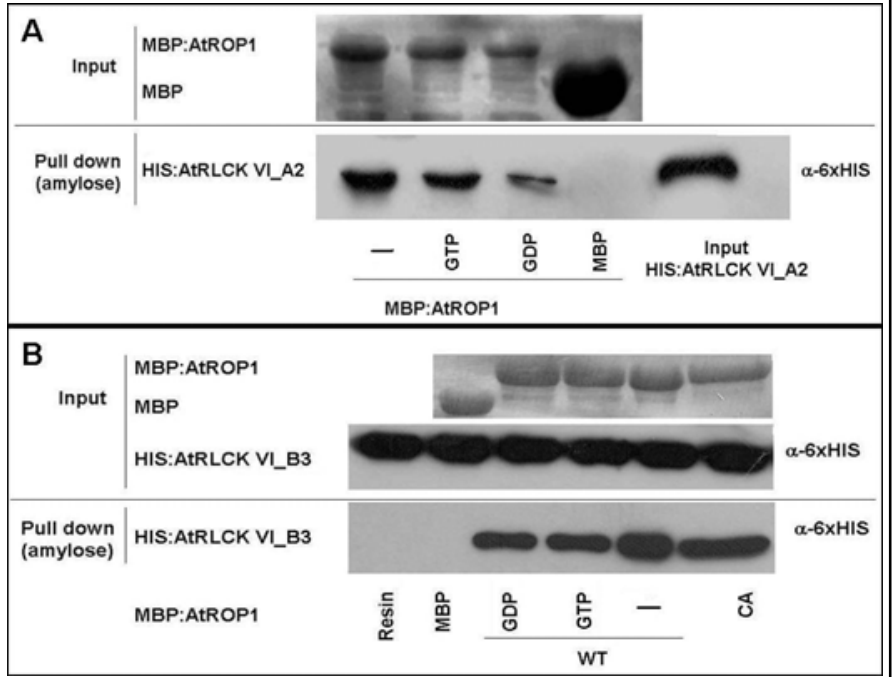

Figure 13: In vitro pull down assays using purified 6xHIS-tagged RLCK VI kinases, (A) HIS:AtRLCK VI_A2 and (B) HIS:AtRLCK VI_B3, as preys and maltose-binding protein-tagged AtROP1 GTPase (MBP:AtROP1) as bait in various forms (CA, and WT in the presence or absence (-) of GTP or GDP, respectively). Maltose binding protein (MBP) and pure amylose resin (Resin) were used as controls. Proteins detected on the Western blots are indicated on the left, the used antibodies on the right. The relative amounts of the input proteins are shown also.

\subsection{AtRLCK VI_A kinases are specifically activated by active ROP GTPases in vitro}

In order to test whether the kinase activity of Arabidopsis RLCKs is dependent on ROP GTPases, in vitro kinase assays were performed. In this procedure, two AtRLCK VI which belongs to the group A (RLCK VI_A2 and A6), one AtRLCK VI from group B (RLCK VI_B3) and one RLCK that belong to a different class (RLCK VII), were tested with purified AtROP1 and MsROP6. These proteins were over expressed in E. coli and purified as described in the Materials and Methods chapter.

For kinase activity measurement, each reaction was run with 2 pmol purified kinase, 5 pmol purified small GTP binding protein and $0,25 \mu \mathrm{g} / \mu \mathrm{l}$ myelin basic protein (a universal substrate for serine/threonine kinases). After the reaction was performed for 30 minutes at room temperature, the protein samples were separated by SDS polyacrylamide gel and the dried gels were analysed by a Phosphorimager 445 SI station. 
In vitro activity of the AtRLCK VI_A2 kinase in the presence and absence of ROP GTPase is shown on Figure 14. AtRLCK VI_A2 was named AtRRK1 as well, because it is the ortholog of Medicago MtRRK1. In this experiment two purified ROP GTPases were included, one form Arabidopsis (AtROP1) and one from Medicago (MsROP6) which allowed us to check the interaction and activation specificity. The data showed that in the absence of GTPase the phosphorylation of myelin basic protein (MyBP) was hardly detectable. The in vitro MyBP phosphorylating activity of the AtRLCK VI_A2 kinase (AtRRK1) was increased by Medicago (MsROP6) as well as Arabidopsis (AtROP1) GTPases in the presence of GTP. A lower level of phosphorylation of the substrate was observed in the presence of ROP GTPases without the addition of GTP/GDP or when GDP is added to the reaction.

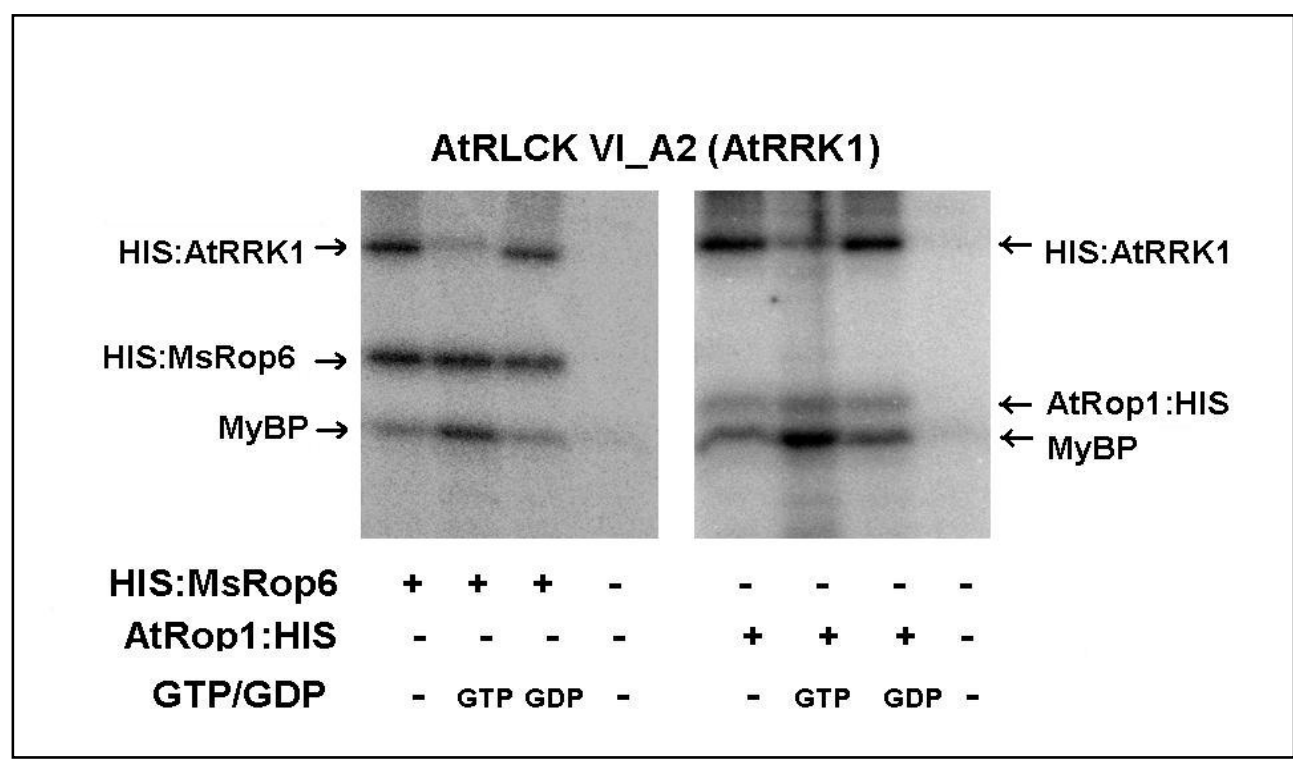

Figure 14: In vitro activity measurement of the AtRLCK VI_A2 kinase in the presence and absence of ROP GTPases (AtROP1 and MsROP6, respectively) in the absence or presence of GDP/GTP.

Autophosphorylation of the kinase was also detected and it was dependent on the presence of the GTPase in the active conformation. The autophosphorylation take place at the $\mathrm{N}$-terminus and is not required for in vitro activity (Dorjgotov et al. 2009). The reason why autophosphorylation is decreasing in the presence of GTP is not known, but the same could be observed for Medicago RRK1 and 2 kinases (Dorjgotov et al. 2009). 
The N-terminally HIS-tagged MsROP6 GTPase could also be efficiently phosphorylated by the kinase in contrast to the C-terminally HIS-tagged AtROP1. We found previously that this is due to addition of a phosphorylatable serine residue through the cloning procedure to the $\mathrm{N}$-terminus (but not the $\mathrm{C}$-terminus) of the GTPases (Dorjgotov et al. 2009). RLCKs do not phosphorylate ROPs as could be proved when a C-terminally HIS-tagged ROP GTPase was used in the kinase assay (Dorjgotov et al. 2009).

The data showed that the AtRLCK VI_A2 kinase could be activated in vitro by both AtROP1 and MsROP6 GTPases and that the presence of the GTPases in GTP-bound conformation was the most efficient in increasing the phosphorylation activity of the kinase. These data were in agreement with those obtained by Medicago RRK kinases and ROP GTPases (Dorjgotov et al. 2009)

In order to test the specificity of ROP GTPase-mediated activation of various RLCK kinases, the activities of the Arabidopsis kinases AtRLCK VI_B3, representing the B group of RLCK VI, and a further kinase belonging to RLCK Class VII were also tested. The kinase activity was determined in the presence of C-terminally HIS-tagged MsROP6 GTPase (Figure 15). The GTPase could not activate in vitro the AtRLCK VI_B3 or the AtRLCK VII-type kinases in contrast to the RLCK VI_A2 kinase (Figure 14).

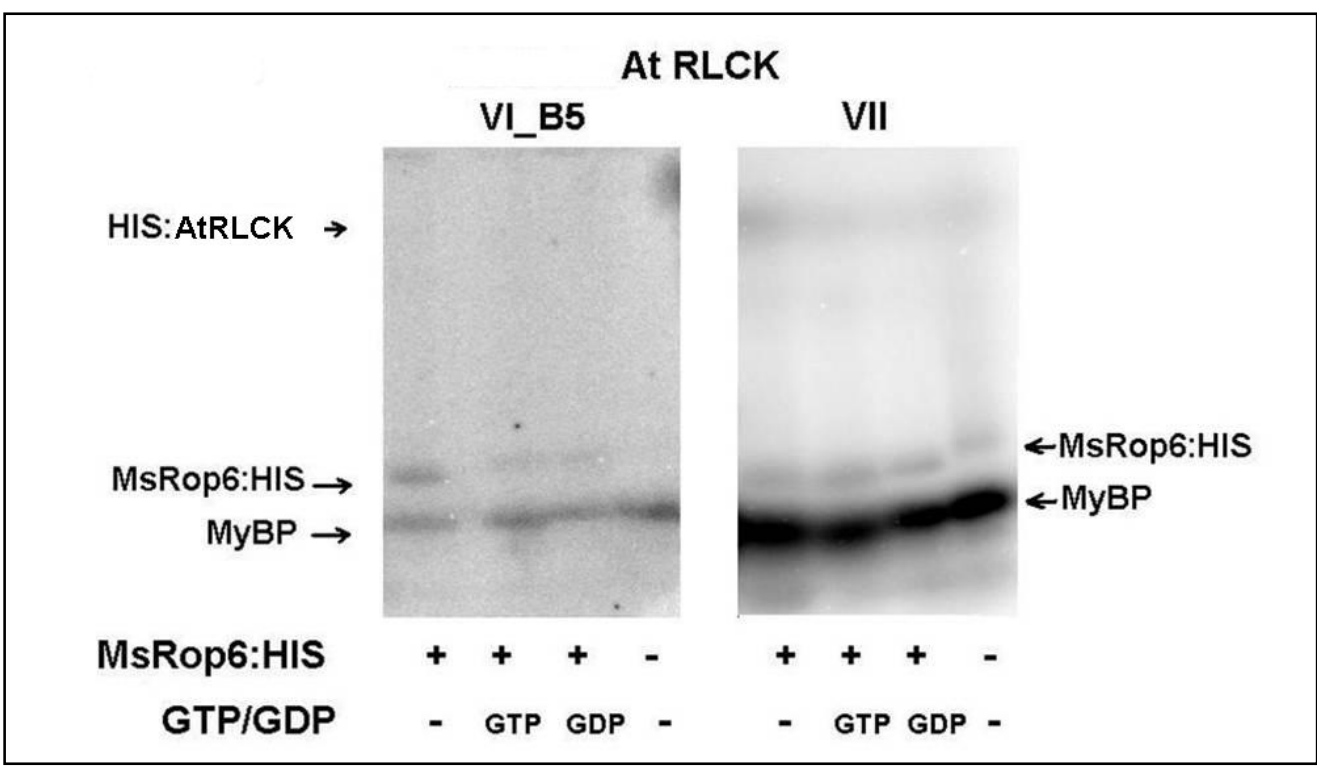

Figure 15: In vitro MyBP phosphorylating activity of representing members of the RLCK VI group B (VI_B5), and RLCK VII (VII) families in the presence or absence of the Cterminally HIS-tagged MsROP6 GTPase with or without GTP/GDP, as indicated. The position of the autophosphorylated HIS:AtRRK1 kinase is also indicated. 
As it was mentioned previously, during the course of our investigations, a German research team reported that two AtRLCKs from class VI, AtRBK1/AtRLCK VI_A4 and AtRBK2/AtRLCK VI_A6 and a cysteine-rich receptor kinase (NCRK, RLCK VIII) are able to interact in vitro and in vivo with the AtROP4 GTPase (Molendijk et al. 2009). However, they could not demonstrate that those kinases are activated by the ROP GTPase.

Therefore the activity of one of these RLCK VI kinases (AtRLCK VI_A6/RBK2) in the presence of ROP GTPase was also tested (Figure 16). The AtRLCK VI_A6/RBK2 was able to significantly phosphorylate the myelin basic protein (MyBP) only if the HIS:AtROP1 CA mutant was present. In the presence of WT HIS:AtROP1, MyBP phosphorylation was barely observed. These data further support the ROP GTPase dependent activity of Arabidopsis RLCK VI_A kinases.

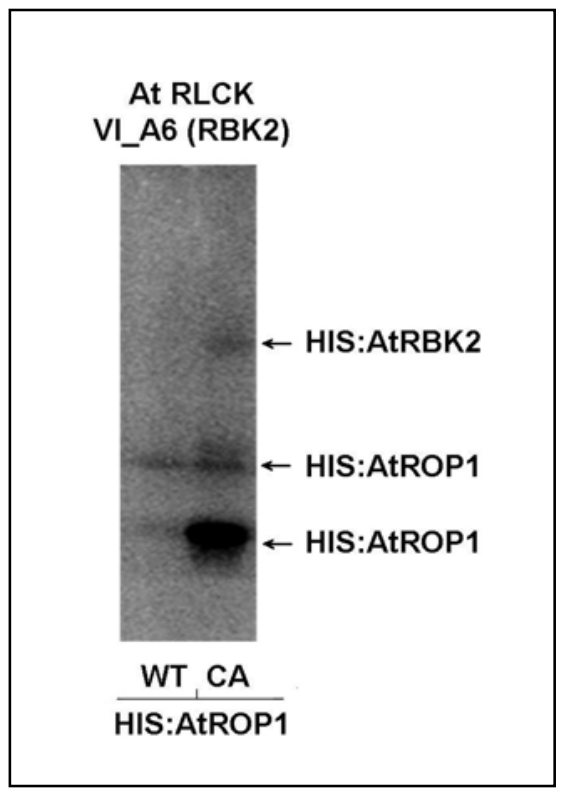

Figure 16: In vitro MyBP phosphorylating activity of AtRLCK VI_A6/RBK2 in the presence of N-terminally HIS-tagged AtROP1 GTPase, WT and CA (constitutively active) forms. The position of the autophosphorylated HIS:AtRLCK VI_A6/AtRBK2 kinase is also indicated.

It can be concluded that ROP GTPases are capable for the specific activation of AtRLCK Class VI group A kinases. However, it is not clear how the GTPases can activate the kinases.

In animals, it is well known that the most common mechanism of effector activation by Rho GTPases is the disruption of intramolecular autoinhibitory interactions, to expose functional domains within the effector protein. For example, RAC or Cdc42 that target PAK kinases bind to an N-terminal PAK domain that inhibit kinase activity and upon binding, the inhibitory sequence is displaced, leaving the kinase domain free to 
bind to substrates (Bagrodia and Cerione 1999; Tu and Wigler 1999). Once PAK kinase is activated through interaction with $\mathrm{Rac} / \mathrm{Cdc} 42$ it then autophosphorylates, which both decreases its affinity for GTPases and maintain also Pak kinases in an open, activated conformation even when dissociated from GTPases (Manser et al. 1994).

The ROP-activated RLCK VI_A kinase has neither a GTPase-binding CRIB motif nor an autoinhibitory domain and how the ROP GTPases are capable of activating these kinases is still unknown. Structural comparison of RLCK VI group A and B kinases may serve as a basis to identify important regions of the kinases for this functional interaction (see earlier and Figure 12)

In animal cells it is well know that $\mathrm{RHO} / \mathrm{RAC} / \mathrm{CDC} 42$ GTPases have a characteristic region, the RHO insert region, which has an important role in activation the effector proteins (Karnoub et al. 2001; Thapar et al. 2002). Moreover, it was demonstrated that variations of this region do not affect the interaction between the proteins (Freeman et al. 1996; Karnoub et al. 2001; Zong et al. 2001). In our earlier studies on Medicago ROPs, the Rho insert region was found to be important in the activation of MtRRK proteins but the deletion of this region did not affect the ability of the GTPase to interact with the effector kinase (Dorjgotov et al. 2009).

\subsection{Characterization of transgenic lines}

To analyze the roles of AtRLCK VI_A2 in plants, the gene was overexpressed or silenced in Arabidopsis plants. For overexpression we used two different binary vectors, heat-inducible and estradiol-inducible promoter vectors (pJCGLox and pER8) and for silencing an estradiol-inducible promoter vector (pER8RNAi).

\subsubsection{Selection of transgenic lines over-expressing AtRLCK VI_A2}

First step in analyzing the transgenic plants was to select for the lines which show altered level AtRLCK VI_A2 expression: from low to high overexpression and from decreased to eliminated expression in the overproducing and RNAi lines, respectively.

The expression level of the transgene was determined in $\mathrm{T}_{0}$ plants (heterozygous plants). The positive transformants which were selected on hygromycin containing medium for plants transformed with pER8-AtRLCK VI_A2 or on kanamycin containing medium for plants transformed with pJCGLox-AtRLCK_A2 were used following transfer 
into the greenhouse for seed setting. The transgene level was determined by using the techniques of semiquantitative PCR or qRT-PCR.

First, the transcript level of the transgene was checked in plants transformed with pER8-AtRLCK VI_A2. Leaves from several independent transgenic lines and nontransgenic plants were sprayed with $10 \mu \mathrm{M}{ }^{17} \beta$-estradiol and the transcript levels were determined at $6 \mathrm{~h}$ after treatment by using semiquatitative PCR. As control, non-treated samples were also included. 18S rRNA abundance was used as a reference control.

Different transgene expression levels could be detected for six out of the nine lines analyzed (Figure 17). Lines EOX-4, 6 and 8 showed a moderate increase in the transgene level. High level overexpression could be detected for the lines EOX-3, 7 and 11. Three lines (EOX-2, 21 and 24) showed no increase in the transgene level.

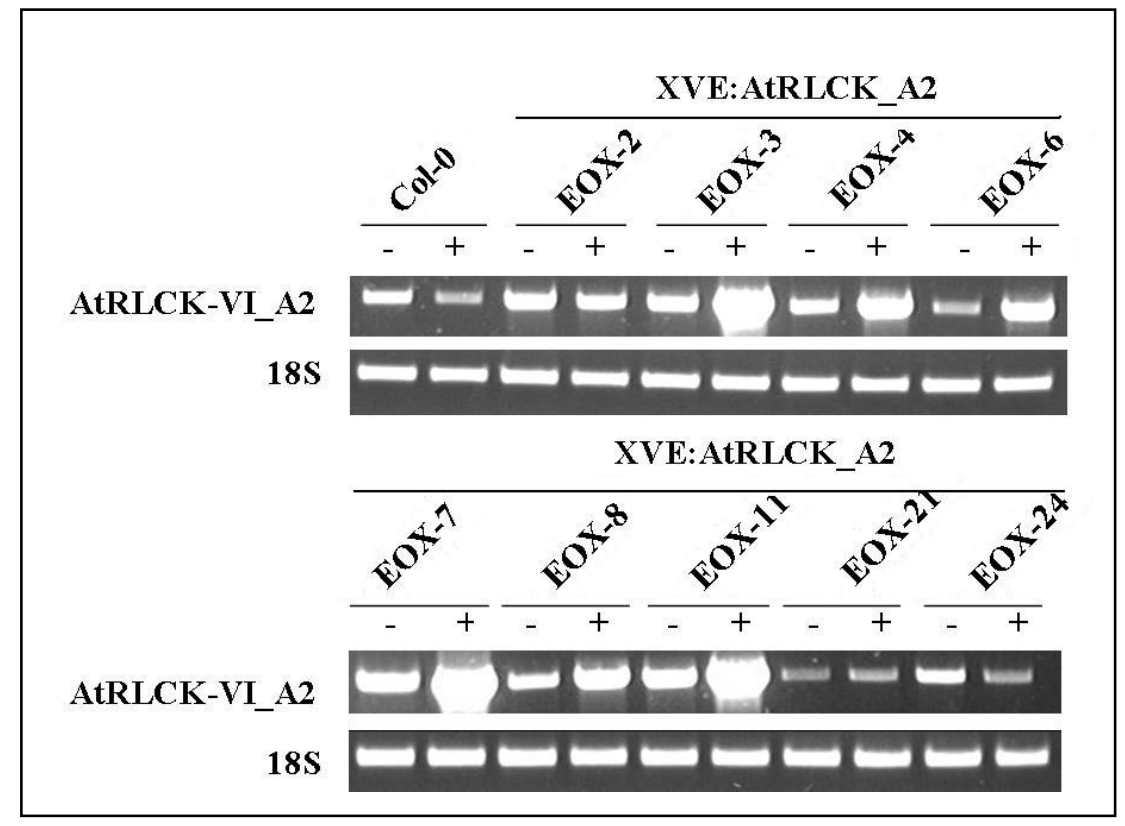

Figure 17: Semiquantitative RT-PCR analysis of pER8-AtRLCK VI_A2 transgenic lines. The transcript level in $\mathrm{T}_{0}$ plants was determined at $6 \mathrm{~h}$ after spraying the leaves with $10 \mu \mathrm{M}{ }^{17} \beta$ estradiol solution. Nine independent transgenic lines generated in the Columbia $(\mathrm{Col})$ ecotypic background were analysed; 18S rRNA was used as a control in each PCR reaction; (-) untreated control; (+) treatment with $10 \mu \mathrm{M}{ }^{17} \beta$-estradiol; EOX-2 to 24: estradiol inducible over-expressing lines

For checking the inducibility of transgene expression in plants transformed with the pJCGLox-AtRLCK VI_A2 construct, leaves from $\mathrm{T}_{0}$ transformants were incubated for $2 \mathrm{~h}$ at $37^{\circ} \mathrm{C}$. A non-treated control was included for every line analyzed. The relative 
expression level of AtRLCK VI_A2 transgene was determined at 24h after heat treatment, in twenty-one independent transgenic lines by using the qRT-PCR technique.

Varying expression level could be observed among the lines analyzed (Figure 18). Nine lines showed no increase in the level of the transgene upon heat induction (HOX-2, $5,9,10,16,17,19,20$ and 22). Moderate increase in the expression level was detected for seven lines (lines 1, 3, 6, 8, 11, 12 and 15). Lines HOX-4, 7, 14, 23 and 13 showed a high expression of the AtRLCK VI_A2 transgene after heat induction (Figure 18), with the highest expression level that was more then 70 -fold increased in comparison to the control in the line HOX-13.

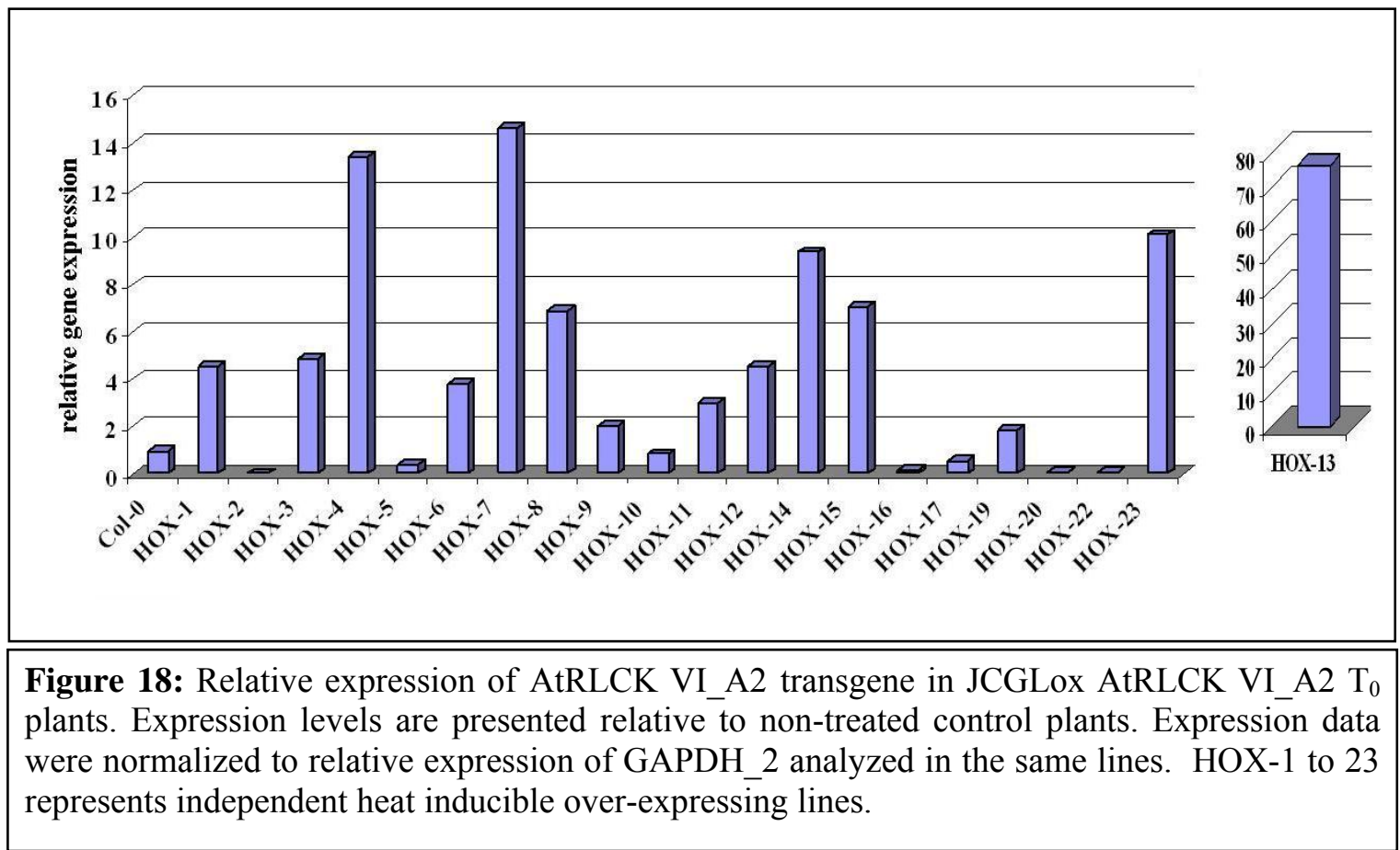

\subsubsection{Selection of transgenic lines silencing AtRLCK VI_A2}

The AtRLCK VI_A2 silencing in Arabidopsis plants transformed with pER8RNAi-AtRLCK VI_A2 construct was determined in several independent $\mathrm{T}_{0}$ transgenic lines. Leaves collected from transgenic and non-transgenic Arabidopsis plants were sprayed with $10 \mu \mathrm{M}{ }^{17} \beta$-estradiol and the decrease in transgene level was checked at $6 \mathrm{~h}$ after induction.

Transformants with reduced AtRLCK VI_A2 transcript level were identified by semiquantitative RT-PCR using AtRLCK VI_A2 gene specific primers that amplify the 
full length coding sequence. A control RT-PCR reaction was performed for every transgenic line using the primers that specifically amplify the $18 \mathrm{~S}$ rRNA.

Out of the eight independent transgenic lines analyzed, lines ERNAi-2, 5, 7 and 10 showed the greatest reduction in AtRLCK VI_A2 transcript level compared with the non-treated controls. A slight decrease in the transcript level could be detected for line 6 . Three independent lines (ERNAi-3, 4 and 8) did not show any decrease in the transcript level upon estradiol induction (Figure 19).

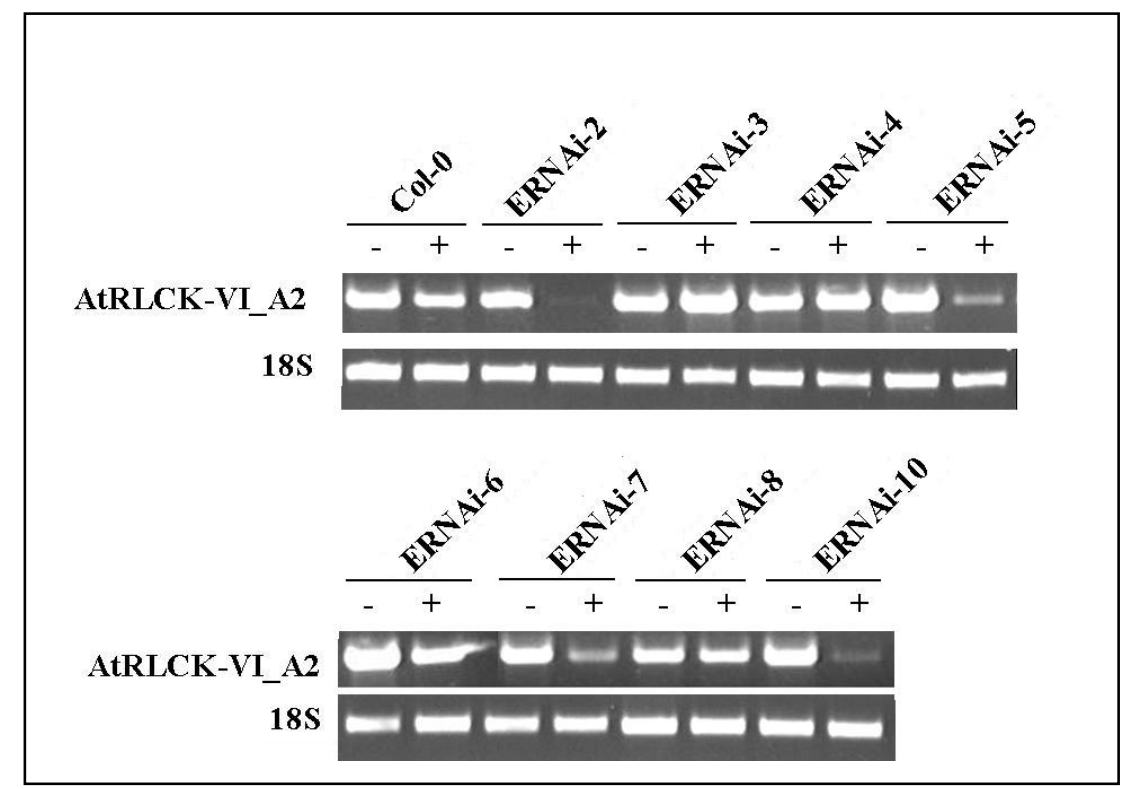

Figure 19: Semiquantitative RT-PCR analysis of pER8-RNAi-AtRLCK VI_A2 transgenic lines. The transcript level in $\mathrm{T}_{0}$ RNAi lines was determine at $6 \mathrm{~h}$ after spraying leaves with $10 \mu \mathrm{M} \beta$-estradiol solution. Eight independent transgenic lines generated in the Columbia (Col) ecotypic background were analyzed. 18S rRNA was used as a control in each PCR reaction; (-) untreated control; (+) treatment with $10 \mu \mathrm{M}{ }^{17} \beta$-estradiol; ERNAi 2 to 10: estradiol inducible RNAi lines.

\subsubsection{Trichome morphogenesis in over-expressing and silencing AtRLCK VI_A2 transgenic plants}

Trichome morphogenesis is a well known process regulated by ROP GTPases in plants (Ishida et al. 2008). To determine whether trichome development is also affected by the ROP-activated RLCK kinases, Arabidopsis lines overexpressing or silencing AtRLCK VI-A2 were analyzed. Trichomes were isolated from mature leaves of induced and non-induced transgenic lines ( $T_{1}$ generation) as well as from non-transformed plants at 3 weeks after germination. For each line, a number of 200-300 isolated trichomes were analyzed. 
A preliminary analysis of induced transgenic lines didn't show a sever modification in trichome morphogenesis. Over-expressing of AtRLCK VI_A2 induce a defect only in trichome branching. No any modification in trichomes distribution on the leaves or branch elongation could be observed in these mutants. Normally, leaf trichomes have three branches and only a small percentage of trichomes possess two or four branches as it could also be observed in our experiment (Figure $20 \mathrm{~A}, \mathrm{C}$ ).

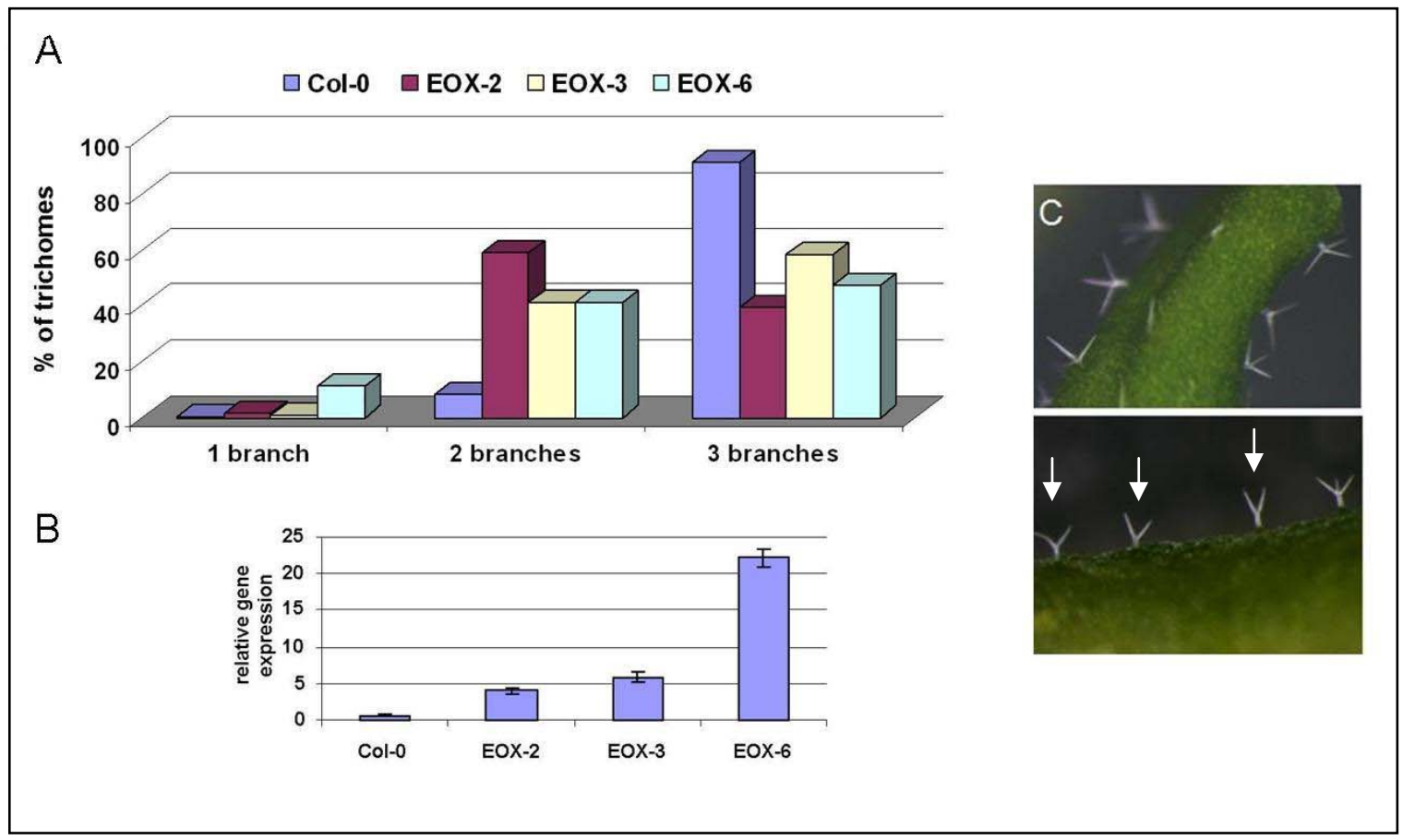

Figure 20: (A) Trichome branching in estradiol-inducible over-expressing lines (ER8AtRLCK VI_A2 lines). $\mathrm{T}_{1}$ plants were grown in the presence and sprayed with $10 \mu \mathrm{M}{ }^{17} \beta$ estradiol. Branch number was counted from isolated trichomes of the true leaves of three independent transgenic lines (EOX-2, 3 and 6). (A) Percentage of total trichomes is represented. (B) Relative expression level was determined in the lines analyzed by qRT-PCR. The expression level is determined as relative to non-induced control. GAPDH_2 was used as housekeeping gene for data normalization. (C) Trichomes of wild-type plants (upper photo) and EOX-2 line (lower photo).

The estradiol induced over-expressing AtRLCK VI_A2 plants have more trichomes with fewer branches then Arabidopsis Columbia plants grown in the presence of $10 \mu \mathrm{M}{ }^{17} \beta$-estradiol. Decreasing in the percentage of three-branched trichomes and increasing in the percentage of trichomes with one or two branches could be observed in all three transgenic lines. It is intriguing that the line 2 (EOX-2), which has the lowest 
over-expression level of transgene among the lines analyzed (Figure $20 \mathrm{~B}$ ), showed the highest increasing in the number of trichomes with 2 branches. However, over-expressing line-6 (EOX-6) which showed the highest transgene level after induction (Figure $20 \mathrm{~B}$ ) has more trichomes with 1 branch compared with the other two lines (Figure $20 \mathrm{~A}$ ).

The transgenic plants were checked in the absence of the inducer as well and it showed that the percentage of trichomes with fewer branches was somewhat increased compared to the Arabidopsis non-transformed plants, indicating that the promoter is leaky but in the induced lines the numbers of these types of trichomes was at least twothree-fold higher (data not shown).

In order to formulate a function for AtRLCK VI_A2 in this process, other lines have to be characterized and analized including the heat-inducible over-expressing AtRLCK VI_A2 plants.

Weather silencing of AtRLCK VI_A2 in plants have an effect on trichomes branching was also analyzed. The trichomes were isolated from $\mathrm{T}_{1}$ plants with silenced AtRLCK VI_A2 line7 at 3 weeks after germination. $T_{1}$ plants were germinated on selective media, sprayed every two days with $10 \mu \mathrm{M} 17 \beta$-estradiol, and kept at $25^{\circ} \mathrm{C}$ in a growth chamber. The decrease in the transgene level was determined by semiquantitative PCR at the time when trichomes were isolated (Figure $21 \mathrm{E}$ ).

In a preliminary analysis, the AtRLCK VI_A2 silencing transgenic plants did not exhibit a clear trichome phenotype except the appearance of trichomes with 5 branches (Figure 21 B, C, D) at a very low frequency. Five branched-trichomes were not seen in the controls, only in the induced RNAi line. Further experiments have to be completed to optimize the estradiol treatment and to include more silencing lines to validate the significance of this observation. 


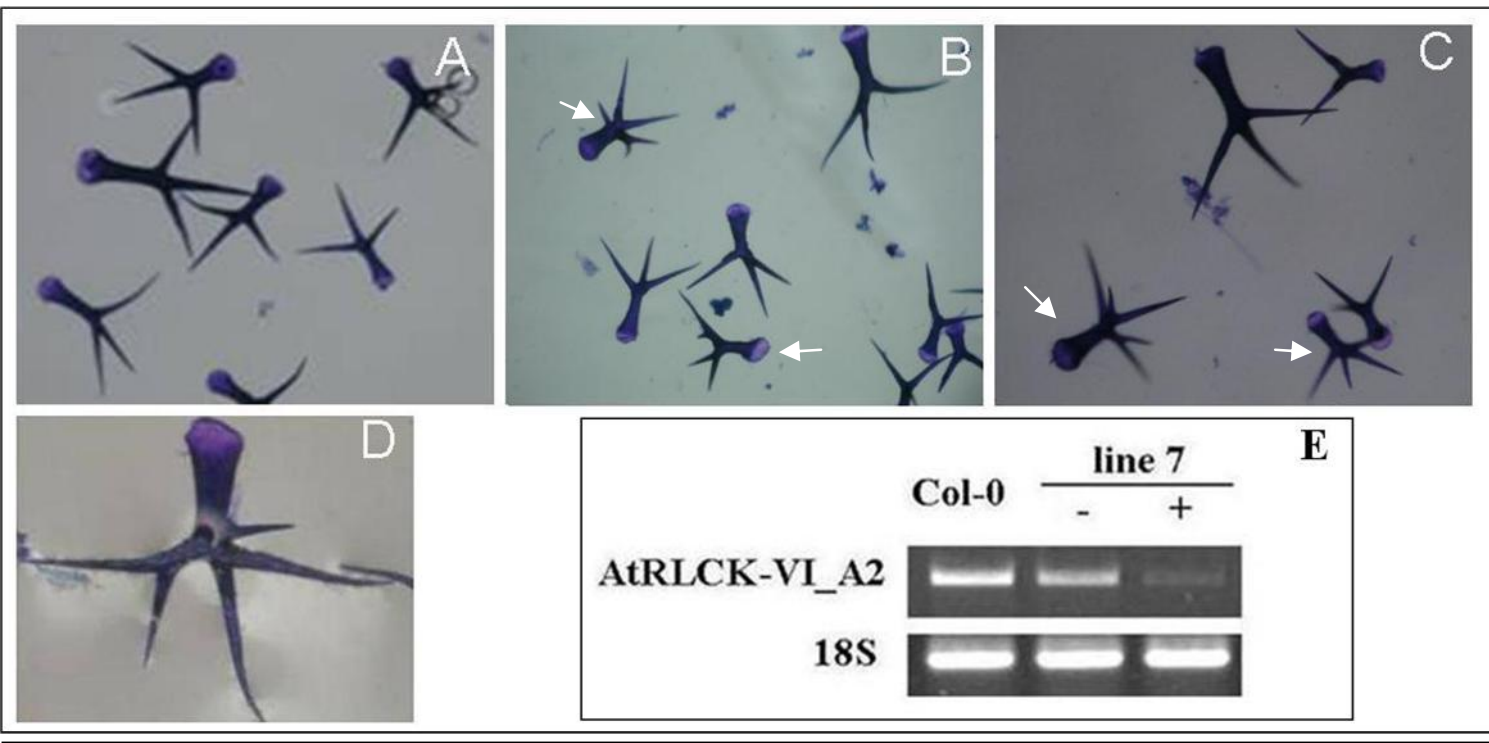

Figure 21: Inducibility of gene silencing and trichome branching in AtRLCK VI_A2 RNAi line7. Trichomes were isolated from true leaves of $\mathrm{T}_{1}$ plants grown in the presence and sprayed with $10 \mu \mathrm{M}{ }^{17} \beta$-estradiol. (A) Trichomes of the wild-type plants; (B), (C), (D) Trichomes of the RNAi plants, (E) Transgene expression level was determined in plants used in the experiment by semiquantitative PCR. As control primers those amplifying 18S rRNA.

Estradiol-inducible RNAi lines for other AtRLCK VI kinases were also used for the preliminary analysis and also showed the development of the trichomes with 5-6 branches after induction with estradiol (data not shown). This indicates that several RLCK VI members may be involved in trichome development and they may have redundant functions. Probably we have to switch off several kinases to have a stronger trichome phenotype.

Based on these data we can only conclude that over-expressing or silencing AtRLCK VI_A2 didn't show a strong modification in trichome morphogenesis, but this can be the reason for only a limited induction/reduction in the level of protein in trichomes or other unknown factors that need to be identified (estradiol concentration, more lines have to be checked). Heat-inducible overexpression of AtRLCK_A2 in transgenic lines remain also to be analyzed. 


\subsubsection{Over-expressed and silenced AtRLCK VI_A2 affects pollen tube growth}

Tip growth is another process dependent on ROP GTPase activity (Zheng and Yang 2000). To determine whether tip growth is affected in plants over-expressing or silencing the ROP-activated AtRLCK VI_A2 kinase, the growth of their pollen tubes was studied in the presence or absence of estradiol inducing transgene/RNAi expression. Homozygous plants were used in this experiment.

Pollen tubes from plants over-expressing AtRLCK VI_A2 appeared to be affected (Figure 22 B). In the case of these plants, bifurcated pollen tubes could frequently be observed. It seems that a new protrusion was organized, but growth at the original tip was also resumed, thus giving rise to double growth fronts.

The silencing of AtRLCK VI_A2 by RNA interference seemed to affect also the polarity of the pollen tube. Following a period of normal growth, polarity was often lost and a balloon-like tip developed on the pollen tube (Figure 22 C).

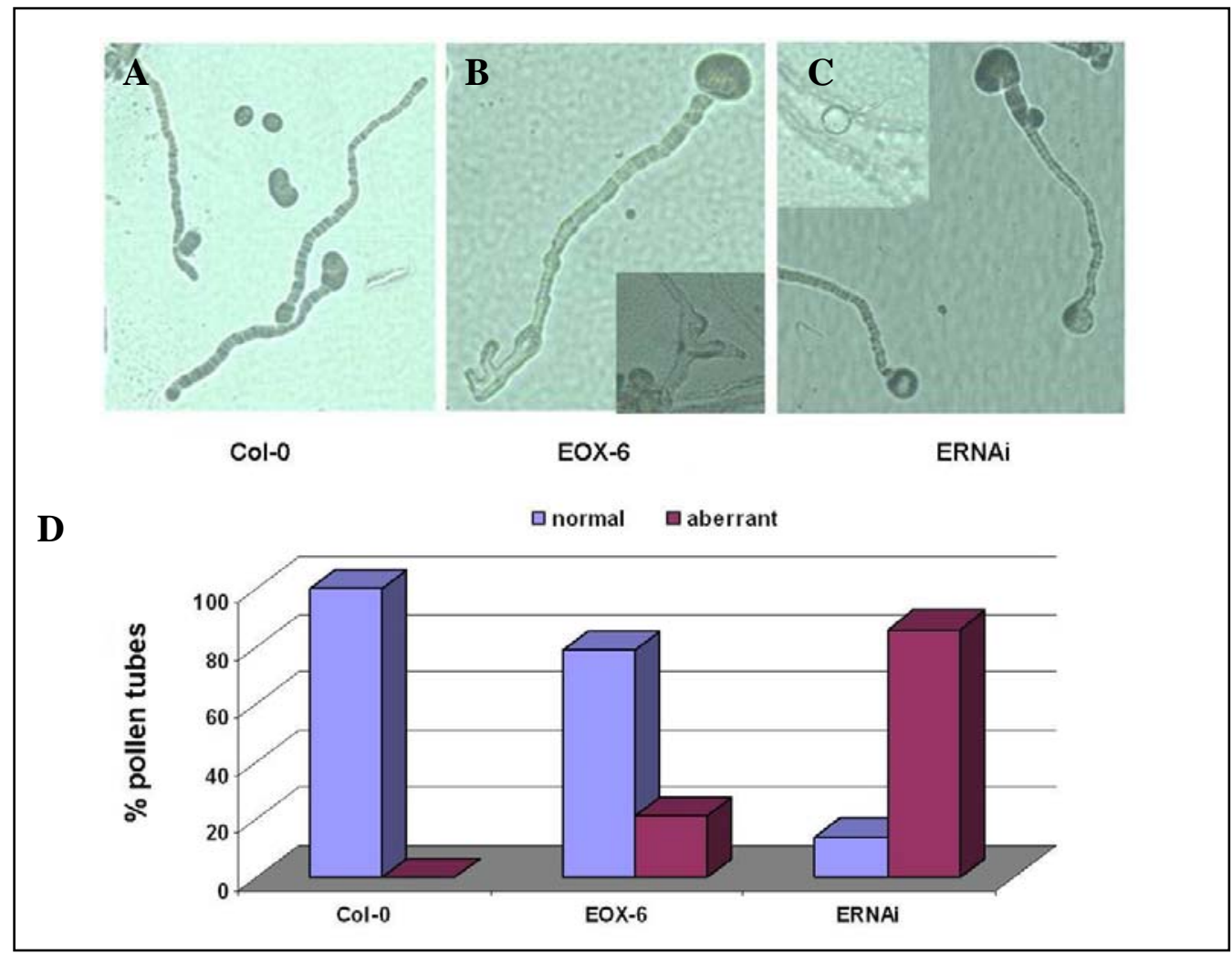

Figure 22: Over-expression and silencing AtRLCK VI_A2 in Arabidopsis plants affects pollen tube growth. Pollen tubes were grown in the presence of $10 \mu \mathrm{M}{ }^{17} \beta$-estradiol. (A) nontransformed Arabidopsis thalina Columbia ecotype; (B) ER8-AtRLCK VI_A2 homozygous overexpressing line; (C) ER8-RNAi-AtRLCK_A2 homozygous RNAi line; (D) Frequency of normal and aberrant pollen tubes from $\sim 25$ pollen tubes analyzed. 
Although, pollen tube growth was affected in tested lines, normal pollen tube growth could also be observed in both cases. Further experiments will include pollen tube growth on different estradiol concentrations as well as under different conditions of growth. Several other over-expressing and RNAi lines will also be checked. 


\section{Discussion and conclusions}

\subsection{In silico analysis of the primary structure of AtRLCK VI family members}

The RLK family, comprising more than 600 members in Arabidopsis, is a monophyletic group thought to have diverged specifically in the plant kingdom, and only related families of small size can be found in animals and in Plasmodium species (Shiu and Bleecker 2003). RLK subfamilies with different domain organizations are the results of ancient gene duplication events, which occurred extensively before the divergence of vascular and nonvascular plants, or even during the early evolution of streptophytes (Sasaki et al. 2007). In addition to RLK sequences, this monophyletic group also included kinases with no apparent signal sequence or transmembrane domain, and they were collectively named receptor-like cytoplasmic kinases (RLCKs). Some of these kinases formed subfamilies distinct from other RLKs, whereas others were embedded within several different RLK subfamilies.

The RLCK VI subfamily contains 14 proteins in Arabidopsis. The phylogenetic comparison of the Arabidopsis RLCK VI sequences revealed that the 14 proteins form two groups and within the groups there is a pair-wise similarity of certain sequences (Figure 6), indicating that gene duplication played a significant role in the formation of this protein family as well. Indeed, at least in four cases, the pairs of corresponding Arabidopsis genes (RLCK VI_A1/A2, B1/B2, B3/B4, and B6/B7) can be considered as paralogous genes residing within large duplicated chromosome regions (for the database of Arabidopsis paralogons see http:/www.wolfe.gen.tcd.ie/athal/dup). The duplication resulting in RLCK VI_A1/A2 likely represents an ancient polyploidization process (at least 100 million years ago) while the other duplication events leading to the formation of the paralogons RLCK VI_B1/B2, B3/B4, and B6/B7 can be dated back to about 20-24 million years ago (see http://www.wolfe.gen.tcd.ie/athal/dup; for more details see Blanc et al. 2003; Blanc and Wolfe 2004 b). Four large-scale duplications events in addition to lineage-specific expansions were supposed to contributed to the high RLK gene number present in Arabidopsis today (Vision et al. 2000; Shiu and Bleecker 2003; Shiu and Li 2004). 
The group A of Arabidopsis RLCK VI kinases is characterized by the presence of an $\mathrm{N}$-terminal serine-rich region (four out of the seven proteins) while several (four) group B members carry an N-terminal UspA domain (Kerk et al. 2003). The UspA domain is present either in small proteins, or it makes up the N-terminal portion of a larger protein, usually a protein kinase. In Arabidopsis, in addition to the RLCK VI members, some of the kinases belonging to the PERK ("plant external response like kinase" or proline-rich receptor-like kinase) and RLCK IX families as well as several small proteins carry the UspA sequence motif (Kerk et al. 2003). The UspA protein is an Escherichia coli protein that is highly expressed at the stationary phase of growth and mediates survival of the starved cells (Nystrom and Neidhardt 1994). The precise biological function of the UspA protein and the UspA domain is not know, however, it may be involved in nucleotide (ATP)-binding and signal transduction (Zarembinski et al. 1998). Although some of the small UspA-like proteins of Arabidopsis are implicated to play role in stress responses, nothing is know about the biological function of UspAcontaining kinases (Kerk et al. 2003). However, the UspA domains of the various Arabidopsis kinases and the small proteins are sufficiently divergent to indicate diverse functions (Kerk et al. 2003).

The RLCK VI_A1 protein possesses both (UspA and serine-rich) domains and may be the most closely related to the ancestor of this kinase family. It can be supposed that the domain structure of the kinases diverged following the gene duplication events.

\subsection{Gene expression profiling of AtRLCKs VI kinases based on a gene-specific real-time quantitative PCR approach}

Duplicated genes may follow various scenarios during evolution resulting in nonfunctionalization (silencing or null mutation), subfunctionalization (partitioning of functional modules of the ancestral gene), or neofunctionalization (gain of novel functions by at least one of the paralogs). Shifting in the expression pattern of the paralogous genes is one of the processes that can lead to non-, neo-, or subfunctionalization (Adams et al. 2003, Osborn et al. 2003). Expression divergence, resulting in rapid subfunctionalization, is suggested to contribute substantially to the maintenance of most of the paralogous regulatory and stress-responsive genes in the 
Arabidopsis genome (Haberer et al. 2004, Blanc and Wolfe 2004, Kim et al. 2005, Duarte et al. 2006, Walther et al. 2007).

In agreement with this hypothesis, our gene expression data verified a diverse expression pattern of the 14 investigated RLCK VI genes, in the various plant organs as well as in response to stress/hormone treatments. In certain cases, the paralogous genes retained a similar relative expression pattern while in other cases (e.g. RLCK VI_A6 and A7) they exhibited complementarities in this respect (e.g. RLCK VI_A3 and A4). Paralogons, which derive from large-scale duplication events and can still be found in duplicated segments, exhibit more correlated expression patterns as compared to those resulting from small-scale duplications or those residing no longer on duplicated segments (Casneuf et al. 2006). This may explain the difference between RLCK VI group A and group B considering the breadth of their gene expression diversity, since group B genes (at least six out of the seven) reside in large duplicated regions of recent duplications and exhibit only limited differences in their relative transcript levels.

The presented data indicate that the activity of several members of the RLCK VI kinase subfamily is regulated at the transcriptional level during plant development as well as in response to environmental stresses. Their tightly regulated and generally low expression may indicate that these proteins are involved in specific rather than general cellular processes. The low expression levels in dedifferentiated cultured cells and diverse distribution in various plant organs suggest that their functions are associated with plant differentiation. In addition, some of them may also be involved in the coordination of plant development and stress responses.

\subsection{Co-expression study of RLCKs and other ROP GTPase effectors and regulators based on in silico data analysis}

Plant RhoGTPases (ROPs) mediate multiple extracellular signals ranging from hormone to stress and regulate diverse cellular processes important for polarized cell growth, differentiation, development, reproduction, and responses to the environment. ROP GTPases have the potential to interact with a plethora of regulators and effectors that finally determines their signaling specificity. There were indirect indications that GTPase- and kinase-mediated signaling pathways are interlinked in plants (Lieberherr et al. 2005, Molendijk et al. 2008, Dorjgotov et al. 2009, Kost et al. 
1999). Silencing of the small GTPase OsRacl by RNA interference prevented the elicitor-induced accumulation and posttranslational activation of the rice kinase OsMAPK6, but the mechanism how the activation take place is still not known (Lieberherr et al. 2005). However, recently in our group, kinases directly activated by plant Rho-type (ROP) were identified (Dorjgotov et al. 2009). A direct interaction of plant ROP GTPases with receptor-like cytoplasmic kinases (RLCK VI_A4 and A6) has been reported in Arabidopsis as well as in alfalfa (Medicago truncatula RLCK VI_A4 and A6 (Molendijk et al. 2008; Dorjgotov et al. 2009), and indicated that RLCK VI kinases may serve as ROP GTPase effectors. We showed that members of the Medicago and Arabidopsis RLCK VI family are specifically activated by ROP GTPases (Dorjgotov et al. 2009).

In order to strengthen the view that RLCK VI members may indeed serve as ROP effectors in planta, the co-expression of ROPs and RLCK VI_A kinases was analysed in silico based on microarray data sets. Moreover, to have a full picture on potential partners involved in given ROP-dependent signaling pathways all known ROP regulators and effectors were included into the study.

The hierarchical clustering analysis of microarray expression data showed clear correlation of the relative expression pattern of several of the investigated proteins with organ/tissue specific expression while in the case of others the expression in several organs/tissues made it difficult to predict functional interactions among them.

Several AtRLCK VI_A kinases (A3, A6 and A7) belongs to group of genes with high and specific expression in pollen (Figure 10). The cluster contains several AtROPs, regulators of ROP GTPases as well as effectors proteins as RIC and two NADPH complex subunits. The role of ROP GTPases and their regulators and effectors in pollen tube growth is well established. Pollen-specific expression ROP1,3,5 GTPases is well demonstrated ( $\mathrm{Li}$ et al. 1998) and the specific expression of a subclass of ROPGEFs in the pollen has also been confirmed by RT-PCR analysis (Zhang and McCormick 2007). Abundance of these transcripts in the pollen is mainly related to the establishment of polarity during pollen tube growth (Kost 2008; Fu and Yang 2001; Cole and Fowler 2006). The representation of the various families with several members indicates the significance of the process the success of which is ensured by parallel pathways. 
Our qRT-PCR data in relation to the gene expression in the Arabidopsis organs also showed that AtRLCK VI_A3, A6 and A7 are expressed in flower buds and open flowers. Although RLCK VI_A1 also belongs to this cluster due to its overall gene expression pattern (Figure 10), its expression is much weaker in the pollen (Figure 7).

Another AtRLCK VI member, A4 was found out to belong to a group of genes with high expression in root, among many NADPH complex subunits. In our qRT-PCR gene expression analysis the RLCK VI_A4 as well B3 and B4 genes were expressed at a level above average in the root. Increased expression of AtRLCK VI_A4 in the rosette and /or cauline leaves could be observed as well. The NADPH oxidase is required for root hair tip growth. In Arabidopis AtRBOHC-deficient $r h d 2$ mutants have short root hairs and stunted roots as consequences of defective ROS production, $\mathrm{Ca} 2+$ uptake and consequently cell expansion (Foreman et al. 2003). Moreover, it was suggested that NADPH oxidase activity required for root hair formation is under the control of ROP/RAC GTPases. Root epidermal cells of Arabidopsis scnl mutants missing a RhoGDI isoform are unable to polarize ROP activity and consequently fail to form normal root hairs and ROS production is also enhanced and depolarized (Carol et al. 2005). AtROP2, AtROP4 and 6 are known to play role in root hair growth (Jones et al. 2002, Molendijk et al. 2001) and a functional relation between AtROP2 and AtRBOHC was indicated (Fu et al. 2001). AtROP 2, 4 and 6 are not specific expressed in roots, they shows a more ubiquitous expression pattern (Figure 10).

AtRLCK VI_A5, form a specific group with high relative expression in stems, nodes and hypocotyls, together with RIC4, ROP7, GAP5, RIC2 (Figure 10) implicating the role of ROP-signaling pathways in the development of these organs. Several other genes from RLCK class VI exhibited above average expression in the inflorescence stem in our qRT-PCR data and AtRLCK VI_A5 showed the highest expression in this organ.

This gene expression analysis is a complementary approach to indicate the biological specificity and functional potential of predicted interactions. Only few potential interactions indicated by the characteristic gene expression clusters on Figure 10 has already been experimentally validated such as the interaction of the preferentially pollen expressed AtROP1 with RIC1, 4 and GEF9 as well as NtRAC5 (ROP5) with GDI2a, in Arabidopsis and tobacco, respectively (see Introduction Tables 1 and 2). In 
contrast, the functional interaction of ROPs and ROP partners with distinct expression patterns could also be demonstrated for example in epidermal cells (AtROP2, 4 and AtRIC1, 4 (Fu et al. 2005)) where these proteins are expressed only at a low level in comparison to other organs (Figure 10).

There are several studies which indicate the coevolution of interacting proteins and members of signaling networks preserving similar expression pattern of the involved genes in order to ensure the presence of the partners in proper ratios (Ettwiller and Veitia, 2007; Fraser et al. 2004).

\subsection{RLCK-ROP GTPase interaction specificity}

Yeast two hybrid interactions between different AtROPs and AtRLCKs, confirmed that the interaction is specific for the group A of the Arabidopsis RLCK VI family (with the exception of the AtRLCK VI_A7 kinase from presently unknown reasons) while representants of the RLCK IV, VII and IX families included in the experiment showed no ROP-binding. However, the recently published interaction of the AtROP4 and AtROP11 GTPases with an RLCK Class VIII kinase (NRCK, Molendijk et al. 2009) suggests that the capability of RLCKs to bind ROP GTPases is not limited to the RLCK VI group A. Based on this observation and considering the high number of RLCK kinases in plants (twelve families with 193 members in Arabidopsis) the existence of further ROP binding RLCKs can be hypothesized.

Interestingly, none of the ROP-interacting RLCK kinases has any recognizable sequence motif known to play role in the binding of Rho-type GTPases to their effectors (Karnoub et al. 2004; Berken and Wittinghofer 2008; Burbelo et al. 1995).

In yeast and animal cells it is well known that Rho-associated kinases are an important class of Rho effectors. Rho GTPases commonly bind to conserved binding domains outside the catalytic domain of their target kinases (Zhao and Manser 2005). The first kinases identified as Rho GTPase regulated ones were the "p21 protein activated" or PAK kinases. These kinases are known to bind specifically to the GTP-bound CDC42/RAC GTPase (Hofmann et al. 2004; Leveleki et al. 2004; Mahlert et al. 2006). PAK kinases have a CRIB (CDC42/Rac-interactive binding) motif, which is responsible for the interaction with GTPases. In animal cells, the CRIB motif (also known as PBD or p21-binding domain) is used for GTP-dependent interaction with Rac and Cdc2 
subfamily members by a number of Rho effector proteins. In plants, a similar CRIB-like motive is present in plant RopGAPs and the small ROP-effector scaffold proteins RICs (ROP-interacting CRIB-containing proteins) which regulate cytoskeletal dynamics (Fu et al. 2005; Wu et al. 2000, 2001). However, none of the plant kinases, including the ROPinteracting RLCK kinases, have any characteristic domain or motif that could be correlated with their ability to bind ROP GTPases. In Arabidopsis (Molendijk et al. 2008) and we reported in Medicago (Dorjgotov et al. 2009) that the interaction with ROPs can tolerate only the deletion of short $\mathrm{N}$ - or C-terminal RLCK sequences. We supposed that not a single well-defined domain but several residues/motives on the surface of the kinases may be involved in ROP binding (Dorjgotov et al. 2009). The interaction of RLCKs with ROPs may be based on a presently unknown mechanism or structural feature that needs further investigations.

In an alignment that included all 14 members of the AtRLCK VI family consisting of the ROP-interacting RLCK VI_A and the non-interacting RLCK VI_B groups, three further kinases that belong to RLCK Class VII and VIII (NAK, Pti1) and a mitogen-activated protein kinases (AtMPK1) we could observed several characteristic differences in the sequences of the kinases belonging to group A that showed interaction with ROPs in yeast two hybrid assays. Several residues/motives could be identified that may have a role in the RLCK-ROP binding (Figure 12).

The AtRLCK VI_A7 is a member of RLCK VI group A that could not interact with any ROP GTPase in the yeast two hybrid assay, although we could show by western blot that the protein was expressed in the yeast cells. From the comparison of the primary sequences, we could not find out what is the reason for this. Those regions that are characteristic for AtRLCK VI group A members in comparison to those belonging to the group B are also conserved in AtRLCK VI_A7. The only characteristic differences that this kinase has a N-terminal His-rich domain, but at present nothing is known about the potential function of this region. A possible explanation is that it might have only a transient or week interaction that is difficult to detect by the yeast two hybrid assay. It is also possible that the protein was not properly folded (e.g. due to the His-rich domain) or other factors in the yeast cell (yeast cells also have Rho GTPases) might also affect the 
affinity between the proteins. Further experiments could only answer the question whether AtRLCK VI_7 is a real exception within the group in respect of ROP interaction.

Primary sequence analysis revealed that some of the members of the RLCK VI family have short membrane attachement sites or transmembrane spanning region (Figure 6), which might be relevant to connect them to specific membrane domains together with ROPs. For example, AtRLCK VI_A5 kinase posses a short N-terminal hydrophobic region but we have no data about the role of this region in membrane binding.

Cellular distribution of these kinases must be relevant also to connect them to specific ROPs. We have not made experiments to determine the cellular localization of Arabidopsis RLCK VI kinases. Molendijk and co-workers has shown the mostly cytoplasmic localization for YFP-tagged AtRLCK VI_A4 (Molendijk et al. 2008), and in our laboratory we have also shown (unpublished data) for two Medicago RLCK VI kinases, the homologs of AtRLCK VI_A2 and A5, that they are cytoplasmically localized. Active ROP GTPases are also membrane localized therefore the ROP-kinase interaction has to take place there. Indeed, when RLCKs are co-expressed together with CA-ROP GTPase they are targeted to the plasma membrane in transformed protoplasts as we could show it for the Medicago RLCKs (unpublished data) and Molendijk and co-workers for the Arabidopsis RLCK VI_A4 (Molendijk et al. 2008).

In in vitro pull down assays, both AtRLCK VI_A2 and AtRLCK VI_B3 could bind to all conformations of AtROP1. Moreover in our laboratory, a pull down assay with MtRRK1 (homolog of Arabidopsis AtRLCK VI_A2) and MtROP6 showed that the kinase could bind also both the DN or GDP-bound form of the GTPase but exhibited a higher affinity for CA or GTP-bound GTPase (Dorjgotov et al. 2009). Molendijk et al. (2008) reported similar result. They verified protein-protein interaction of AtRLCKs and AtROPs in transfected Arabidopsis protoplasts using bimolecular fluorescence complementation (BiFC) assay. In those experiments, RLCK kinases could also form complexes with ROP GTPases in all conformations but with a preference for the GTPbound form.

In pull down assays, the experimental conditions (protein quantity, salt, detergents, blocking agents) influence the interaction between two proteins. Moreover, the environment might restrict mobility and therefore enhance the apparent affinity of an 
interaction or the proteins might not be properly folded in these conditions and an artificial interaction might result from the exposure of hydrophobic surfaces.

The two-hybrid system is an in vivo assay, and proteins are more likely to be in their native conformation compared to a pull down assay. Moreover, the conditions are more uniform in yeast two hybrid assays and therefore these tests are more reproducible as compared to in vitro pull down assays.

Therefore we can conclude that the differences in the results obtained by the various protein-protein interaction approaches may be related to the differing conditions in vitro, in yeast or in planta stabilizing or weekening the interactions.

However, inspite of the potential interactions of ROPs with various RLCK kinases in vitro, the kinase activity assays clearly indicated specificity towards a functional interaction of ROPs only with the RLCK VI_A kinases.

\subsection{In vitro activation of RLCK_VI kinases by ROP GTPases}

We found that AtRLCK VI_A2 could be activated in vitro by AtROP1 as well as MtROP6 GTPases and that the presence of the GTPases in the GTP-bound conformation was the most efficient in increasing the phosphorylation activity of the kinase. The data are in agreement with those obtained on Medicago RRK kinases and ROP GTPases (Dorjgotov et al. 2009).

The GTP-binding capability of ROPs as well as the activity of the kinases was dependent on the protein purification therefore there were variations in the degree of activation from experiment to experiment. Moreover, in the in vitro kinase assays we could not determine the actual ratio of active/inactive (GTP/GDP-bound) GTPases, although we have used the same molar ratio of kinases and G-proteins. AtRLCK VI_A6/RBK2 was shown to be strongly activated by the CA-form of the AtROP1 GTPase. The CA ROP GTPase is stabilized in the active conformation and therefore the interaction is independent of the binding efficiency between the nucleotide and the ROP GTPase.

AtRLCK VI_B3 and an AtRLCK from class VII could not be activated by AtROP1 indicating specificity towards RLCK VI_A kinases. Based on the fact that RLCK VI_A kinases are preferentially and strongly activated by the GTPase in active conformation, it could be hypothesize that these kinases are potential downstream ROP 
GTPase effectors. This possibility was disapproved by Molendijk et al. (2008) first reporting ROP-RLCK interactions. They could not observe ROP GTPase-dependent in vitro autophosphorylation or AtROP4 substrate phosphorylation changes with ROPinteracting RLCK kinases immunoprecipitated from insect cells where they were coexpressed.

Although it is likely that RLCK VI_A kinases serve as ROP effectors, it is not clear how the GTPases can activate the kinases. RLCKs do not have any recognizable regulatory region implicated in the Rho GTPase-dependent activation of certain yeast and animal kinases. Only future investigations of various mutant kinase forms and especially the determination of the structure of the active ROP-RLCK VI_A complex can give clues to understand ROP-mediated RLCK activation.

We know a bit more as the region of the ROP GTPase involved in kinase activation is considered. A specific region of RhoGTPases, the so called Rho-insert region, is implicated in effector binding and activation (Karnoub et al. 2004). Plant ROP GTPases have an insert region with characteristic differences as compared to human or yeast Rac, Rho ad Cdc42 proteins that is an indication that plants have evolved specific ROP GTPase effectors (Berken, 2008). In our laboratory it was previously demonstrated that this region is important for the activation of the MtRRK1 (MtRLCK VI_A2) kinase (Dorjgotov et al. 2009).

\subsection{Over-expressing and silencing AtRLCK VI_A2 induced modification in trichome branching and pollen tube growth}

Cell polarization is intimately linked to plant development, growth, and responses to the environment. It is well established that plant cells use conserved mechanisms such as Rho family GTPases (ROPs) to integrate both plant-specific and conserved polarity cues and to coordinate the cytoskeketon dynamics/reorganization and vesicular trafficking required for polarity establishment and maintenance. Our data showed that kinases belonging to the RLCK Class VI Arabidopsis thaliana can be specifically activated by GTP-bound ROP GTPases in vitro further supporting the view that plant ROP GTPases may directly regulate downstream kinase signaling. In this direction, we proceeded with analysis of transgenic plant over-expressing and silencing AtRLCK VI_A2. We suspect that AtRLCK VI_A2 as a potential ROP effector may have a role in 
polarity establishment as well. Directional cell expansion (e.g. trichomes) and tip growth (e.g. pollen tubes) are two basic processes underlying the morphogenesis of polar cell types in plants. Therefore these two processes were first investigated during the preliminary characterization of transgenic Arabidopsis lines over-expressing or silencing the gene coding for the AtRLCK VI_A2 kinase. These preliminary data that need further confirmation indicated that both over-expressing as well as silencing of the gene disturb polar growth of the pollen tube and might affect trichome branching.

Over-expression of AtRLCK VI_A2 increase the frequency of trichomes with fewer branches while silencing of the gene may lead to the apparition of trichomes with more branches, although only with a low frequency. A large number of trichome branching mutants have already been identified in which the number of branches is either increased or reduced (Folkers et al. 1997; Luo and Oppenheimer 1999). Generally, mutants with altered ploidy levels are also affected in branch number such that mutants with a reduced DNA content have fewer branches and mutants with increased ploidy levels have more branches (Hulskamp et al. 1994; Folkers et al. 1997). This correlation between ploidy level and branch number indicates that either the size or the growth time is relevant for the initiation of branches. The analysis of drug-treated trichomes and mutant analysis both point to the microtubules (MT) as an important regulator of branch initiation. Stabilizing the MT increases the branch number of wild type trichomes and induces branching of unbranched mutant trichomes, whereas MT disruption causes unbranched trichomes (Mathur and Chua 2000). The role of ROP GTPase-mediated signaling in trichome morphogenesis via the regulation of cytoskeleton organization (microtubules as well as microfilaments) is well established (Mathur 2006).

Over-expressing AtRLCK VI_A2 in the pollen tube may result in the formation of bifurcated pollen tube tip. In Arabidopsis, over-expressing AtROPs in pollen tubes showed a range of pollen tube defects (Cheung et al. 2003). Among others, bifurcated pollen tubes could be observed by over-expressing AtROP9 (Cheung et al. 2003). Therefore, this phenotype caused by kinase overexpression may also be related to the link of the kinase to ROP signaling.

Silencing of AtRLCK VI_A2 could lead to the formation of ballooned pollen tube tips. The development of ballooned tip on growing pollen tubes can also be the result of 
ectopic ROP expression (Chen et al. 2003; Cheung et al. 2003; Kost et al. 1999; Speranza et al. 2001). The cylindrical shape of normal pollen tubes is a result of polarized growth that is restricted to the apical region of the tubes (Speranza et al. 2001). Ballooning is the result of the presence and activation of the GTPase outside of the tip region resulting in the loss of polarity. The balloon tip morphology detected in pollen tubes silencing AtRLCK VI_A2 implies that growth is no longer restricted to the apical region. This phenotype may indicate that there is a negative feed back regulation of the ROP effector kinase on ROP activation restricting it to the tip region.

In pollen tubes over-expressing ROP GTPases, a network of irregular actin cables was detected in the apical region (Fu et al. 2001; Kost et al. 1999), indicating that an aberrant arrangement of F-actin in the tip region caused de-polarized growth at the ballon tip morphology. The observed pollen tube phenotypes were restricted only to a subset of pollen tubes and tubes which showed normal growth could also been observed even in homozygous plants. This phenomenon can be due to the uneven induction of the transgene by the estradiol treatment. The severity of pollen tube defects might be dependent on the expression level of the protein in individual tubes.

Further more detailed investigations will be carried out to confirm the potential role of RLCK VI_A kinases in these and further ROP GTPase-regulated processes (e.g. pathogene response, ROS production) to support the view that RLCKs are indeed plant specific ROP GTPase effector kinases. Towards this goal, we have collected and produced several kinase mutants and transgenics including all RLCK VI_A subfamily members. The primary molecular characterization of these plants is underway now. Furthermore a transient gene expression system in tobacco pollen tubes was established in collaboration with the laboratory of Prof. Victor Zarsky (Charles University, Prague) that will allow a fast analysis the ectopic expression of the kinases with and without the coexpression of various GTPase forms on polar growth.

In conclusion, we established that AtRLCK VI_A kinases interact and are activated in vitro by AtROP GTPases. Furthermore, the characterization of expression patterns and transgenic plants allowed us to hypothesize that these kinases may serve ROP GTPase effectors in plants. This signaling step has several plant-specific aspects, 
further studies of which will provide interesting insight into the evolution of the cellular signaling as well as valuable information about the regulation of plant development. 


\section{SUMMARY}

Plants have to respond and adapt to a variety of continuously changing environmental factors in order to establish an appropriate developmental strategy to ensure survival. There are ample data showing that protein phosphorylation/dephosphorylation plays a central role in cellular signal transduction in all organisms (Herrmann et al. 2006; Stone and Walker 1995).

Interestingly, plants have a similar system as mammals to detect and transfer signals across the cell membrane into the nucleus where adaptations could be initiated. For the detection and transfer of an external signal, mammalian systems have receptor protein kinases. The proteins contain a single hydrophobic transmembrane domain, an extracellular domain and protein kinase domain. The majority of animal receptor kinases are phosphorylated on tyrosine residues within the kinase domain (receptor tyrosin kinases or RTKs; Ullrich and Schlessinger 1990), but a few were discovered which are phosphorylated on serine and threonine residues (Lin et al. 1992).

In plants, two different types of transmembrane receptor kinases are known, including receptor-like serine/threonine (Ser/Thr) kinases (receptor-like kinases RLKs; Shiu and Bleecker 2001, 2003; Shiu et al. 2004; Walker 1994), structurally similar to mammalian RTKs, and receptor histidine (His) kinases (Grefen and Harter 2004; Mizuno 2005; Urao et al. 2000).

Since the first RLK-encoding gene family was found in Zea mays (Walker and Zhang 1990), thousands of RLK genes have been identified from many different plant species. The Arabidopsis genome contains more than 600 members, representing nearly $2.5 \%$ of the annotated protein-coding genes; and more than 1000 members were annotated in the rice genome (Shiu et al. 2004).

Approximately $25 \%$ of the Arabidopsis RLKs contain only a kinase domain with no apparent signal sequence or transmembrane region and thus were collectively named as receptor like cytoplasmic kinases (RLCKs). Arabidopsis RLCKs can be subdivided into 12 classes with 193 protein coding genes all together.

Concerning the function of plant RLCKs, at the present only few members have been characterized and it is very likely that they play major role in the perception and 
transmission of external signals perceived by RLKs (Zhou et al. 1995; Murase et al. 2004).

Recently, our group as well as a group in Germany reported a direct interaction of plant ROP GTPases with receptor-like cytoplasmic kinases (RLCK class VI) from Arabidopsis (Molendijk et al. 2008) and alfalfa (Dorjgotov et al. 2009). Moreover, we provide evidences that kinases belonging to the RLCK Class VI family of Medicago truncatula and Arabidopsis thaliana can be specifically activated by GTP-bound ROP GTPases in vitro further supporting the view that plant Rho (ROP) G-proteins may directly regulate downstream kinase signaling.

A further kinase designated as cysteine-rich receptor kinase (NCRK) belonging to a distinct kinase family has also been shown to interact with ROPs (Molendijk et al. 2008). None of these plant specific ROP-interacting kinases has any characteristic domain or motif that could be correlated with their ability to bind ROP GTPases.

Plant specific ROP GTPases are versatile molecular switches in many processes during plant growth, development and responses to the environment and thus a possible implication of RLCKs in these ROP-dependent signal transduction pathways is in discussion.

Our general aim was to characterize the members of the Arabidopsis thaliana RLCK Class VI family of protein kinases. Despite of their potential significance in ROP GTPase mediated signaling, hardly any functional information was available until now about the fourteen Arabidopsis RLCK Class VI members.

Sequence comparison and phylogenetic analysis revealed that gene duplication played a significant role in the formation of this kinase family and allowed the separation of the 14 RCLK VI kinases into two groups with seven members each (A1 to A7 and B1 to B7). The proteins are highly homologous to each other, especially in the kinase domain, but are divergent from the related kinase families. It was established that, several members have an N-terminal UspA ("universal stress protein") domain (group B members) or an N-terminal serine-rich region (group A members).

In order to formulate a possible biological role of AtRLCK_VI kinases, real-time quantitative reverse transcription-polymerase reaction (qRT-PCR) was used to determine relative transcript levels in the various organs (root, rosette leaves, cauline leaves, 
inflorescence stem, flower buds, open flowers, siliques. exponentially dividing cultured cells) of the Arabidopsis plant as well as under a series of abiotic stress/hormone (osmotic, sugar, salt stress, oxidative stress, cold and hormone treatment) treatments in seedlings.

AtRLCK VI genes exhibited diverse expression patterns in the various plant organs as well as in response to stress/hormone treatments. In certain cases, the paralogous genes retained a similar relative expression pattern. The presented data indicate that the activity of several members of the RLCK VI kinase subfamily is regulated at the transcriptional level during plant development as well as in response to environmental stresses. Their tightly regulated and generally low expression may indicate that these proteins are involved in specific rather than general cellular processes.

As it was mentioned above, we showed that members of the Medicago and Arabidopsis RLCK VI family are specifically activated by ROP GTPases (Dorjgotov et al. 2009). In order to strengthen the view that RLCK VI members may indeed serve as ROP effectors in planta, the co-expression of ROPs and RLCK VI_A kinases was analysed in silico based on microarray data sets. Moreover, to have a full picture on potential partners involved in given ROP-dependent signaling pathways, all known ROP regulators and effectors were included into the study.

The hierarchical clustering analysis of microarray expression data showed clear correlation of the relative expression pattern of several of the investigated proteins with organ/tissue specific expression. Five main expression clusters (A-E) could be established. These clusters include genes with preferential or more abundant expression in the pollen and flower; stem, node and hypocotyl; root; shoot and root tip; flower and silique; and all over the plant.

In order to test RLCK-ROP interaction specificity a yeast two hybrid interaction matrix was established using ten RLCK VI kinases (5-5 from groups A and B, respectively) and eight Arabidopsis ROP GTPases. The interaction was found to be specific for the RLCK VI group A kinases only. However, in an in vitro pull down assay a randomly selected kinase from the group B showed also interaction with a ROP GTPase. This difference in the results obtained by the various protein-protein interaction approaches may be related to the differing conditions stabilizing or weakening the 
interaction. However, based on the in vitro kinase activity assays (see further), we suppose that the interaction pattern obtained in the yeast assay is more specific and can better simulate the in planta conditions.

Primary sequence analysis revealed several characteristic differences in the sequences of the kinases belonging to group A that showed interaction with ROPs in yeast two hybrid assays. In this way several residues/motives could be identified that may have a role in the AtRLCK-ROP binding.

Inspite of the potential interactions of ROPs with various RLCK kinases in vitro, the kinase activity assays clearly indicated specificity towards a functional interaction between ROPs and only the A group of RLCK VI kinases. One kinase which belongs to the group B (AtRLCK VI_B3) and an other one from RLCK class VII, that were also tested, could not be activated by the AtROP1 GTPase in contrast to several RLCKVI_A members indicating ROP's specificity towards RLCK VI_A kinases. The presence of the GTPases in the GTP-bound conformation is the most efficient in increasing the phosphorylation activity of the kinase. Based on the fact that RLCK VI_A kinases are preferentially and strongly activated by the GTPase in active conformation, it could be hypothesized that these kinases are potential downstream ROP GTPase effectors. This possibility was disapproved by Molendijk et al. (2008) first reporting ROP-RLCK interactions. They could not observe ROP GTPase-dependent in vitro autophosphorylation or AtROP4 substrate phosphorylation with ROP-interacting RLCK kinases immunoprecipitated from insect cells where they were co-expressed.

Although it is likely that RLCK VI_A kinases serve as ROP effectors, it is not clear how the GTPases can activate the kinases. We know a bit more as the region of the ROP GTPase involved in kinase activation is considered. A specific region of RhoGTPases, the so called Rho-insert region, is implicated in effector binding and activation (Karnoub et al. 2004). Plant ROP GTPases have an insert region with characteristic differences as compared to human or yeast Rac, Rho and Cdc42 proteins that is an indication that plants have evolved specific ROP GTPase effectors (Berken, 2008). In our laboratory it was identified that this region is important for the activation of the MtRRK1 (MtRLCK VI_A2) kinase (Dorjgotov et al. 2009). 
The biological role of functional ROP-RLCK interaction needs to be determined. In this direction we further proceed with the production of transgenic plants overexpressing or silencing the RLCK VI_A2 gene.

Cell polarization is intimately linked to plant development, growth, and responses to the environment. It is well established that plant cells use conserved mechanisms such as Rho family GTPases (ROPs) to integrate both plant-specific and conserved polarity cues and to coordinate the cytoskeleton dynamics/reorganization and vesicular trafficking required for polarity establishment and maintenance.

We suspect that AtRLCK VI_A2 as a potential ROP effector may have a role in polarity establishment. Directional cell expansion (e.g. trichomes) and tip growth (e.g. pollen tubes) are two basic processes underlying the morphogenesis of polar cell types in plants.

Our preliminary data, that need further confirmation, indicated that both overexpressing as well as silencing of the gene disturb polar growth of the pollen tube and might affect trichome branching. Over-expression of AtRLCK VI_A2 increase the frequency of trichomes with fewer branches while silencing of the gene may lead to the apparition of trichomes with more branches, although only with a low frequency. Overexpressing AtRLCK VI_A2 in the pollen tube may result in the formation of bifurcated pollen tube tip. Silencing of AtRLCK VI_A2 could lead to the formation of ballooned pollen tube tips. Further more detailed investigations will be carried out to confirm the potential role of RLCK VI_A kinases in these and further ROP GTPase-regulated processes (e.g. pathogene response, ROS production) to support the view that RLCKs are indeed plant specific ROP GTPase effector kinases.

In summary, we established that AtRLCK VI_A kinases interact and are activated in vitro by AtROP GTPases. Furthermore, the characterization of expression patterns and transgenic plants allowed us to hypothesize that these kinases may serve as ROP GTPase effectors in plants. This signaling step has several plant-specific aspects. Further studies will allow interesting insights into the evolution of cellular signaling mechanisms and will provide valuable new information about the regulation of plant development. 


\section{8. ÖSSZEFOGLALÁS}

Ahhoz, hogy a növények megfelelő fejlődési stratégiát alakíthassanak ki a túlélés biztosítása érdekében, a folytonosan változó környezeti tényezőket érzékelniük kell és azokra megfelelő adaptív választ kell adniuk. Számos adat áll rendelkezésre amelyek azt bizonyítják, hogy a fehérjék foszforilációja/defoszforilációja a növények egyedfejlődésének és stressz válaszának szabályozásában központi szerepet játszik (Herrmann és mtsai. 2006; Stone és Walker 1995).

A növényi és az állati sejtek hasonló módon érzékelik és továbbítják a környezetből érkező jeleket a sejtmembránon át a sejtmagba. Az állati sejtek a külső jel érzékeléséhez és továbbításához a sejtmembránba ágyazott receptor protein kinázokat használnak. Ezek a fehérjék egy hidrofób transzmembrán doménnel, egy extracelluláris és egy kináz doménnel rendelkeznek. Az állatok receptor kinázainak nagy része tirozin aminosavat képes foszforilálni a szubsztráton (receptor tirozin kinázok vagy RTK; Ullrich és Schlessinger 1990), de néhányukról úgy találták, hogy szerin és treonin aminosavakat foszforilálnak (Lin és mtsai. 1992).

A növényekben két különböző típusú transzmembrán receptor kináz ismert, úgymind a szerkezetükben az emlősök RTK-jához hasonló receptor-szerű szerin/treonin (Ser/thr) kinázok (receptor-szerü kinázok, RLK; Shiu és Bleecker 2001, 2003; Shiu és mtsai. 2004; Walker 1994), valamint a receptor hisztidin (His) kinázok (Grefen és Harter 2004; Mizuno 2005; Urao és mtsai. 2000).

Azóta, hogy az első RLK-kat kódoló géncsaládot megtalálták kukoricában (Walker és Zhang 1990), RLK gének ezreit sikerült azonosítani számos különböző növényfajból. Az Arabidopsis genom több mint 600 RLK-t kódoló gént tartalmaz, melyek az ismert fehérje-kódoló gének majdnem 2.5\%-át teszik ki; a rizs esetében pedig ezernél is több RLK kódoló gén ismert (Shiu és mtsai. 2004).

Az Arabidopsis RLK-k körülbelül 25\%-a csupán kináz doménnel rendelkezik extracelluláris jelfelfogó szakasz és transzmembrán regió nélkül, így ezeket összefoglalóan receptor-szerü citoplazmatikus kinázoknak (RLCK) nevezték el. Lúdfüben az RLCK kinázok csoportja 12 osztályba sorolható, melyek összesen mintegy 193 fehérjét számlálnak. 
A növényi RLCK kinázok funkciójával kapcsolatos ismereteink csak néhány fehérjére korlátozottak, de nagyon valószínü, hogy központi szerepet játszanak az RLK kinázok által észlelt külső jelek sejten belüli továbbításában (Zhou és mtsai. 1995; Murase és mtsai. 2004).

Nemrégiben munkacsoportunk egy németországi csoporttal egyidőben beszámolt arról, hogy a növényi ROP GTP-ázok közvetlen kölcsönhatásban állnak egyes receptorszerü citoplazmatikus kinázokkal (RLCK VI. osztály) lúdfü (Molendijk és mtsai. 2008) és lucerna (Dorjgotov et al. 2009) növényekben. Ezenfelül bizonyítékokkal szolgáltunk arra, hogy Medicago truncatula és Arabidopsis thaliana esetében az RLCK VI. osztályba tartozó kinázokat a GTP-kötött ROP GTP-ázok in vitro specifikusan aktiválják, mely tény alátámasztja azon nézetünket, hogy a növényi Rho (ROP) G-proteinek közvetlenül szabályozhatják ezen RLCK kinázok jelátvitelét.

Egy másik, eltérő kinázcsaládba tartozó ún. ciszteinben gazdag citoplazmatikus receptor kinázról (NCRK) szintén kimutatták, hogy kölcsönhatásban állhat a ROP GTPázokkal (Molendijk és mtsai. 2008). Ezeknek a növényekben specifikus ROPkölcsönható kinázoknak egyike sem rendelkezik olyan ismert fehérje doménnel vagy motívummal, mely összfüggésbe hozható lenne a ROP GTPázok kötésének képességével.

A növényspecifikus ROP GTP-ázok a növények növekedése, fejlődése és a környezeti ingerekre adott válaszai során számos folyamatban molekuláris kapcsolóként viselkednek. Feltételezhető, hogy a velük kölcsönható RLCK kinázok is elhelyezhetőek ezekben a ROP-függő jelátviteli útvonalakban.

Annak ellenére, hogy a lúdfü RLCK VI. osztályába tartozó mintegy tizennégy kináz feltételezhetően jelentőséggel bír a ROP GTP-ázok közvetítette jelátvitelben, ennek a kináz családnak a tagjairól alig néhány gyakorlati/funkcionális információ áll rendelkezésre. Ezért elsődleges célunk a lúdfü (Arabidopsis thaliana) RLCK VI. osztályába tartozó fehérje kinázok jellemzése volt.

Az ide tartozó szekvenciák összehasonlítása nyomán fény derült arra, hogy a 14 fehérje két csoportot alkot és a csoportokon belül bizonyos szekvencia párok fokozott hasonlóságot mutatnak. Ez a tény arra utal, hogy a génkettőződés jelentős szerepet játszhatott ennek a családnak a kialakulása során is. Az RLCK VI. családba tartozó fehérjék egymással magasfokú hasonlóságot mutatnak, legföképpen a kináz doménjükön 
belül, de eltérnek a közeli rokonságban álló más kinázcsaládoktól. Megállapítottuk, hogy számos tagjuk az N-terminális részen egy UspA ("universal stress protein”) domént hordoz (B csoport tagjai), vagy pedig egy szerinben gazdag régióval rendelkeznek ugyanitt (A csoport tagjai).

Hogy az RLCK VI. kinázok lehetséges biológiai szerepét feltárjuk, valósidejü kvantitatív PCR segítségével megvizsgáltuk relatív génkifejeződésüket a lúdfü növény különböző szerveiben (gyökér, tőlevélrózsa, virágzati száron levő levelek, virágzati szár, virágbimbó, kinyílt virág, becő, valamint sejtkúltúra), illetve ezenfelül különböző stressz és hormonkezeléseknek kitett csíranövényekben.

Az RLCK VI. család génjei változatos génkifejeződési mintázatot mutattak különböző növényi szervekben, valamint stressz- és hormonkezelések hatására egyaránt. Bizonyos esetekben a paralóg gének hasonló expressziós mintázattal rendelkeztek. Az adatok arra utalnak, hogy az RLCK VI. kináz család számos tagjának aktivitása a növény fejlödése, valamint környezeti stresszválaszok során transzkripcionális szinten szabályzott. A szigorúan szabályzott és általánosságban alacsony kifejeződésük azt jelzi, hogy ezek a fehérjék inkább specifikus mint általános sejtfolyamatokban játszhatnak szerepet.

Korábban már említettük, hogy a lucerna és lúdfü RLCK VI. kináz családját a ROP GTP-ázok specifikusan aktiválják (Dorjgotov és mtsai. 2009). Azon álláspontunk alátámasztása érdekében, miszerint az RLCK VI. kinázok valóban ROP effektorként hatnak a növényekben, megvizsgáltuk a ROP és RLCK VI_A kinázok génkifejeződésének korrelációját in silico microarray adatok alapján. Ezenfelül, hogy teljes képet kaphassunk a ROP-függő jelátviteli útvonalakban szerepet játszó lehetséges partnerekről, minden ismert ROP szabályzót és effektort bevontunk ebbe a vizsgálatba.

A microarray expressziós adatok hierarchikus csoportosítása rámutatott arra, hogy egyértelmű korreláció áll fenn számos vizsgált fehérje szerv/szövetspecifikus expressziója között. Öt nagyobb expressziós csoport (A-E) létrehozására volt lehetőség. Ezen csoportok azokat a géneket tartalmazzák, melyek vagy csak az adott szerv(ek)ben, vagy ott nagyobb mennyiségben fejeződnek ki, úgymint a pollenben és virágban; szárban, nóduszokban és hipokotilban; gyökérben; hajtás- és gyökércsúcsban, virágban és becőben; valamint az egész növényben. 
Annak érdekében, hogy az RLCK kinázok és ROP GTP-ázok specifikus kölcsönhatásait megvizsgáljuk, egy élesztő két hibrid módszeren alapuló fehérje-fehérje kölcsönhatási mátrixot hoztunk létre tíz db. RLCK VI. kináz (5-5 mind az A, mind a B csoportból) és $8 \mathrm{db}$ Arabidopsis ROP GTP-áz bevonásával. A kölcsönhatás csak az RLCK VI. kinázok A csoportjára nézve bizonyult specifikusnak. Mindamellett a B csoportból egy véletlenszerüen kiválasztott kináz szintén kölcsönhatást mutatott egy ROP GTP-ázzal egy in vitro végzett fehérje kölcsönhatási (pull down) kísérlet során. A különböző fehérje-fehérje kölcsönhatási vizsgálatok során kapott eltérő eredményekért feltehetően a más-más körülmények voltak felelősek, melyek stibilizálhatták, vagy gyengíthették a kölcsönhatást. Mindazonáltal az in vitro kináz aktivitás mérések (lásd. később) alapján feltételezzük, hogy az élesztös kísérletben kapott kölcsönhatási mintázatok specifikusabbak és jobban imitálják a növényi sejtekben történő folyamatokat.

A fehérjeszekvencia analízis felfedte, hogy az élesztő két-hibrid vizsgálat során a ROP GTP-ázokkal kölcsönhatást mutató A illetve a kölcsönhatást nem mutató B csoporthoz tartozó kinázok szekvenciáiban számos jellemző különbség mutatkozik. Íly módon sikerült az RLCK-ROP kötődésben feltételezhetően szerepet játszó motívumokat azonosítani.

Annak ellenére, hogy in vitro a ROP GTP-ázok kölcsönhatottak többféle RLCK kinázzal is, a kináz aktivitás mérések egyértelmúen azt jelezték, hogy funkcionális kölcsöhatás csak a ROP fehérjék és az RLCK VI. kinázok A csoportja között van. Vizsgálataink szerint a ROP GTP-ázok nem tudtak aktiválni egy a B csoporthoz tartozó (AtRLCK VI_B3), valamint egy másik, a VII. osztályba tartozó, RLCK kinázt, mely szintén arra utal, hogy specifikusan csak az RLCK VI. kinázcsalád A csoportjával állnak funkcionális kölcsönhatásban. A kináz foszforilációs aktivitásának növelésében a GTPázok GTP-kötött konformációjának jelenléte bizonyult a leghatékonyabbnak. Az a tény, hogy az RLCK VI_A kinázokat specifikusan és erősen a GTP-ázok aktív konformációja aktiválja arra utal, hogy feltehetően ezek a kinázok a ROP GTP-ázok lehetséges effektorai, azaz a ROP GTPázokra érkező jelet továbbítják további célmolekulák felé. Molendijk és munkatársai (2008) a ROP-RLCK kölcsönhatás első leközlői ezt a lehetőséget elvetették. Ök nem tudtak megfigyelni sem ROP GTP-áz függő in vitro 
autofoszforilációt sem in vivo szubsztrát foszforilációt a rovar sejtekből immunoprecipitált ROP-RLCK komplex esetében.

Bár valószínü, hogy az RLCK VI_A kinázok ROP effektorokként müködnek, az nem világos, hogy a GTP-ázok hogyan képesek aktiválni a kinázokat. A kinázok ROPkötésért felelős régiója nem ismert, de valamivel többet tudunk a ROP GTPázok kináz aktiválásáért felelős regiójáról. A RhoGTP-ázok egy specifikus régiójának, az úgynevezett Rho-inszert regiónak bizonyítottan köze van az effektorok kötéséhez és aktivációjához (Karnoub és mtsai. 2004). Az emberi és élesztő Rac, Rho és Cdc42 fehérjékkel összehasonlítva a növényi ROP GTP-ázok egy jellegzetes különbségeket felmutató inszert régióval rendelkeznek, mely arra utal, hogy a növények specifikus ROP GTP-áz effektorokkal rendelkezhetnek (Berken 2008). Laboratóriumunkban bizonyításra került, hogy ez a specifikus regió fontos szerepet játszik az MtRRK1 (MtRLCK VI_A2) kináz ROP GTPáz általi aktiválása során (Dorgjotov és mtsai. 2009).

A funkcionális ROP-RLCK kölcsönhatás biológiai szerepének meghatározása még elöttünk áll. Ennek érdekében a RLCK VI_A2 gént túltermelö, illetve erre a génre nézve csendesített transzgénikus növények létrehozásába kezdtünk.

A sejtek polarizációja szorosan kapcsolódik a növények fejlődéséhez, növekedéséhez. Az jól ismert tény, hogy a növényi sejtek mind a növényspecifikus, mind a konzervált polaritási információkat olyan ősi mechanizmusok segítségével integrálják, mely folyamatokban a Rho GTP-áz családok is részt vesznek. Ezenfelül a sejtváz dinamikájának/újraszerveződésének irányítása, valamint a polaritás kialakulásához és fenntartásához szükséges vezikuláris folyamatok szintén Rho GTP-áz szabályozott folyamatok

Azt gyanítjuk, hogy az AtRLCK VI_A2 fehérje, mint potenciális ROP effektor kináz, szerepet játszhat a polaritás kialakulásában is. Az irányított sejtmegnyúlás (pl. levélszőrök, trichómák) és csúcsi növekedés (pl. pollencső) két alapvető folyamat, melyek nélkülözhetetlenek a növények poláris sejttípusainak morfogeneziséhez.

További bizonyításra váró előzetes kísérleteink alapján mind az AtRLCK VI_A2 gén túltermeltetése, mind a csendesítése megzavarja a pollen poláris növekedését, valamint hatással lehet a csillagszőrők elágazódására. Az AtRLCK VI_A2 túltermeltetése megnöveli a kevesebb elágazódással rendelkező csillagszőrök gyakoriságát, míg a gén 
csendesítése a trichómákon több elágazódás megjelenéséhez vezet, igaz kis gyakorisággal. Az AtRLCK VI_A2 túltermeltetése pollencsőben a cső végének kettéágazódásához vezethet. Ugyanezen gén elcsendesítése a pollencső végének buborékszerü kiszélesedését okozhatja. További részletes vizsgálatokat végzünk majd, hogy alátámasszuk az RLCK VI_A kinázok feltételezett szerepét az említett, valamint további ROP GTP-ázok által szabályozott folyamatokban (pl. kórokozó fertőzésre adott válaszok, reaktív oxigén fajták termelése), ezáltal igazolni kívánjuk, hogy az RLCK kinázok valóban növényspecifikus ROP GTP-áz effektor kinázok.

Összefoglalásképpen elmondhatjuk, hogy az Arabidopsis RLCK VI_A kinázok in vitro kölcsönhatnak a ROP GTP-ázokkal, valamint in vitro aktiválódnak általuk. Ezenfelül génkifejeződési mintázatuk vizsgálata és a transzgénikus növények jellemzése azt engedi feltételeznünk, hogy ezek a kinázok ROP GTP-áz effektorokként müködhetnek a növényekben. Ezen jeltovábbító lépésnek számos növényspecifikus vonatkozása van. Ezért további vizsgálataink érdekes betekintést nyújthatnak majd a sejtszintü jelátviteli mechanizmusok evolúciójába is és értékes információkkal gazdagíthatnak bennünket a növények egyedfejlődésének és stresszválaszának szabályozásával kapcsolatban. 


\section{References}

- Acharya, B., Raina, S., Maqbool, S., Jagadeeswaran, G., Mosher, S., Appel, H., Schultz, J., Klessig, D., Raina, R., (2007) Overexpression of CRK13, an Arabidopsis cysteine-rich receptor-like kinase, results in enhanced resistance to Pseudomonas syringae. Plant Journal. 50, 488-499

- Adams, K., Cronn, R., Percifield, R., Wendel, J., (2003) Genes duplicated by polyploidy show unequal contributions to the transcriptome and organ-specific reciprocal silencing. Proceedings of the National Academy of Sciences USA. 100, 4649-4654

- Agatep, R., Kirkpatrick, R., Parchaliuk, D., Woods, R., Gietz, R., (1998) Transformation of Saccharomyces cerevisiae by the lithium acetate/single-stranded carrier DNA/polyethylene glycol (LiAc/ss-DNA/PEG) protocol. Technical Tips Online (BPhttp://tto.trends.comBP)

- Agrawal, G., Iwahashi, H., Rakwal, R., (2003) Small GTPase 'Rop': molecular switch for plant defense responses. FEBS Letters. 546, 173-180

- Albrecht, C., Russinova, E., Hecht, V., Baaijens, E., de Vries, S., (2005) The Arabidopsis thaliana Somatic Embryogenesis Receptor-Like Kinases1 and 2 control male sporogenesis. Plant Cell. 17, 3337-3349

- Aldrich, J., Cullis, C., (1993) RAPD analysis in flax: optimization of yield and reproducibility using KlenTaq1 DNA polymerase, Chelex 100, and gel purification of genomic DNA. Plant Molecular Biology Reporter. 11, 128-141

- Allan, A., Fluhr, R., (1997) Two distinct sources of elicited reactive oxygen species in tobacco epidermal cells. Plant Cell. 9, 1559-1572

- Allwood, E., Anthony, R., Smertenko, A., Reichelt, S., Drobak, B., Doonan, J., Weeds, A., Hussey, P., (2002) Regulation of the Pollen-Specific Actin-Depolymerizing Factor LlADF1. Plant Cell. 14, 2915-2927

- An, Y., Huang, S., McDowell, J., McKinney, E., Meagher, R., (1996) The Arabidopsis ACT1/ACT3 actin subclass is expressed in organ primordial and mature pollen. Plant Cell. $8,15-30$

- Anthony, R., Khan, S., Costa, J., Pais, M., Bögre, L., (2006) The Arabidopsis protein kinase PTI1-2 is activated by convergent phosphatidic acid and oxidative stress signaling pathways downstream of PDK1 and OXI1. Journal of Biological Chemistry. 281, 3753637546

- Apel, K., Hirt, H., (2004) Reactive oxygen species: Metabolism,oxidative stress, and signal transduction. Annual Review of Plant Biology. 55, 373-399

- Bagrodia, S., Cerione, R., (1999) Pak to the future. Trends in Cell Biology. 9, 350-355

- Basu, D., El-Assal, S., Le, J., Mallery, E., Szymanski, D., (2004) Interchangeable functions of Arabidopsis PIROGI and the human WAVE complex subunit SRA1 during leaf epidermal development. Development. 131, 4345-55

- Basu, D., Le, J., Zakharova, T., Mallery, E., Szymanski, D., (2008) A SPIKE1 signaling complex controls actin-dependent cell morphogenesis through the heteromeric WAVE and ARP2/3 complexes. Proceedings of the National Academy of Sciences USA. 105, 4044-9

- Baxter-Burrell, A., Yang, Z., Springer, P., Bailey-Serres, J., (2002) RopGAP4-dependent Rop GTPase rheostat control of Arabidopsis oxygen deprivation tolerance. Science. 296, 20262028

- Bent, A., Mackey, D., (2007) Elicitors, effectors, and R genes: the new paradigm and a lifetime supply of questions. Annual Review of Phytopathology. 45,399-436

- Berken, A. (2006) ROPs in the spotlight of plant signal transduction. Cellular and Molecular Life Sciences. 63, 2446-59. 
- Berken, A. and Wittinghofer, A., (2008) Structure and function of Rho-type molecular switches in plants. Plant Physiology and Biochemistry. 46, 380-93

- Berken, A., Thomas, C., Wittinghofer, A., (2005) A new family of RhoGEFs activates the Rop molecular switch in plants. Nature. 436, 1176-80

- Bischoff, F., Vahlkamp, L., Molendijk, A., Palme, K., (2000) Localization of AtRop4 and AtRop6 and interaction with the guanine nucleotide dissociation inhibitor AtRhoGDI1 from Arabidopsis. Plant Molecular Biology. 42, 515-30

- Blanc, G., Wolfe, K., (2004) Functional divergence of duplicated genes formed by polyploidy during Arabidopsis evolution. Plant Cell. 16, 1679-1691

- Bloch, D., Hazak, O.,Lavy, M., Yalovsky, S., (2008) A novel ROP/RAC GTPase effector integrates plant cell form and pattern formation. Plant Signaling \& Behaviour. 3(1), 41-43

- Bloch, D., Lavy, M., Efrat, Y., Efroni, I., Bracha-Drori, K., Abu-Abied, M., Sadot, E., Yalovsky, S., (2005) Ectopic expression of an activated RAC in Arabidopsis disrupts membrane cycling. Molecular Biology of the Cell. 16, 1913-1927

- Boavida, L., McCormick, S., (2007) Temperature as a determinant factor for increased and reproducible in vitro pollen germination in Arabidopsis thaliana. Plant Journal. 52(3),57082

- Bokoch, G., (2000) Regulation of cell function by Rho family GTPases. Immunology Research. 21, 139-48

- Bokoch, G., (2003) Biology of the p21-activated kinases. Annual Review of Biochemistry. 72, 743-81.

- Boureux, A., Vignal, E., Faure, S., Fort, P., (2007) Evolution of the Rho family of ras-like GTPases in eucaryotes. Molecular Biolology and Evolution. 24, 203-216

- Bourne, H., Sanders, D., McCormick, F., (1991) The GTPase superfamily: conserved structure and molecular mechanism. Nature. 349,117-127

- Brembu, T., Winge, P., Bones, A., (2005) The small GTPase AtRAC2/ROP7 is specifically expressed during late stages of xylem differentiation in Arabidopsis. Journal of Experimental Botany. 56, 2465-2476

- Brembu, T., Winge, P., Bones, A., Yang, Z., (2006) A RHOse by any other name: a comparative analysis of animal and plant Rho GTPases. Cell Research. 16, 435-45

- Brennwald, P., Rossi, G., (2007) Spatial Regulation of Exocytosis and Cell Polarity: Yeast as a Model for Animal Cells. FEBS Letters. 581, 2119-24

- Burbelo, P., Drechsel, D., Hall, A., (1995) A conserved binding motif defines numerous candidate target proteins for both $\mathrm{Cdc} 42$ and Rac GTPases. Journal of Biological Chemistry. 270, 29071-29074

- Bustelo, X., Sauzeau, V., Berenjeno, I., (2007) GTP-binding proteins of the Rho/Rac family: regulation, effectors and functions in vivo. Bioessays. 29, 356-70

- Butenko, M., Vie, A., Brembu, T., Aalen, R., Bones, A., (2009) Plant peptides in signalling: looking for new partners. Trends in Plant Science. 14, 255-263

- Carol, J., Takeda, S., Linstead, P., Durrant, M., Kakesova, H., Derbyshire, P., Drea, S., Zarsky, V., and Dolan, L., (2005) A RhoGDP dissociation inhibitor spatially regulates growth in root hair cells. Nature. 438, 1013-1016

- Carol, R., Takeda, S., Linstead, P., (2005) A RhoGDP dissociation inhibitor spatially regulates growth in root hair cells. Nature. 438, 1013-6

- Casneuf, T., De Bodt, S., Raes, J., Maere. S., Van de Peer. Y., (2006) Nonrandom divergence of gene expression following gene and genome duplications in the flowering plant Arabidopsis thaliana. Genome Biology. 7, R13

- Chan, J., Pauls, P., (2007) Brassica napus Rop GTPases and their expression in microspore cultures. Planta. 225, 469-84 
- Charrier, B., Champion, A., Henry, Y., Kreis, M., (2002) Expression profiling of the whole Arabidopsis shaggy-like kinase multigene family by real-time reverse transcriptasepolymerase chain reaction. Plant Physiology. 130, 577-590

- Chen, C., Cheung, A., Wu, H., (2003) Actin-depolymerizing factor mediates Rac/Rop GTPase-regulated pollen tube growth. Plant Cell.15, 237-49

- Cheung, A., Chen, C., Tao, L., Andreyeva, T., Twell, D., Wu, H., (2003) Regulation of pollen tube growth by Rac-like GTPase. Journal of Experimental Botany. 54, 73-81

- Cheung, A., Wu, H., (2004) Overexpression of an Arabidopsis formin stimulates supernumerary actin cable formation from pollen tube cell membrane. Plant Cell. 16(1), 257-69.

- Chevalier, D., Batoux, M., Fulton, L., Pfister, K., Yadav, R., Schellenberg, M., Schneitz K., (2005) STRUBBELIG defines a receptor kinase-mediated signaling pathway regulating organ development in Arabidopsis. Proceedings of the National Academy of Sciences USA. 102, 9074-9079

- Chinchilla, D., Zipfel, C., Robatzek, S., Kemmerling, B., Nürnberger, T., Jones, J., Felix, G., Boller, T., (2006) A flagellin-induced complex of the receptor FLS2 and BAK1 initiates plant defence. Nature. 448, 497-500

- Chomczynski, P., Sacchi, N., (1987) Single-step method of RNA isolation by acid guanidinium thiocyanate phenol chloroform extraction. Analitical Biochemistry. 162,156159

- Christensen, T., Vejlupkova, Z., Sharma, Y., Arthur, K., Spatafora, J., Albright, C., Meeley, R., Duvick, J., Quatrano, R., Fowler, J., (2003) Conserved subgroups and developmental regulation in the monocot rop gene family. Plant Physiology. 133, 1791-1808.

- Clark, S., Williams, R., Meyerowitz, E., (1997) The CLAVATA1 gene encodes a putative receptor kinase that controls shoot and floral meristem size in Arabidopsis. Cell. 89, 575585

- Colcombet, J., Boisson-Dernier, A., Ros-Palau, R., Vera, C., Schroeder, J., (2005) Arabidopsis Somatic Embryogenesis Receptor Kinases1 and 2 are essential for tapetum development and microspore maturation. Plant Cell. 17, 3350-3361

- Cole, R., Fowler, J., (2006) Polarized growth: maintaining focus on the tip. Current Opinion in Plant Biology. 9, 579-88

- Colicelli, J., (2004) Human RAS superfamily proteins and related GTPases. Science STKE. 250 RE13

- Côté, J., Vuori, K., (2007) GEF what? Dock180 and related proteins help Rac to polarize cells in new ways. Trends in Cell Biology. 17, 383-93

- Cotteret, S., Chernoff, J. (2002) The evolutionary history of effectors downstream of Cdc42 and Rac. Genome Biology. 3, reviews0002.1-reviews0002.8

- Cvrcková, F., (2000) Are plant formins integral membrane proteins?.Genome Biology 1(1)

- Czechowski, T., Stitt, M., Altmann, T., Udvardi, M., Scheible, W., (2005) Genome-wide identification and testing of superior reference genes for transcript normalization in Arabidopsis. Plant Physiology. 139, 5-17

- Dan, I., Watanabe, N., Kusumi, A., (2001) The Ste20 group kinases as regulators of map kinase cascades. Trends in Cell Biology. 11, 220-30

- Deeks, M., Hussey, P., (2005) Arp2/3 and SCAR: plants move to the fore. Nature Reviews Molecular Cell Biology. 6, 954-964.

- Deeks, M., Hussey, P., Davies, B., (2002) Formins: intermediates in signal-transduction cascades that affect cytoskeletal reorganization. Trends in Plant Science. 7(11), 492-8 
- Delmer, D., Pear, J., Andrawis, A., Stalker, D., (1995) Genes encoding small GTP-binding proteins analogous to mammalian Rac are preferentially expressed in developing cotton fibers. Molecular Genetics and Genomics. 248, 43- 51

- DerMardirossian, C., Bokoch, G., (2005) GDIs: central regulatory molecules in Rho GTPase activation. Trends in Cell Biology. 15, 356-363

- DeYoung, B., Bickle, K., Schrage, K., Muskett, P., Patel, K., Clark, S., (2006) The CLAVATA1-related BAM1, BAM2 and BAM3 receptor kinase-like proteins are required for meristem function in Arabidopsis. Plant Journal. 45, 1-16

- Diener, A., Ausubel, F., (2005) Resistance to Fusarium oxysporum 1, a dominant Arabidopsis disease-resistance gene, is not race specific. Genetics. 171, 305-321

- Dorjgotov, D., Jurca, M., Dunainé-Fodor, C., Szücs, A.,Ötvös, K., Klement, E., Bíró, J., Fehér, A. (2009) Plant Rho-type (Rop) GTPase-dependent activation of receptor-like cytoplasmic kinases in vitro. FEBS Letters. 583(7), 1175-82

- Dransart, E., Olofsson, B., Cherfils, J., (2005) RhoGDIs revisited: novel roles in Rho regulation. Traffic. 6, 957-966

- Duarte, J., Cui, L., Wall, P., Zhang, Q., Zhang, X., Leebens-Mack, J., Ma, H., Altman, N., dePamphilis, C., (2006) Expression pattern shifts following duplication indicative of subfunctionalization and neofunctionalization in regulatory genes of Arabidopsis. Molecular Biology and Evolution. 23, 469-478

- Dunn, K., Espino, P., Drobic, B., He, S., Davie, J., (2005) The Ras-MAPK signal transduction pathway, cancer and chromatin remodeling. Biochemistry and Cell Biology. 83, $1-14$

- Endre, G., Kereszt, A., Kevei, Z., Mihacea, S., Kalo, P., Kiss, G., (2002) A receptor kinase gene regulating symbiotic nodule development. Nature. 417,962-966

- Escobar-Restrepo, J., Huck, N., Kessler, S., Gagliardini, V., Gheyselinck, J., Yang, W., Grossniklaus, U., (2007) The FERONIA receptor-like kinase mediates male-female interactions during pollen tube reception. Science. 317, 656-660

- Ettwiller, L., Veitia, R., (2007) Protein coevolution and isoexpression in yeast macromolecular complexes. Comparative and Functional Genomics. 58721.

- Eyuboglu, B., Pfister, K., Haberer, G., Chevalier, D., Fuchs, A., Mayer, K., Schneitz, K., (2007) Molecular characterisation of the STRUBBELIG RECEPTOR FAMILY of genes encoding putative leucine-rich repeat receptor-like kinases in Arabidopsis thaliana. BMC Plant Biology. 7, 16

- Fehér, A., Jurca, M., Fodor-Dunaine C., Dorjgotov D., (2008) Regulation of ROP GTPase Signalling at the Gene Expression Level: A Review. The Open Plant Science Journal. 2, $37-46$

- Fields, S, Song, O., (1989) A novel genetic system to detect protein-protein interactions. Nature. 340, 245-6

- Florentino, L., Santos, A., Fontenelle, M., Pinheiro, G., Zerbini, F., Baracat-Pereira, M., Fontes, E. (2006) A PERK-like receptor kinase interacts with the geminivirus nuclear shuttle protein and potentiates viral infection. The Journal of Virology. 80, 6648-6656

- Folkers, U., Berger, J., Huelskamp, M., (1997) Cell morphogenesis of trichomes in Arabidopsis: differential control of primary and secondary branching by branch initiation regulators and cell growth. Development. 124, 3779-3786

- Fontes, E., Santos, A., Luz, D., Waclawovsky, A., Chory, J., (2004) The geminivirus nuclear shuttle protein is a virulence factor that suppresses transmembrane receptor kinase activity. Genes \& Development. 18, 2545-2556

- Foreman, J., Demidchik, V., Bothwell, J., Mylona, P., Miedema, H., Torres, M., Linstead, P., Costa, S., Brownlee, C., Jones, J., Davies, J., and Dolan, L., (2003) Reactive oxygen species produced by NADPH oxidase regulate plant cell growth. Nature. 422, 442-446 
- France, Y., Boyd, C., Coleman, J., Novick, P., (2006) The polarity-establishment component Bem1p interacts with the exocyst complex through the Sec15p subunit. Journal of Cell Science. 119, 876-888

- Fraser, H., Hirsh, A., Wall, D., Eisen, M., (2004) Coevolution of gene expression among interacting proteins. Proceedings of the National Academy of Sciences USA. 101, 9033-8

- Freeman, J., Abo, A., Lembeth, J., (1996) Rac “insert region"' is a novel effector region that is implicated in the activation of NADPH oxidase, but not PAK65. Journal of Biological Chemistry. 271, 19794-19801

- Fu, Y., Gu, Y., Zheng, Z., Wasteneys, G., Yang, Z., (2005) Arabidopsis interdigitating cell growth requires two antagonistic pathways with opposing action on cell morphogenesis. Cell. 120, 687-700

- Fu, Y., Wu, G., Yang, Z., (2001) Rop GTPase-dependent dynamics of tip-localized F-actin controls tip growth in pollen tubes. Journal of Cell Biology. 152, 1019-1032

- Fujiwara, M., Hamada, S., Hiratsuka, M., Fukao, Y., Kawasaki, T., Shimamoto, K., (2009) Proteome analysis of detergent-resistant membranes (DRMs) associated with OsRac1mediated innate immunity in rice. Plant Cell Physiology. 50(7), 1191-200.

- Fulop, K., Tarayre, S., Kelemen, Z., Horvath, G., Kevei, Z., Nikovics, K., Bako, L., Brown, S., Kondorosi, A., Kondorosi, E., (2005) Arabidopsis anaphase-promoting complexes: multiple activators and the wide range of substrates might keep APC perpetually busy. Cell Cycle. 4(8), 1084-1092

- Gapper, C., Dolan, L., (2006) Control of plant development by reactive oxygen species. Plant Physiology. 141, 341-345

- Geyer, M, Wittinghofer, A., (1997) GEFs, GAPs, GDIs and effectors: taking a closer (3D) look at the regulation of Ras-related GTP-binding proteins. Current Opinion in Structural Biology. 7, 786-92

- Gomez-Gomez, L., Boller, T., (2000) FLS2: an LRR receptor-like kinase involved in the perception of bacterial elicitor flagellin in Arabidopsis. Molecular Cell. 5, 1003-1011

- Grefen, C., Harter, K., (2004) Plant two-component systems: principles, functions, complexity and cross talk. Planta. 219, 733-742

- Grennan, A, (2006) Genevestigator. facilitating web-based gene-expression analysis. Plant Physiology.141, 1164-6

- Groom, Q., Torres, M., et al. (1996) rbohA, a rice homologue of the mammalian gp91phox respiratory burst oxidase gene. The Plant Journal. 10, 515-522.

- Gu, Y., Fu, Y., Dowd, P., et al., (2005) A Rho family GTPase controls actin dynamics and tip growth via two counteracting downstream pathways in pollen tubes. The Journal of Cell Biology. 169, 127-38

- Gu, Y., Li, S., Lord, E., Yang, Z., (2006) Members of a novel class of Arabidopsis Rho guanine nucleotide exchange factors control Rho GTPase-dependent polar growth. Plant Cell. 18, 366-81

- Gu, Y., Vernoud, V., Fu, Y., Yang, Z., (2003) ROP GTPase regulation of pollen tube growth through the dynamics of tip-localized F-actin. Journal of Experimental Botany. 54, 93-101

- Gu, Y., Wang, Z., Yang, Z., (2004) ROP/RAC GTPase an old new master regulator for plant signaling. Current Opinion in Plant Biology. 7, 527-536

- Haberer, G., Hindemitt, T., Meyers, B., Mayer, K., (2004) Transcriptional similarities, dissimilarities, and conservation of ciselements in duplicated genes of Arabidopsis. Plant Physiology. 136, 3009-3022

- Haffani, Y., Silva, N., Goring, D., (2004) Receptor kinase signaling in plants. Canadian Journal of Botany. 82, 1-15 
- Hagen, G., Guilfoyle, T., (2002) Auxin-responsive gene expression: genes, promoters and regulatory factors. Plant Molecular Biology. 49(3-4),373-85

- Halliwell, B., Gutteridge, J., (1989) Free radicals in Biology and Medicine. 2nd Edition of Clarendon Press, Oxford

- Hancock, J., Magee, A., Childs, J., Marshall, C., (1989) All Ras proteins are polyisoprenylated but only some are palmitoylated. Cell. 57(7), 1167-77

- Hassanain, H., Sharma, Y., $\quad$ Moldovan, L., Khramtsov, V., $\quad$ Berliner, L., $\quad$ Duvick, J., Goldschmidt-Clermont, P., (2000) Plant rac proteins induce superoxide production in mammalian cells. Biochemical and Biophysical Research Communications. 272,783-788

- Hazak, O., Yalovsky, S., (2010) An auxin regulated positive feedback loop integrates Rho modulated cell polarity with pattern formation. Plant Signaling and Behavior. 5, 709-711

- Hellmann, H., Estelle, M., (2002) Plant development: regulation by protein degradation. Science. 297(5582),793-7

- Henzler, T., Steudle, E., (2000) Transport and metabolic degradation of hydrogen peroxide in Chara corallina: Model calculations and measurements with the pressure probe suggest transport of $\mathrm{H}_{2} \mathrm{O}_{2}$ across water channels. Journal of Experimental Botany. 51, 2053-2066

- Hepler, P., Vidali, L., Cheung, A., (2001) Polarized cell growth in higher plants. Annual Review of Cell and Developmental Biology. 17, 159-187

- Herrmann, M., Pinto, S., Kluth, J., Wienand, U., Lorbiecke, R., (2006) The PTI1-like kinase ZMPTI1a from maize (Zea mays L.) colocalizes with callose at the plasma membrane of pollen and facilitates a competitive advantage to the male gametophyte. BMC Plant Biology. 6, 22

- Hofmann, C., Shepelev, M., Chernoff, J. (2004) The genetics of PAK. Journal of Cell Science.117, 4343-54

- Hong, Z., Zhang, Z., Olson, J., Verma, D., (2001) A novel UDP-glucose transferase is part of the callose synthase complex and interacts with phragmoplastin at the forming cell plate. Plant Cell.13, 769-7

- Hou, X., Tong, H., Selby, J., Dewitt, J., Peng, X., He, Z., (2005) Involvement of a cell wallassociated kinase, WAKL4, in Arabidopsis mineral responses. Plant Physiology. 139, $1704-1716$

- Huang, S., An, Y., McDowell, J., McKinney, E., Meagher, R., (1997) The Arabidopsis ACT11 actin gene is strongly expressed in tissues of the emerging inflorescence, pollen, and developing ovules. Plant Molecular Biology. 33, 125-139

- Hülskamp, M., Misera, S., Jürgens, G., (1994) Genetic dissection of trichome cell development in Arabidopsi. Cell. 76, 555-566

- Hwang, J., Gu, Y., Lee, Y., (2005) Oscillatory ROP GTPase activation leads the oscillatory polarized growth of pollen tubes. Molecular Biology of the Cell. 16, 5385-5399

- Hwang, J., Wu, G., Yan, A., Lee, Y., Grierson C., Yang, Z., (2009) Pollen-tube tip growth requires a balance of lateral propagation and global inhibition of Rho-family GTPase activity. Journal of Cell Science. 123, 340-350

- Ichinose, Y., Toyoda, K., Barz, W., (1999) cDNA cloning and gene expression of three small GTP-binding proteins in defense response of chickpea. Biochimica et Biophysica Acta. 489(2-3), 462-6

- Ishida, T., Kurata, T., Okada, K., Wada, T., (2008) A Genetic Regulatory Network in the Development of Trichomes and Root Hairs. Annual Review of Plant Biology. 59, 365-386

- Jerome, J., Kristof, D., Aurine, V., Dirk, I., Lieven, D, (2004) Conditional, recombinasemediated expression of genes in plant cell cultures. The Plant Journal. 37, 889-896 
- Johnston, C., Temple, B., et al., (2007) Comment on "A G protein coupled receptor is a plasma membrane receptor for the plant hormone abscisic acid. Science. 318(5852), 914

- Jones, A., (2002) G-protein-coupled signaling in Arabidopsis. Current Opinion in Plant Biology. 5(5), 402-7

- Jones, M., Shen, J., Fu, Y., Li, H., Yang, Z., Grierson, C., (2002) The Arabidopsis Rop2 GTPase is a positive regulator of both root hair initiation and tip growth. Plant Cell. 14, 763-76

- Jones, A., Assmann, A., (2004) Plants: the latest model system for G-protein research. EMBO Reports. 5(6), 572-8

- Jones, M., Raymond, M., Yang, Z., and Smirnoff, N., (2007) NADPH oxidase-dependent reactive oxygen species formation required for root hair growth depends on ROP GTPase. Journal of Experimental Botany. 58, 1261-1270

- Joubes, J., De Schutter, K., Verkest, A., Inze, D., De Veylder, L., (2004) Conditional, recombinase-mediated expression of genes in plant cell culture. Plant Journal. 37, 889-896

- Jung, Y., Agrawal, G., Rakwal, R., Kim, J., Lee, M., Choi, P., et al., (2006) Functional characterization of OsRacB GTPase--a potentially negative regulator of basal disease resistance in rice. Plant Physiology and Biochemistry. 44, 68-77

- Jurca, M., Bottka, S., Feher A., (2008) Characterization of a family of Arabidopsis receptorlike cytoplasmic kinases (RLCK class VI). Plant Cell Reports. 27, 739-748

- Kaothien, P., Ok, S., Shuai, P., Wengier, D., Cotter, R., Kelley, D., Kiriakopolos, S., Muschietti, J., McCormick, S., (2005) Kinase partner protein interacts with the LePRK1 and LePRK2 receptor kinases and plays a role in polarized pollen tube growth. Plant Journal. 42, 492-503

- Karnoub, A., Symons, M., Campbell, S., Der, C., (2004) Molecular basis for Rho GTPase signaling specificity. Breast Cancer Research and Treatment. 84, 61-71

- Karnoub, A., Worthylake, D., Rossman,M., Pruitt, W., Campbell, S., Sondek, J., Der, C., (2001) Molecular basis for Rac1 recognition by guanine nucleotide exchange factors. Nature Structural Biology. 8,1037-1041

- Kawasaki, T., Koita, H., Nakatsubo, T., et al., (2006) Cinnamoyl-CoA reductase, a key enzyme in lignin biosynthesis, is an effector of small GTPase Rac in defense signaling in rice. Proceedings of the National Academy of Sciences USA. 103, 230-5

- Kaziro, Y., Itoh, H., Kozasa, T., Nakafuku, M., Satoh, T., (1991) Structure and function of signal-transducing GTP-binding proteins. Annual Review of Biochemistry. 60, 349-400

- Keller, T., Damude, H., et al., (1998) A plant homolog of the neutrophil NADPH oxidase gp91phox subunit gene encodes a plasma membrane protein with $\mathrm{Ca} 2+$ binding motifs. Plant Cell. 10, 255-266.

- Kerk, D., Bulgrien, J., Smith, D., Gribskov, M., (2003) Arabidopsis proteins containing similarity to the universal stress protein domain of bacteria. Plant Physiology.131, 12091219

- Kieffer, F., Elmayan, T., Rubier, S., Simon-Plas, F., Dagher, M., Blein, J., (2000) Cloning of Rac and Rho-GDI from tobacco using an heterologous two-hybrid screen. Biochimie. 82, 1099-105

- Kim, H., Triplett, B., (2004) Characterization of GhRac1 GTPase expressed in developing cotton (Gossypium hirsutum L.) fibers. Biochimica et Biophysica Acta. 1679, 214-21

- Kim, S., Yu, Y., Snesrud, E., Moy, L., Linford, L., Haas, B., Nierman, W., Quackenbush, J., (2005) Transcriptional divergence of the duplicated oxidative stress-responsive genes in the Arabidopsis genome. Plant Journal. 41, 212-220

- Klahre, U., Becker, C., Schmit, A., Kost, B., (2006) Nt-RhoGDI2 regulates Rac/Rop signaling and polar cell growth in tobacco pollen tubes. Plant Journal. 46, 1018-31 
- Klahre, U., Kost, B., (2006) Tobacco RhoGTPase ACTIVATING PROTEIN1 spatially restricts signaling of RAC/ROP to the apex of pollen tubes. Plant Cell. 18, 3033-46

- Ko, J., Beers, E., Han, K., (2006) Global comparative transcriptome analysis identifies gene network regulating secondary xylem development in Arabidopsis thaliana. Molecular Genetics \& Genomics. 276, 517-531

- Kodama, Y., Tamura, T., Hirasawa, W., Nakamura, K., Sano, H., (2009) A novel protein phosphorylation pathway involved in osmotic-stress response in tobacco plants. Biochimie. 91(4), 533-9

- Kost, B., (2008) Spatial control of Rho (Rac-Rop) signaling in tip-growing plant cells. Trends in Cell Biology.18,119-27

- Kost, B., Lemichez, E., Spielhofer, P., (1999) Rac homologues and compartmentalized phosphatidylinositol 4, 5-bisphosphate act in a common pathway to regulate polar pollen tube growth. The Journal of Cell Biology. 145, 317-330

- Kovtun, Y., Chiu, W., Tena, G., Sheen, J., (2000) Functional analysis of oxidative stressactivated mitogen-activated protein kinase cascade in plants. Proceedings of the National Academy of Sciences USA. 97, 2940-2945

- Kunze, G., Zipfel, C., Robatzek, S., Niehaus, K., Boller, T., Felix, G., (2004) The N terminus of bacterial elongation factor Tu elicits innate immunity in Arabidopsis plants. Plant Cell. 16, 3496-3507.

- Kwak, J., Mori, I., Pei, Z., Leonhardt, N., Torres, M., Dangl, J., Bloom, R., Bodde, S., Jones, J., Schroeder, J., (2003) NADPH oxidase AtrbohD and AtrbohF genes function in ROS dependent ABA signaling in Arabidopsis. EMBO Journal. 22, 2623-2633

- Lalonde, S., Ehrhardt, D., Loqué, D., Chen, J., Rhee, S., Frommer, W., (2008) Molecular and cellular approaches for the detection of protein-protein interactions: latest techniques and current limitations. Plant Journal. 53(4), 610-35

- Lavy, M., Bloch, D., Hazak, O., et al., (2007) A novel Rop/Rac effector links cell polarity, root-meristem maintenance, and vesicle trafficking. Current Biology. 17, 947-52

- Lavy, M., Bloch, D., Hazak, O., Gutman, I., Poraty, L., Sorek, N., Sternberg, H., Yalovsky, S., (2007) A Novel ROP/RAC effector links cell polarity, root-meristem maintenance, and vesicle trafficking. Current Biology. 17, 947-952

- Lavy, M., Bracha-Drori, K., Sternberg, H., Yalovsky, S., (2002) A cell-specific, prenylationindependent mechanism regulates targeting of type II RACs. Plant Cell. 14, 2431-2450

- Lavy, M., Yalovsky, S., (2006) Association of Arabidopsis type-II ROPs with the plasma membrane requires a conserved C-terminal sequence motif and a proximal polybasic domain. Plant Journal. 46, 934-947

- Lee, Y., Szumlanski, A., Nielsen, E., Yang, Z., (2008) Rho-GTPase-dependent Factin dynamics coordinate vesicle targeting and exocytosis during tip growth. Journal of Cell Biology. 181, 1155-1168

- Leipe, D., Wolf, Y., Koonin, E., Aravind, L., (2002) Classification and evolution of P-loop GTPases and related ATPases. Journal of Molecular Biology. 317, 41-72

- Lemichez, E., Wu, Y., Sanchez, J., Mettouchi, A., Mathur, J., Chua, N., (2001) Inactivation of AtRac1 by abscisic acid is essential for stomatal closure. Genes \& Development. 15,1808-1816

- Leveleki, L., Mahlert, M., et al., (2004) The PAK family kinase Cla4 is required for budding and morphogenesis in Ustilago maydis. Molecular Microbiology. 54(2), 396-406

- Li, H., Lin, Y., Heath, R., (1999) Control of pollen tube tip growth by a Rop GTPasedependent pathway that leads to tip-localized calcium influx. Plant Cell. 11, 1731-1742

- Li, H., Wu, G., Ware, D., Davis, K, Yang, Z., (1998) Arabidopsis Rho-related GTPases: differential gene expression in pollen and polar localization in fission yeast. Plant Physiology. 118, 407-417 
- Li, J., Chory, J., (1997) A putative leucine-rich repeat receptor kinase involved in brassinosteroid signal transduction. Cell. 90, 929-938

- Li, J., Wen, J., Lease, K., Doke, J., Tax, F., Walker, J., (2002) BAK1, an Arabidopsis LRR receptor-like protein kinase, interacts with BRI1 and modulates brassinosteroid signaling. Cell. 110, 213-222

- Lieberherr, D., Thao, N., Nakashima, A., Umemura, K., Kawasaki, T., Shimamoto, K., (2005) A sphingolipid elicitor-inducible mitogen-activated protein kinase is regulated by the small GTPase OsRac1 and heterotrimeric G-protein in rice 1. Plant Physiology. 138(3), 1644-52

- Lin, H., Wang, X., Ng-Eaton, E., Weinberg, R., Lodish, H., (1992) Expression cloning of the TGF-P type I1 receptor, a functional transmembrane serine/threonine kinase. Cell. 68, 775785

- Lin, Y., Yang, Z., (1997) Inhibition of pollen tube extension by microinjected anti-Rop1Ps antibodies suggest a role for rho-type GTPases in the control of tip growth. Plant Cell. 9, 1647-1659

- Lu, D., Wu, S., Gao, X., Zhang, Y., Shan, L., He, P., (2010) A receptor-like cytoplasmic kinase, BIK1, associates with a flagellin receptor complex to initiate plant innate immunity. Proceedings of the National Academy of Sciences USA. 107, 496-501

- Lundquist, E., (2006) Small GTPases. WormBook.17, 1-18

- Luo, D., Oppenheimer, D., (1999) Genetic control of trichome branch number in Arabidopsis: the roles of the FURCA loci. Development. 126, 5547-57

- Mahlert, M., Leveleki, L., et al., (2006) Rac1 and Cdc42 regulate hyphal growth and cytokinesis in the dimorphic fungus Ustilago maydis. Molecular Microbiology. 59(2): 56778

- Manser, E., Leung, T., Salihuddin, H., Zhao, Z., Lim, L., (1994) A brain serine/threonine protein kinase activated by Cdc42 and Rac1. Nature. 367, 40-46

- Mathur, J., Chua, N., (2000) Microtubule stabilization leads to growth reorientation in Arabidopsis trichomes. Plant Cell. 12, 465-77

- Mathur, J., (2006) Trichome cell morphogenesis in Arabidopsis: a continuum of cellular decisions. Canadian Journal of Botany. 84, 604-612

- Mehta, S., Hiesinger, P., Beronja, S., Zhai, R., Schulze, K., et al., (2005) Mutations in Drosophila sec15 reveal a function in neuronal targeting for a subset of exocyst components. Neuron. 46, 219-32

- Michaelson, D., Silletti, J., Murphy, G., D'Eustachio, P., Rush, M., Philips, M., (2001) Differential localization of Rho GTPases in live cells: regulation by hypervariable regions and RhoGDI binding. The Journal of Cell Biology.152, 111-26

- Miki, D., Itoh, R., Shimamoto, K., (2005) RNA silencing of single and multiple members in a gene family of rice. Plant Physiology. 138, 1903-1913

- Miya, A., Albert, P., Shinya, T., Desaki, Y., Ichimura, K., Shirasu, K., Narusaka, Y., Kawakami, N., Kaku, H., Shibuya, N., (2007) CERK1, a LysM receptor kinase, is essential for chitin elicitor signaling in Arabidopsis. Proceedings of the National Academy of Sciences USA 104, 19613-19618

- Mizuno, T., (2005) Two-component phosphorelay signal transduction systems in plants: from hormone responses to circadian rhythms. Bioscience Biotechnology and Biochemistry. 69, 2263-2276

- Moeder, W., Yoshioka, K., Klessig, D., (2005) Involvement of the small GTPase Rac in the defense responses of tobacco to pathogens. Molecular Plant Microbe Interactions. 18(2),1624 
- Molendijk, A., Bischoff, F., Rajendrakumar, C., et al. (2001) Arabidopsis thaliana Rop GTPases are localized to tips of root hairs and control polar growth. EMBO Journal. 20, 2779-88

- Molendijk, A., Ruperti, B., Singh, M., et al., (2008) A cysteine-rich receptor like kinase NCRK and a pathogen-induced protein kinase RBK1 are Rop GTPase interactors. Plant Journal. 53, 909-23

- Morel, J., Fromentin, J., Blein, J.,Simon-Plas, F., Elmayan, T., (2004) Rac regulation of NtrbohD, the oxidase responsible for the oxidative burst in elicited tobacco cell. Plant Journal. 37, 282-293

- Murase, K., Shiba, H., Iwano, M., Che, F., Watanabe, M., Isogai, A., Takayama, S., (2004) A membrane-anchored protein kinase involved in Brassica self-incompatibility signaling. Science. 303, 1516-1519

- Muto, H., Yabe, N., Asami, T., Hasunuma, K., Yamamoto, K., (2004) Overexpression of CONSTITUTIVE DIFFERENTIAL GROWTH 1 gene, which encodes a RLCK VIIsubfamily protein kinase, causes abnormal differential and elongation growth after organ differentiation in Arabidopsis. Plant Physiology. 136, 3124-3133

- Nakanomyo, I., Kost, B., Chua, N., Fukuda, H., (2002) Preferential and asymmetrical accumulation of a Rac small GTPase mRNA in differentiating xylem cells of Zinnia elegans. Plant Cell Physiology. 43,1484-1492

- Nakashima, T., Iwashita, T., Fujita, T., Sato, E., Niwano, Y., Kohno, M., Kuwahara, S., Harada, N., Takeshita, S., Oda, T., (2008) A prodigiosin analogue inactivates NADPH oxidase in macrophage cells by inhibiting assembly of p47phox and Rac. The Journal of Biochemistry. 143(1), 107-15

- Nam, K., Li, J., (2002) BRI1/BAK1, a receptor kinase pair mediating brassinosteroid signaling. Cell. 110, 203-212

- Narumiya, S., (1996) The small GTPase Rho: cellular functions and signal transduction. The Journal of Biochemistry. 120, 215-28

- Nibau, C., Wu, H., Cheung, A., (2006) RAC/ROPGTPases: 'hubs' for signal integration and diversification in plants. Trends in Plant Science. 11, 309-315

- Noren, N., Pasquale, E., (2004) Eph receptor-ephrin bidirectional signals that target Ras and Rho proteins. Cellular Signalling. 16, 655-666

- Nystrom, T., Neidhardt, F., (1994) Expression and role of the universal stress protein, UspA, of Escherichia coli during growth arrest. Molecular Microbiology. 11, 537-544

- Ogawa, M., Shinohara, H., Sakagami, Y., Matsubayashi, Y., (2008) Arabidopsis CLV3 peptide directly binds CLV1 ectodomain. Science. 319, 294

- Ono, E., Wong, H., Kawasaki, T., Hasegawa, M., Kodama, O., Shimamoto, K., (2001) Essential role of the small GTPase Rac in disease resistance of rice. Proceedings of the National Academy of Sciences USA. 98, 759-764

- Osborn, T., Pires, C., Birchler, J., et al. (11 co-authors), (2003) Understanding mechanisms of novel gene expression in polyploids. Trends in Genetics. 19, 141-147

- Otvos, K., Pasternak, T., Miskolczi, P., Domoki, M., Dorjgotov, D., Szucs, A., Bottka, S., Dudits, D., Feher, A., (2005) Nitric oxide is required for, and promotes auxin-mediated activation of, cell division and embryogenic cell formation but does not influence cell cycle progression in alfalfa cell cultures. Plant Journal. 43, 849-860

- Oude Weernink, P., Schmidt, M., Jakobs, K., (2004) Regulation and cellular roles of phosphoinositide 5-kinases. European Journal of Pharmacology . 500,87-99

- Paduch, M., Jelen, F., Otlewski, J., (2001) Structure of small G proteins and their regulators. Acta Biochimica Polonica. 48, 829-850 
- Park, J., Choi, H., Lee, S., Lee, T., Yang, Z., Lee, Y., (2000) Rac-related GTP-binding protein in elicitor-induced reactive oxygen generation by suspension-cultured soybean cells. Plant Physiology. 124, 725-32

- Park, J., Gu, Y., Lee, Y., Yang, Z., Lee, Y., (2004) Phosphatidic acid induces leaf cell death in Arabidopsis by activating the Rho-related small G protein GTPase-mediated pathway of reactive oxygen species generation. Plant Physiology. 134, 129-36

- Peck, J., Douglas, G., Wu, C., Burbelo, P., (2002) Human RhoGAP domain containing proteins: structure, function and evolutionary relationships. FEBS Letters. 528, 27-34

- Potikha, T., Collins, C., Johnson, D., Delmer, D., Levine, A., (1999) The involvement of hydrogen peroxide in the differentiation of secondary walls in cotton fibers. Plant Physiology. 119, 849-858

- Potocky, M., Jones, M., Bezvoda, R., Smirnoff, N., Zarsky, V., (2007) Reactive oxygen species produced by NADPH oxidase are involved in pollen tube growth. New Phytologist. 174(4), 742-51

- Qiu, J., Jilk, R., Marks, M., Szymanski, D., (2002) The Arabidopsis SPIKE1 gene is required for normal cell shape control and tissue development. Plant Cell. 14, 101-18

- Rentel, M., Lecourieux, D., Ouaked, F., Usher, S., Petersen, L., Okamoto, H., Knight, H., Peck, S., Grierson, C., Hirt, H., Knight, M., (2004) OXI1 kinase is necessary for oxidative burst-mediated signalling in Arabidopsis. Nature. 427, 858-861

- Rogg, L., Bartel, B., (2001) Auxin signaling: derepression through regulated proteolysis. Developmental Cell. 1, 595-604

- Ros-Barcelo, A., Pomar, F., Lopez-Serrano, M., Martinez, P., Pedreno, M., (2002) Developmental regulation of the $\mathrm{H} 2 \mathrm{O} 2$-producing system and of a basic peroxidase isoenzyme in the Zinnia elegans lignifying xylem. Plant Physiology and Biochemistry. 40, 325-332

- Rossman, K., Der, C., Sondek, J., (2005) GEF means go: turning on RhoGTPases with guanine nucleotide-exchange factors. Nature Reviews Mollecular Cell Biology. 6, 167-180

- Sagi, M., and Fluhr, R., (2006) Production of reactive oxygen species by plant NADPH oxidases. Plant Physiology. 141, 336-340

- Scheffzek, K., Ahmadian, M., (2005) GTPase activating proteins: structural and functional insights 18 years after discovery. Cellular and Molecular Life Science. 62, 3014-3038

- Schiene, K., Pühler, A., Niehaus, K., (2000) Transgenic tobacco plants that express an antisense construct derived from a Medicago sativa cDNA encoding a Rac-related small GTP-binding protein fail to develop necrotic lesions upon elicitor infiltration. Molecular and General Genetics. 263(5), 761-70

- Schiller, M., (2006) Coupling receptor tyrosine kinases to Rho GTPases GEFs what's the link. Cellular Signalling. 18, 1834-43

- Schlessinger, J., (2000) Cell signaling by receptor tyrosine kinases. Cell. 103, 211-225

- Schultheiss, H. Dechert, C., Kogel, K., Hückelhoven, R., (2002) A small GTP-binding host protein is required for entry of powdery mildew fungus into epidermal cells of barley. Plant Physiology. 128(4), 1447-54

- Schultheiss, H., Dechert, C., Kogel, K., Hückelhoven, R., (2003) Functional analysis of barley RAC/ROP G-protein family members in susceptibility to the powdery mildew fungus. Plant Journal. 36(5), 589-601

- Seabra, M., (1998) Membrane association and targeting of prenylated Ras like GTPases. Cellular Signalling.10, 167-72

- Shen, W., Gomez-Cadenas, A., Routly, E., Ho, T., Simmonds, J., Gulick, P., (2001) The salt stress-inducible protein kinase gene, Esi47, from the salt-tolerant wheatgrass Lophopyrum elongatum is involved in plant hormone signaling. Plant Physiology. 125,1429-1441 
- Shiu, S., Bleecker, A., (2001) Receptor-like kinases from Arabidopsis form a monophyletic gene family related to animal receptor kinases. Proceedings of the National Academy of Sciences USA. 98, 10763-10768

- Shiu, S., Bleecker, A., (2003) Expansion of the receptor-like kinase/Pelle gene family and receptor-like proteins in Arabidopsis. Plant Physiology. 132, 530-543

- Shiu, S., Karlowski, W., Pan, R., Tzeng, Y., Mayer, K., Li, W., (2004) Comparative analysis of the receptor-like kinase family in Arabidopsis and rice. Plant Cell. 16, 1220-1234

- Shiu, S., Li, W., (2004) Origins, lineage-specific expansions, and multiple losses of tyrosine kinases in eukaryotes. Molecular Biology and Evolution. 21, 828-840

- Shpak, E., Berthiaume, C., Hill, E., Torii, K., (2004) Synergistic interaction of three ERECTA-family receptor-like kinases controls Arabidopsis organ growth and flower development by promoting cell proliferation. Development. 131, 1491-1501

- Sivaguru, M., Ezaki, B., He, Z., Tong, H., Osawa, H., Baluska, F., Volkmann, D., Matsumoto, H., (2003) Aluminum-induced gene expression and protein localization of a cell wallassociated receptor kinase in Arabidopsis. Plant Physiology. 132, 2256-2266

- Smertenko, A., Jiang, C., Simmons, N., Weeds, A., Davies, D., Hussey, P., (1998) Ser6 in the maize actin depolymerizingfactor, ZmADF3, is phosphorylated by a calcium stimulated protein kinase and is essential for control of functional activity. Plant Journal. 14, 187-193

- Smith, L., Oppenheimer, D., (2005) Spatial control of cell expansion by the plant cytoskeleton. Annual Review of Cell and Developmental Biology. 21, 271-295

- Song, W., Wang, G., Chen, L., Kim, H., Pi, L., Holsten, T., Gardner, J., Wang, B., Zhai, W., Zhu, L., et al., (1995) A receptor kinase-like protein encoded by the rice disease resistance gene Xa21. Science. 270, 1804-1806

- Sorek, N., Poraty, L., Sternberg, H., Bar, E., Lewinsohn, E., Yalovsky, S., (2007) Activation status-coupled transient $\mathrm{S}$ acylation determines membrane partitioning of a plant Rhorelated GTPase. Molecular and Cellular Biology. 27, 2144-2154

- Sormo, C., Leiros, I., Brembu, T., Winge, P., Os, V., Bones, A., (2006) The crystal structure of Arabidopsis thaliana RAC7/ROP9: the first RAS superfamily GTPase from the plant kingdom. Phytochemistry. 67, 2332-2340

- Speranza, A., Scoccianti, V., Crinelli, R., Calzoni, G., Magnani M., (2001) Inhibition of Proteasome Activity Strongly Affects Kiwifruit Pollen Germination. Involvement of the Ubiquitin/Proteasome Pathway as a Major Regulator Plant Physiology. 126(3), 1150 - 1161

- Sprang, S., (1997) G proteins, effectors and GAPs: structure and mechanism. Current Opinion in Structural Biology. 7(6), 849-56

- Stone, J., Walker, J., (1995) Plant protein kinase families and signal transduction. Plant Physiology. 108, 451-457

- Stracke, S., Kistner, C., Yoshida, S., Mulder, L., Sato, S., Kaneko, T., Tabata, S., Sandal, N., Stougaard, J., Szczyglowski, K., et al., (2002) A plant receptor like kinase required for both bacterial and fungal symbiosis. Nature. 417, 959-962

- Swiderski, M., Innes, R., (2001) The Arabidopsis PBS1 resistance gene encodes a member of a novel protein kinase subfamily. Plant Journal. 26, 101-112

- Szücs, A., Dorjgotov, D., Ötvös, K., Fodor, C., Domoki, M., Györgyey, J., Kaló, P., Kiss, G.B., Dudits, D., Fehér, A., (2006) Characterization of three Rop GTPase genes of alfalfa (Medicago sativa L.). Biochimica Biophysica Acta. 1759, 108-15

- Szymanski, D., (2005) Breaking the WAVE complex: the point of Arabidopsis trichomes. Current Opinion in Plant Biology. 8(1),103-12

- Takai, Y., Sasaki, T., Matozaki, T., (2001) Small GTP-binding proteins. Physiological Reviews. 81, 153-208 
- Takeda, S., Gapper, C., Kaya, H., Bell,E., Kuchitsu, K., Dolan, L., (2008) Local positive feedback regulation determines cell shape in root hair cells. Science. 319, 1241

- Tang, X., Frederick, R., Zhou, J., Halterman, D., Jia, Y., Martin, G., (1996) Initiation of plant disease resistance by physical interaction of AvrPto and Pto kinase. Science. 274, 20602063

- Tang, W., Kim, T., Oses-Prieto, J., Sun, Y., Deng, Z., Zhu, S.,Wang, R., Burlingame, A., Wang, Z., (2008) BSKs mediate signal transduction from the receptor kinase BRI1 in Arabidopsis. Science. 321, 557-560.

- Tao, L., Cheung, A., Wu, H., (2002) Plant Rac-like GTPases are activated by auxin and mediate auxin-responsive gene expression. Plant Cell .14, 2745-60

- Tao, L., Cheung, Y., Nibau, C., Wu, H., (2005) RAC GTPases in Tobacco and Arabidopsis Mediate Auxin-Induced Formation of Proteolytically Active Nuclear Protein Bodies That Contain AUX/IAA Proteins. The Plant Cell. 17, 2369-2383

- Thao, N., Chen, L., Nakashima, A., et al. (2007) RAR1 and HSP90 form a complex with Rac/Rop GTPase and function in innate-immune responses in rice. Plant Cell. 19, 4035-45

- Thapar, R., Karnoub, A., Campbell, S., (2002) Structural and biophysical insights into the role of the insert region in Rac1 function. Biochemistry. 41, 3875-3883

- Thomas, C., Fricke, I., Scrima, A., Berken, A., Wittinghofer, A., (2007) Structural evidence for a common intermediate in small G protein-GEF reactions. Molecular Cell. 25, 141-149

- Thompson, J., Higgins, D., Gibson, T., (1994) Clustal W: improving the sensitivity of progressive multiple sequence alignment through sequence weighting, position-specific gap penalties and weight matrix choice. Nucleic Acids Research. 22, 4673-4680

- Torii, K., Mitsukawa, N., Oosumi, T., Matsuura, Y., Yokoyama, R., Whittier, R., Komeda, Y., (1996) The Arabidopsis ERECTA gene encodes a putative receptor protein kinase with extracellular leucine-rich repeats. Plant Cell. 8, 735-746

- Torres, M., Dangl, J., (2005) Functions of the respiratory burst oxidase in biotic interactions, abiotic stress and development. Current Opinion in Plant Biology. 8, 397-403

- Torres, M., Dangl, J., Jones, J., (2002) Arabidopsis gp91phox homologues AtrbohD and AtrbohF are required for accumulation of reactive oxygen intermediates in the plant defense response. Proceedings of the National Academy of Sciences USA. 99, 517-522

- Trotochaud, A., Hao, T., Wu, G., Yang, Z., Clark, S., (1999) The CLAVATA1 receptor-like kinase requires CLAVATA3 for its assembly into a signaling complex that includes KAPP and a Rho-related protein. Plant Cell. 11, 393-406

- Tu, H., Wigler, M., (1999) Genetic evidence for Pak1 autoinhibition and its release by Cdc42. Molecular and Cellular Biology. 19, 602-611

- Uhrig, J., Mutondo, M., Zimmermann, I., et al, (2007) The role of Arabidopsis SCAR genes in ARP2-ARP3-dependent cell morphogenesis. Development. 134(5), 967-77

- Ullrich, A., Schlessing, J., (1990) Signal transduction by receptors with tyrosine kinase activity. Cell. 61, 203-212

- Urao, T., Yamaguchi-Shinozaki, K., Shinozaki, K., (2000) Two-component systems in plant signal transduction. Trends in Plant Science. 5, 67- 74

- Van Aelst, L., Cline, H., (2004) Rho GTPases and activity-dependent dendrite development. Current Opinion in Neurobiology. 14, 297-304

- Vandesompele, J., De Preter, K., Pattyn, F., Poppe, B., Van Roy, N., De Paepe, A., Speleman, F., (2002) Accurate normalization of real-time quantitative RT-PCR data by geometric averaging of multiple internal control genes. Genome Biology. 3,1465-6906

- Vernoud, V., Horton, AC., Yang, Z., Nielsen, E., (2003) Analysis of the small GTPase gene superfamily in Arabidopsis. Plant Physiology. 131, 1191-1208 
- Veronese, P., Nakagami, H., Bluhm, B., Abuqamar, S., Chen, X., Salmeron, J., Dietrich, R., Hirt, H., Mengiste, T., (2006) The Membrane-Anchored BOTRYTIS-INDUCED KINASE1 Plays Distinct Roles in Arabidopsis Resistance to Necrotrophic and Biotrophic Pathogens. Plant Cell. 18, 257-273

- Vetter, IR., Wittinghofer, A., (2001) The guanine nucleotide-binding switch in three dimensions. Science. 294, 1299-1304

- Vision, T., Brown, D., Tanksley, S., (2000) The origins of genomic duplications in Arabidopsis. Science. 290, 2114-2117

- Walker, J., (1994) Structure and function of the receptor-like protein kinases of higher plants. Plant Molecular Biology. 26, 1599-1609

- Walker, J., Zhang, R., (1990) Relationship of a putative receptor protein kinase from maize to the S-locus glycoproteins of Brassica. Nature. 345, 743-746

- Walker, S., Wu, W., Cerione, R., Brown, H., (2000) Activation of phospholipase D1 by Cdc42 requires the Rho insert region. Journal of Biological Chemistry. 275, 15665-15668

- Walther, D., Brunnemann, R., Selbig, J., (2007) The regulatory code for transcriptional response diversity and its relation to genome structural properties in Arabidopsis thaliana. PLoS Genetics. 3, 11

- Wang, X., Goeshe, B., Soderblom, E., Phinney, B., Kuchar, B.,et al., (2005) Identification and functional analysis of in vivo phosphorylation sites of the Arabidopsis BRASSINOSTEROID-INSENSITIVE1 receptor kinase. Plant Cell. 17, 1685-1703

- Wang, X., Li, X., Meisenhelder, J., Hunter, T., Yoshida, S., et al., (2005) Autoregulation and homodimerization are involved in the activation of the plant steroid receptor BRI1. Developmental Cell. 8, 855-865

- Wennerberg, K., Der, C., (2004) Rho-family GTPases: it's not only Rac and Rho (and I like it). J. Cell Sci. 117, 1301-1312.

- Wennerberg, K., Rossman, K., Der, C., (2005) The Ras superfamily at a glance. Journal of Cell Science. 118, 843-6

- Winge, P., Brembu, T., Bones, A., (1997) Cloning and characterization of Rac-like cDNAs from Arabidopsis thaliana. Plant Molecular Biology. 35, 483-95

- Winge, P., Brembu, T., Kristensen, R., Bones, A., (2000) Genetic structure and evolution of RAC-GTPases in Arabidopsis thaliana. Genetics 156, 1959-1971

- Wisniewska, J., Xu, J., Seifertova, D., Brewer, P., Ruzicka, K., et al., (2006) Polar PIN localization directs auxin flow in plants. Science. 312, 883

- Wong, H., Pinontoan. R., Hayashi, K., et al., (2007) Regulation of rice NADPH oxidase by binding of Rac GTPase to its N-terminal extension. Plant Cell .19, 4022-34

- Wong, H., Sakamoto, T., Kawasaki, T., Umemura, K., Shimamoto, K., (2004) Downregulation of metallothionein, a reactive oxygen scavenger, by the small GTPase OsRac1 in rice. Plant Physiology. 135, 1447-1456

- Wu, G., Gu, Y., Li, S., Yang, Z., (2001) A genome-wide analysis of Arabidopsis Ropinteractive CRIB motif-containing proteins that act as Rop GTPase targets. Plant Cell. 13, 2841-56

- Wu, G., Li, H., Yang, Z., (2000) Arabidopsis RopGAPs are a novel family of Rho GTPaseactivating proteins that require the Cdc42/Racinteractive binding motif for Rop-specific GTPase stimulation. Plant Physiology. 124, 1625-36

- Xin, Z., Zhao, Y., Zheng, Z., (2005) Transcriptome analysis reveals specific modulation of abscisic acid signaling by ROP10 small GTPase in Arabidopsis. Plant Physiology. 139, $1350-1365$ 
- Yamaguchi, Y., Pearce, G., Ryan, C., (2006) The cell surface leucine-rich repeat receptor for AtPep1, an endogenous peptide elicitor inArabidopsis, is functional in transgenic tobacco cells. Proceedings of the National Academy of Sciences USA. 103, 10104-10109

- Yamaguchi-Shinozaki, K., Shinozaki, K., (1993) Characterization of the expression of a desiccation-responsive $\mathrm{rd} 29$ gene of Arabidopsis thaliana and analysis of its promoter in transgenic plants. Molecular and General Genetics. 236, 331-340

- Yang, Z. and Fu, Y., (2007) ROP/RAC GTPase signaling. Current Opinion in Plant Biology. $10,490-4$

- Yang, Z., (2002) Small GTPases: versatile signaling switches in plants. Plant Cell. 14, S375S388.

- Yoshioka, H., Numata, N., Nakajima, K., Katou, S., Kawakita, K., Rowland, O., Jones, J.D., and Doke, N., (2003) Nicotiana benthamiana gp91phox homologs NbrbohA and NbrbohB participate in $\mathrm{H}_{2} \mathrm{O}_{2}$ accumulation and resistance to Phytophthora infestans. Plant Cell. 15, 706-718

- Yoshioka, H., Sugie, K., Park, H.J., Maeda, H., Tsuda, N., Kawakita, K., and Doke, N., (2001) Induction of plant gp91 phox homolog by fungal cell wall, arachidonic acid, and salicylic acid in potato. Molecular Plant Microbe Interaction. 14, 725-736

- Young, S., Ambroziak, P., Kim, E., Clarke, S., (2000) Postprenylation protein processing: CXXX (CaaX) endoproteases and isoprenylcyteine carboxyl methyltransferase. In $\mathrm{F}$ Tamanoi, DS Sigman, eds, Protein Lipidation, Ed 2, Vol 21. Academic Press, San Diego, $156-213$

- Zajac, A., Sun, X., Zhang, J., Guo, W., (2005) Cyclical regulation of the exocyst and cell polarity determinants for polarized cell growth. Molecular Biology of the Cell. 16, 1500-12

- Zarembinski, T., Hung, L., Mueller-Dieckmann, H., Kim, K., Yokota, H., Kim, R., Kim., (1998) Structure-based assignment of the biochemical function of a hypothetical protein: a test case of structural genomics. Proceedings of the National Academy of Sciences USA. 95,15189-15193

- Zhang, Y., McCormick, S., (2007) A distinct mechanism regulating a pollen-specific guanine nucleotide exchange factor for the small GTPase Rop in Arabidopsis thaliana. Proceedings of the National Academy of Sciences USA. 20;104(47):18830-5

- Zhao, Z., Manser, E., (2005) PAK and other Rho-associated kinases effectors with surprisingly diverse mechanisms of regulation. Biochemical Journal. 386, 201-14

- Zheng, Z, Yang, Z., (2000) The Rop GTPase: an emerging signaling switch in plants. Plant Molecular Biology. 44, 1-9

- Zheng, Z., Nafisi, M., Tam, A., Li, H., Crowell, D., Chary, N., Schroeder, J., Shen, J., Yang, Z., (2002) Plasma Membrane-Associated ROP10 Small GTPase Is a Specific Negative Regulator of Abscisic Acid Responses in Arabidopsis. Plant Cell. 14, 2787-2797

- Zheng, Z., Yang, Z., (2000) The ROP GTPase switch turns on polar growth in pollen. Plant Science. 5, 298-303

- Zhou, A., Wang, H., Walker, J., Li, J., (2004) BRL1, a leucine-rich repeat receptor-like protein kinase, is functionally redundant with BRI1 in regulating Arabidopsis brassinosteroid signaling. Plant Journal. 40, 399-409

- Zhou, J., Loh, Y., Bressan, R., Martin, G., (1995) The tomato gene Ptil encodes a serine/threonine kinase that is phosphorylated by Pto and is involved in the hypersensitive response. Cell. 83, 925-935

- Zimmermann, P., Hennig, L., Gruissem, W., (2005) Gene-expression analysis and network discovery using Genevestigator. Trends in Plant Science. 10,407-409

- Zimmermann, P., Hirsch-Hoffmann, M., Hennig, L., Gruissem, W., (2004) Genevestigator. Arabidopsis microarray database and analysis toolbox. Plant Physiology.136, 2621-32 
- Zipfel, C., Kunze, G., Chinchilla, D., Caniard, A., Jones, J., Boller, T., Felix, G., (2006) Perception of the bacterial PAMP EF-Tu by the receptor EFR restricts Agrobacteriummediated transformation. Cell. 125, 749-760

- Zong, H., Kaibuchi, K., Quilliam, L., (2001) The insert region of RhoA is essential for Rho kinase activation and cellular transformation. Molecular and Cellular Biology. 21, 52875298

- Zuo, J., Niu, Q., Chua, N., (2000). An estrogen receptor-based transactivator XVE mediates highly inducible gene expression in transgenic plants. Plant Journal. 24, 265-273 


\section{AKNOWLEDGMENTS}

I would first like to thank my supervisor Dr. Attila Fehér for his guidance and all of the opportunities he has given to me over the years. I have been able to grow professionally, intellectually, and personally while working in his laboratory. I am truly grateful for all of his mentorship and guidance.

I am also grateful to Dr. Zoltan Magyar for his valuable ideas, comments, and suggestions on writing the thesis.

Many thanks to all of my colleagues from Biological Research Center, Laboratory of Functional Cell Biology: Ötvös Krisztina, Domoki Mónika, Biró Judit, Dunainé Fodor Csilla, Szücs Attila, Dulguun Dorjgotov, Katona Gyöngyvér and Nagy Róza, for their strong support and help.

Finally, I am thankful to my family and my friends from Szeged for their valuable help and friendship that I will never forget. 